\title{
ANALYSIS OF THE ASYMMETRIC SHORTEST QUEUE PROBLEM
}

\author{
I.J.B.F. ADAN, J. WESSELS and W.H.M. ZIJM * \\ Department of Mathematics and Computing Science, Eindhoven University of Technology, \\ P.O. Box 513, 5600-MB Eindhoven, The Netherlands
}

Received 18 May 1990; revised 29 October 1990

\begin{abstract}
In this paper we study a system consisting of two parallel servers with different service rates. Jobs arrive according to a Poisson stream and generate an exponentially distributed workload. On arrival a job joins the shortest queue and in case both queues have equal lengths, he joins the first queue with probability $q$ and the second one with probability $1-q$, where $q$ is an arbitrary number between 0 and 1 . In a previous paper we showed for the symmetric problem, that is for equal service rates and $q=1 / 2$, that the equilibrium distribution of the lengths of the two queues can be exactly represented by an infinite sum of product form solutions by using an elementary compensation procedure. The main purpose of the present paper is to prove a similar product form result for the asymmetric problem by using a generalization of the compensation procedure. Furthermore, it is shown that the product form representation leads to a numerically efficient algorithm. Essentially, the method exploits the convergence properties of the series of product forms. Because of the fast convergence an efficient method is obtained with upper and lower bounds for the exact solution. For states further away from the origin the convergence is faster. This aspect is also exploited in the paper.
\end{abstract}

Keywords: Bounds, difference equation, product form, queues in parallel, stationary queue length distribution, shortest queue problem.

\section{Introduction}

Consider a queueing system consisting of two parallel servers with different service rates. Jobs arrive according to a Poisson stream and generate an exponentially distributed workload. On arrival a job joins the shortest queue and in case both queues have equal lengths, he joins the first queue with probability $1-q$ and the second one with probability $q$, where $q$ is an arbitrary number between 0 and 1 . This problem is known as the asymmetric shortest queue problem, that is, the asymmetric variant of the symmetric problem with identical servers and routing probability $q=1 / 2$. Haight [18] originally introduced the problem.

* University of Twente, Department of Mechanical Engineering, P.O. Box 217, 7500 AE Enschede, The Netherlands.

(C) J.C. Baltzer A.G. Scientific Publishing Company 
Kingman [22] and Flatto and McKean [10] treated the symmetric problem by using a generating function analysis. They showed that the generating function for the equilibrium distribution of the lengths of the two queues is a meromorphic function. Then by the decomposition of the generating function into partial fractions, it follows that the equilibrium probabilities can be represented by an infinite sum of product form solutions. However, the decomposition leads to cumbersome formulae for the equilibrium probabilities and the method does not seem to be generalizable to the asymmetric problem. Cohen and Boxma [7] and Fayolle and Iasnogorodski $[9,21]$ studied the asymmetric shortest queue problem and showed that the analysis of the asymmetric shortest queue problem can be reduced to a simultaneous boundary value problem in two unknowns. The resulting boundary value problem however, is not of a standard type and further research remains to be done here. Knessl, Matkowsky, Schuss and Tier [23] obtained asymptotic expressions of the stationary queue length distribution for the asymmetric shortest queue problem. So far, these are the only analytic results for the asymmetric problem available in the literature.

In a previous paper [4] we showed for the symmetric shortest queue problem that the equilibrium distribution of the lengths of the two queues can be found in an elementary way directly from the equilibrium equations. The key idea is a compensation procedure: the queue length distribution can be represented by an infinite sum of product form solutions, which is generated term by term, such that each term compensates for the error, introduced by its preceding term, on one of the boundaries of the state space. In [3] we showed that this compensation procedure can be easily extended to the "simple" asymmetric shortest queue problem. By "simple" we mean a system with identical servers and routing probability $q \neq 1 / 2$. The purpose of the present paper is to generalize the compensation procedure to the "hard" asymmetric problem in which the service rates are different as well. This generalization is not straightforward. For the symmetric problem we had to construct a solution of the equilibrium equations on essentially one half of the state space. For the asymmetric problem we distinguish between two different, but coupled regions and the basic problem for the construction of the solutions on both regions, is how to compensate for the errors introduced by both solutions on the common boundary of the two regions. Only for the simple asymmetric case this problem can be solved easily, since in that case the equilibrium equations in the interior of these two regions are still a mirror image of each other. Due to the coupling between the two regions, Cohen and Boxma [7] obtain in their study a simultaneous boundary value problem.

The main resuit is, also for the hard asymmetric problem, that the equilibrium distribution of the lengths of both queues can in each region be exactly represented by an infinite sum of product form solutions. Because of the interaction of the solutions on both regions, this sum does not have a linear structure as in the symmetric case, but a binary tree structure. The compensation procedure yields recursion relations for the successive terms in the infinite sum. The terms 
decrease exponentially fast. Typically is that on one hand the analysis is rather complicated, whereas on the other hand the final results are simple and can be easily used for numerical computations. Also, the product form expressions for the equilibrium probabilities easily lead to similar expressions for the moments of the sojourn time. The compensation approach bears flexibility towards small modifications in the model. It is shown that the compensation approach can be extended to the threshold shortest queue problem and to the shortest queue problem for parallel multi server queues.

Most numerical studies of the shortest queue problem deal with the evaluation of approximating models, constructed by truncating appropriate state variables. On the symmetric problem, we mention that Gertsbakh [14] treated a modified shortest queue problem by limiting the difference between the lengths of both queues and obtained a numerical solution by using the matrix-geometric approach developed by Neuts [24]. Conolly [8] discussed the finite waitingroom version of the shortest queue problem and showed that this problem can be solved efficiently, essentially by dimension reduction (see also section 18). Halfin [19] obtained bounds for the equilibrium distribution of the lengths of the two queues by applying linear programming techniques. Foschini and Salz [11] obtained heavy traffic approximations for the queue length distribution. In [4] it appeared that the exact product form representation for the queue length distribution offers an efficient computational procedure for the symmetric problem. Regarding the asymmetric problem, Grassmann [15] performed a numerical study of the steady state as well as the transient behaviour of the asymmetric system, but to restrict the state space he treated both queues as bounded. Treating one queue as bounded, Rao and Posner [25] showed that the equilibrium distribution can be expressed in a modified matrix-geometric form and they developed an efficient computational procedure. Hooghiemstra, Keane and Van de Ree [20] proposed a power series method to calculate the stationary queue length distribution for fairly general multidimensional exponential queueing systems. Although the power series method works numerically satisfactory for the shortest queue problem, see e.g. Blanc [5,6], the theoretical foundation of this method is still incomplete. Finally, a common disadvantage of the numerical methods mentioned is that in general no error bounds can be given.

In this paper it is shown that the product form representation offers efficient and accurate numerical algorithms. Due to the recursion relations, the successive terms of the binary tree of product forms can be easily calculated and, because of the exponential convergence, few terms often suffice to obtain an accurate approximation. In addition, we provide bounds for the error of each partial tree. The bounds are obtained by bounding the subtrees below the leaves of the partial tree by positive geometrical trees. The contribution of each geometrical tree can be easily calculated. Based on these properties, an efficient numerical algorithm with tight bounds on the error of each partial tree is obtained. These algorithms apply to the exact model and basically consist of computing a sequence of partial trees 
until the desired accuracy is reached. In each cycle we have to decide for which leaf of the current partial tree the immediate successors are computed. Two strategies are considered. The first strategy computes in each cycle the immediate successors of all leaves of the partial tree. The other one exploits the relative importance of the branches of the tree by computing only the immediate successors of the leaf the subtree of which has maximum weight. For highly unbalanced systems however, the series of product forms do not converge absolutely in a usually small region near the origin of the state space. A system is called highly unbalanced if one of the servers is working much faster than the other one. Therefore we propose a numerically stable recursive algorithm for solving the equilibrium equations in a bounded region near the origin of the state space. This approach can also be used if convergence of the series of product forms in states around the origin is slow compared to the convergence in states further away from the origin.

This paper is organized as follows. In section 2 we present the equilibrium equations. In section 3 we analyze the asymptotic behaviour of the equilibrium probabilities, essentially based on numerical experience. The subsequent three sections are devoted to the compensation method. In particular, section 6 presents the formal definition of the compensation method. Sections 7,8 and 9 are devoted to prove that the series of product forms, which are formally defined in section 6 , converge absolutely. In section 10 the asymptotic behaviour of the series of product forms is analyzed. Section 11 presents the main result, stating that the equilibrium probabilities can be represented by an infinite sum of product form solutions. In sections 12 and 13 series of product forms are derived for the normalizing constant and the moments of the sojourn time. Sections 14 and 15 discuss the extension of the compensation approach to the threshold shortest queue problem and to the shortest queue problem for parallel multi server queues, and thereby conclude the analytical treatment. The rest of the paper approaches the product form representation from a computational point of view. In section 16 we derive bounds for the contribution of each subtree and the next section presents the basic scheme for the computation of a product form tree. In section 18 we propose an efficient and numerically stable algorithm for solving the equilibrium equations in a bounded region. Section 19 discusses the computation scheme, which combines the advantages of the product form representation and the efficient scheme in section 18, and presents numerical results. An alternative strategy for the computation of product form trees is discussed in section 20 . The final section is devoted to conclusions. The appendix includes the proof of the bounds.

\section{Equilibrium equations}

For simplicity of notation the servers have service rates $\gamma_{1}$ and $\gamma_{2}$ respectively with $\gamma_{1}>0, \gamma_{2}>0$ and $\gamma_{1}+\gamma_{2}=2$, the Poisson arrival process has a rate $2 \rho$ with 


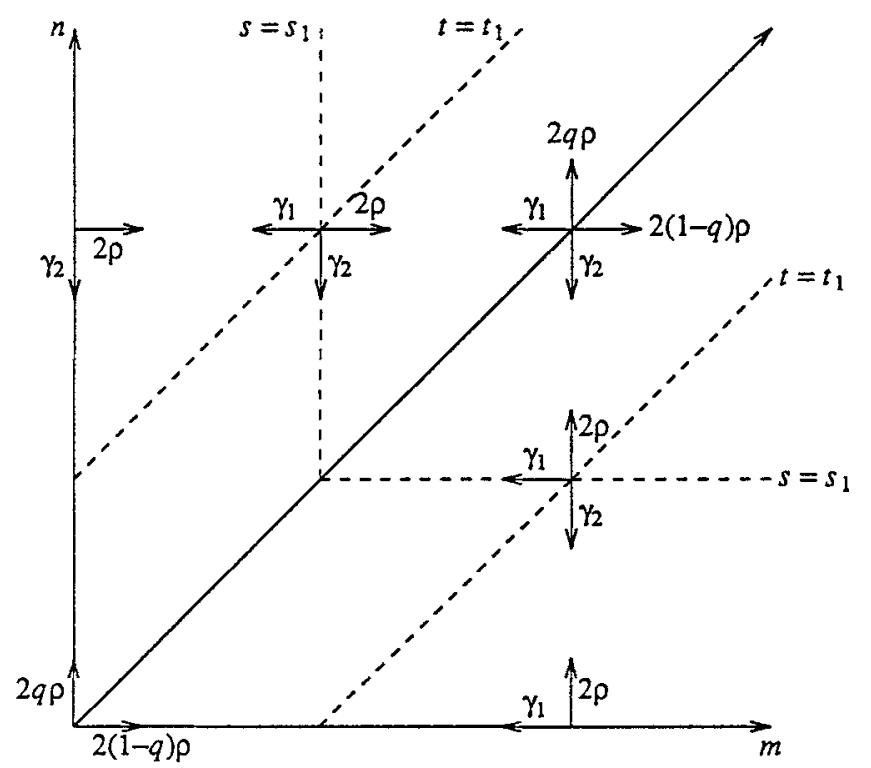

Fig. 1. Transition rate diagram.

$0<\rho<1$ and on arrival each job generates an exponentially distributed workload with unit mean. $q$ is the probability that an arriving job is sent to the second queue in case both queues have equal lengths. This parallel queue system can be represented by a continuous time Markov process, with a state space $\mathscr{S}$ consisting of the pairs $(m, n), m, n=0,1, \ldots$ where $m$ and $n$ are the lengths of the two queues. Let $\left\{p_{m, n}\right\}$ be the equilibrium distribution of the lengths of the two queues. For the symmetric problem, that is $\gamma_{1}=\gamma_{2}=1$ and $q=1 / 2$, we have $p_{m, n}=p_{n, m}$. Hence, for this special case the analysis can be restricted to the upper triangle $m \leqslant n$ and in [4] we proved that the equilibrium probabilities $p_{m, n}$ can be represented by an infinite sum of product form solutions. For the asymmetric problem we cannot restrict the analysis to the upper triangle, but we have to construct solutions on the upper triangle $m \leqslant n$ as well as on the lower triangle $m \geqslant n$. The difficulty arises from the fact that both solutions interact on the diagonal. The first purpose of this paper is to prove that these solutions can be represented by a series of product forms. For the purpose of analysis we prefer to have the coordinate axes along the boundaries of the upper and lower triangle. Therefore, we work with the coordinates $s=m$ and $t=n-m$ in the upper triangle, and with the coordinates $s=n$ and $t=m-n$ in the lower triangle. In fig. 1 we display the transition rate diagram. Set for all $s \geqslant 0$ and $t \geqslant 0$,

$$
Q_{s, t}=p_{s, s+t}, \quad q_{s, t}=p_{s+t, s} .
$$

Then by definition $Q_{s, 0}=q_{s, 0}$. In the sequel we use upper case letters for solutions in the upper triangle and lower case letters for solutions in the lower triangle. The 
equilibrium equations in the upper triangle state that

$$
\begin{aligned}
& Q_{s, t} 2(\rho+1)=Q_{s-1, t+1} 2 \rho+Q_{s, t+1} \gamma_{2}+Q_{s+1, t-1} \gamma_{1} \\
& \quad \text { if } s>0, t>1, \\
& Q_{s, 1} 2(\rho+1)=Q_{s-1,2} 2 \rho+Q_{s, 2} \gamma_{2}+Q_{s+1,0} \gamma_{1}+Q_{s, 0} 2 q \rho \\
& \quad \text { if } s>0, \\
& Q_{0, t}\left(2 \rho+\gamma_{2}\right)=Q_{0, t+1} \gamma_{2}+Q_{1, t-1} \gamma_{1} \quad \text { if } t>1, \\
& Q_{0,1}\left(2 \rho+\gamma_{2}\right)=Q_{0,2} \gamma_{2}+Q_{1,0} \gamma_{1}+Q_{0,0} 2 q \rho ;
\end{aligned}
$$

and in the lower triangle

$$
\begin{aligned}
& q_{s, t} 2(\rho+1)=q_{s-1, t+1} 2 \rho+q_{s, t+1} \gamma_{1}+q_{s+1, t-1} \gamma_{2} \quad \text { if } s>0, t>1, \\
& q_{s, 1} 2(\rho+1)=q_{s-1,2} 2 \rho+q_{s, 2} \gamma_{1}+q_{s+1,0} \gamma_{2}+q_{s, 0} 2(1-q) \rho \\
& \quad \text { if } s>0, \\
& q_{0, t}\left(2 \rho+\gamma_{1}\right)=q_{0, t+1} \gamma_{1}+q_{1, t-1} \gamma_{2} \quad \text { if } t>1, \\
& q_{0,1}\left(2 \rho+\gamma_{1}\right)=q_{0,2} \gamma_{1}+q_{1,0} \gamma_{2}+q_{0,0} 2(1-q) \rho .
\end{aligned}
$$

The equations on the diagonal, i.e. on the line $t=0$ state that

$$
\begin{aligned}
& Q_{s, 0} 2(\rho+1)=Q_{s-1,1} 2 \rho+Q_{s, 1} \gamma_{2}+q_{s-1,1} 2 \rho+q_{s, 1} \gamma_{1} \text { if } s>0, \\
& Q_{0,0} 2 \rho=Q_{0,1} \gamma_{2}+q_{0,1} \gamma_{1} .
\end{aligned}
$$

In the following sections we will prove that there exist parameters $\alpha_{i}, \eta_{i}$ and $\xi_{i}$ and coefficients $c_{i}, d_{i}$ and $e_{i}$ such that, for all $s \geqslant 0$ and $t \geqslant 1$,

$$
Q_{s, t}=\sum_{i=0}^{\infty} c_{i} \alpha_{i}^{s} \eta_{i}^{t}, \quad q_{s, t}=\sum_{i=0}^{\infty} d_{i} \alpha_{i}^{s} \xi_{i}^{t},
$$

and, for all $s \geqslant 0$,

$$
Q_{s, 0}=q_{s, 0}=\sum_{i=0}^{\infty} e_{i} \alpha_{i}^{s} .
$$

\section{The initial asymptotic solution}

The objective in this section is to study the structure of the equilibrium probabilities. In particular we investigate whether the equilibrium probabilities have some kind of separable structure. Obviously, the equilibrium equations do not allow a separable solution of the form $Q_{s, t}=\alpha^{s} \beta^{t}$ and $q_{s, t}=\alpha^{s} \delta^{t}$. However, numerical experience suggests that there exist parameters $\alpha, \beta$ and $\delta$ such that, as $s \rightarrow \infty$ and $t \geqslant 1$,

$$
Q_{s, t} \sim K \alpha^{s} \beta^{t}, \quad q_{s, t} \sim L \alpha^{s} \delta^{t},
$$

and, as $s \rightarrow \infty$ and $t=0$,

$$
Q_{s, 0}=q_{s, 0} \sim M \alpha^{s}
$$


for some constants $K, L$ and $M$. Below we derive explicit expressions for the parameters $\alpha, \beta$ and $\delta$ and the constants $K, L$ and $M$. The parameter $\alpha$ follows from a balance argument. Denote by $A_{k}$ the set of states for which there are $k$ jobs in the system and let $A=A_{k+1}+A_{k+2}+\ldots$. Then we apply the balance principle,

Rate out of the set of states $A=$ Rate into the set of states $A$.

Notice that it is only possible to leave set $A$ via a state in $A_{k+1}$ (with rate $\gamma_{1}+\gamma_{2}=2$, except for those states for which one queue is empty). So the rate out of the set of states $A$ equals $P\left(A_{k+1}\right) 2-Q_{0, k+1} \gamma_{1}-q_{0, k+1} \gamma_{2}$. Further it is only possible to enter set $A$ via a state in $A_{k}$ (with rate $2 \rho$ ). So the rate into set $A$ equals $P\left(A_{k}\right) 2 \rho$. Equating the rate out of and the rate into set $A$, yields for all $k \geqslant 0$ that

$$
P\left(A_{k+1}\right) 2-Q_{0, k+1} \gamma_{2}-q_{0, k+1} \gamma_{1}=P\left(A_{k}\right) 2 \rho .
$$

Combining that equality for $k=2 N$ and $k=2 N+1$ and neglecting the contribution of $Q_{0, k+1}$ and $q_{0, k+1}$ yields that

$$
P\left(A_{2 N+2}\right) \approx P\left(A_{2 N}\right) \rho^{2}
$$

valid for large $N$. On the other hand, by the asymptotic solutions (11), we have for large $N$ that

$$
P\left(A_{2 N}\right) \approx C \alpha^{N}
$$

for some constant $C$, which is independent of $N$. Then together with (12) it is suggested that

$$
\alpha=\rho^{2} \text {. }
$$

The solutions (11) describe the behaviour of the equilibrium probabilities away from the boundary $s=0$. Hence, the solutions (11) have to satisfy the equilibrium equations in that region, that is, eqs. (1), (2), (5), (6) and (9). Based on these equations, we derive expressions for the parameters $\beta$ and $\delta$ and the constants $K$, $L$ and $M$. First, the parameters $\beta$ and $\delta$ follow by observing that the product $\alpha^{s} \beta^{t}$ has to satisfy eq. (1) and $\alpha^{s} \delta^{t}$ has to satisfy eq. (5). Inserting $\alpha^{s} \beta^{t}$ into eq. (1) and dividing both sides by the common factor $\alpha^{s-1} \beta^{t-1}$ yields a quadratic equation for $\beta$. Accordingly we obtain a quadratic equation for $\delta$. This is stated in the following lemma.

\section{LEMMA 1}

(i) The product $\alpha^{s} \beta^{t}$ is a solution of eq. (1) if and only if $\alpha$ and $\beta$ satisfy the quadratic equation

$$
\alpha \beta 2(\rho+1)=\beta^{2} 2 \rho+\alpha \beta^{2} \gamma_{2}+\alpha^{2} \gamma_{1} .
$$

(ii) The product $\alpha^{s} \delta^{t}$ is a solution of eq. (5) if and only if $\alpha$ and $\delta$ satisfy the quadratic equation

$$
\alpha \delta 2(\rho+1)=\delta^{2} 2 \rho+\alpha \delta^{2} \gamma_{1}+\alpha^{2} \gamma_{2}
$$


For fixed $\alpha$ the quadratic eq. (13) in $\beta$ is solved by

$$
X_{ \pm}(\alpha)=\alpha \frac{\rho+1 \pm \sqrt{(\rho+1)^{2}-\left(2 \rho+\alpha \gamma_{2}\right) \gamma_{1}}}{2 \rho+\alpha \gamma_{2}}
$$

and for fixed $\beta$ the equation in $\alpha$ is solved by

$$
Y_{ \pm}(\beta)=\beta \frac{2(\rho+1)-\beta \gamma_{2} \pm \sqrt{\left(2(\rho+1)-\beta \gamma_{2}\right)^{2}-8 \rho \gamma_{1}}}{2 \gamma_{1}} .
$$

Analogously $x_{ \pm}(\alpha)$ are defined as the roots of (14) for fixed $\alpha$ and $y_{+}(\delta)$ as the roots of (14) for fixed $\delta$. By lemma 1(i) we obtain two roots $\beta=X_{+}\left(\rho^{2}\right)=\rho$ and $\beta=X_{-}\left(\rho^{2}\right)=\rho^{2} \gamma_{1} /\left(2+\rho \gamma_{2}\right)$ for fixed $\alpha=\rho^{2}$. The root $\beta=\rho$ yields the asymptotic solution $Q_{s, t} \sim K \rho^{2 s} \rho^{t}$ for some $K$, which corresponds to the equilibrium distribution of two independent $M / M / 1$ queues, each with a workload $\rho$. It is very unlikely that the equilibrium distribution of the shortest queue problem behaves asymptotically like this distribution. Therefore, the only reasonable choice is the smaller root $\beta=\rho^{2} \gamma_{1} /\left(2+\rho \gamma_{2}\right)$. Accordingly we obtain that $\delta=$ $x_{-}\left(\rho^{2}\right)=\rho^{2} \gamma_{2} /\left(2+\rho \gamma_{1}\right)$. A relation between the constants $K$ and $M$ is found by inserting the asymptotic solutions (11) into eq. (2). That leads to the equation

$$
K \alpha^{s} \beta 2(\rho+1)=K \alpha^{s-1} \beta^{2}\left(2 \rho+\alpha \gamma_{2}\right)+M \alpha^{s}\left(\alpha \gamma_{1}+2 q \rho\right) \text {. }
$$

Dividing both sides by $\alpha^{s-1}$ and then inserting eq. (13) yields

$$
K \alpha \gamma_{1}=M\left(\alpha \gamma_{1}+2 q \rho\right),
$$

which is the desired relation between $K$ and $M$. Accordingly, by inserting the solutions (11) into eq. (6) we obtain that

$$
L \alpha \gamma_{2}=M\left(\alpha \gamma_{2}+2(1-q) \rho\right)
$$

For these values of the parameters $\alpha, \beta$ and $\delta$ and the constants $K, L$ and $M$ eq. (9) on the boundary $t=0$ is also satisfied. The results can now be summarized as follows. Let

$$
\begin{aligned}
& \alpha_{0}=\rho^{2}, \quad \beta_{1}=X_{-}\left(\alpha_{0}\right), \quad \beta_{2}=x_{-}\left(\alpha_{0}\right), \\
& d_{1}=\frac{\alpha_{0} \gamma_{1}+2 q \rho}{\alpha_{0} \gamma_{1}}, \quad d_{2}=\frac{\alpha_{0} \gamma_{2}+2(1-q) \rho}{\alpha_{0} \gamma_{2}}, \quad e_{0}=1,
\end{aligned}
$$

and set for all $s \geqslant 0$ and $t \geqslant 1$,

$$
X_{s, t}^{0}=d_{1} \alpha_{0}^{s} \beta_{1}^{t}, \quad x_{s, t}^{0}=d_{2} \alpha_{0}^{s} \beta_{2}^{t},
$$

and for all $s \geqslant 0$ and $t=0$,

$$
X_{s, 0}^{0}=x_{s, 0}^{0}=e_{0} \alpha_{0}^{s} .
$$

Then we empirically find as $s \rightarrow \infty$ and $t \geqslant 0$ that

$$
Q_{s, t} \sim M X_{s, t}^{0}, \quad q_{s, t} \sim M x_{s, t}^{0},
$$


for some constant $M$. The asymptotic solutions $X_{s, t}^{0}$ and $x_{s, t}^{0}$ satisfy all equilibrium equations, except the equations on the boundary $s=0$. Therefore, these asymptotic solutions do not describe the behaviour of the equilibrium probabilities near the boundary $s=0$. In the next section we show how these initial asymptotic solutions can be improved by adding terms to compensate for the error on the boundary $s=0$.

\section{Compensation on the boundary $s=0$}

To find a proper compensation of the initial asymptotic solution $X_{s, t}^{0}$ on the boundary $s=0$, we form the linear combination $X_{s, t}^{0}+d_{1} c_{1} \alpha^{s} \beta^{t}$. We try to choose $c_{1}, \alpha$ and $\beta$ such that this linear combination satisfies eqs. (3) and (1). Inserting this linear combination into eq. (3) and dividing both sides by $d_{1}$, yields for all $t>1$ that

$$
\left(\beta_{1}^{t}+c_{1} \beta^{t}\right)\left(2 \rho+\gamma_{2}\right)=\left(\beta_{1}^{t+1}+c_{1} \beta^{t+1}\right) \gamma_{2}+\left(\alpha_{0} \beta_{1}^{t-1}+c_{1} \alpha \beta^{t-1}\right) \gamma_{1}
$$

Since this must hold for all $t>1$, we set $\beta=\beta_{1}$. We require that $\alpha^{s} \beta_{1}^{t}$ satisfies eq. (1), so by linearity, $X_{s, t}^{0}+d_{1} c_{1} \alpha^{s} \beta_{1}^{t}$ satisfies (1). By lemma 1(i), the term $\alpha^{s} \beta_{1}^{t}$ satisfies eq. (1) for $\alpha=Y_{+}\left(\beta_{1}\right)\left(=\alpha_{0}\right)$ and $\alpha=Y_{-}\left(\beta_{1}\right)$. Hence, we set $\alpha=\alpha_{1}=$ $Y_{-}\left(\beta_{1}\right)$. Dividing the above equation by the common factor $\beta_{1}^{t-1}$ yields a single equation for $c_{1}$. Hence, we can choose $c_{1}$ such that the equations on the boundary $s=0$ are satisfied. Accordingly we compensate for the error of $x_{s, t}^{0}$ on the boundary $s=0$. In general the result can be stated as

LEMMA 2

(i) Let for all $s \geqslant 0$ and $t \geqslant 1$,

$$
Z_{s, t}=k_{1} Y_{+}^{s}(\beta) \beta^{t}+k_{2} Y_{-}^{s}(\beta) \beta^{t} .
$$

Then $Z_{s, t}$ satisfies eqs. (1) and (3) if $k_{1}$ and $k_{2}$ satisfy

$$
k_{2}=-\frac{Y_{-}(\beta)-\beta}{Y_{+}(\beta)-\beta} k_{1} \text {. }
$$

(ii) Let for all $s \geqslant 0$ and $t \geqslant 1$,

$$
z_{s, t}=k_{1} y_{+}^{s}(\beta) \beta^{t}+k_{2} y_{-}^{s}(\beta) \beta^{t} .
$$

Then $z_{s, t}$ satisfies eqs. (5) and (7) if $k_{1}$ and $k_{2}$ satisfy

$$
k_{2}=-\frac{y_{-}(\beta)-\beta}{y_{+}(\beta)-\beta} k_{1} .
$$

\section{Proof}

We prove part (i). The proof of (ii) is similar. By lemma 1(i) the terms $Y_{+}^{s}(\beta) \beta^{\imath}$ and $Y_{-}^{s}(\beta) \beta^{t}$ satisfy eq. (1). Since eq. (1) is linear, any linear combination also 
satisfies (1). Inserting $Z_{s, t}$ into (3) and dividing by the common term $\beta^{t-1}$ leads to

$$
\left(k_{1}+k_{2}\right) \beta\left(2 \rho+\gamma_{2}\right)=\left(k_{1}+k_{2}\right) \beta^{2} \gamma_{2}+k_{1} Y_{+}(\beta) \gamma_{1}+k_{2} Y_{-}(\beta) \gamma_{1},
$$

which can be rewritten as

$$
k_{2}=-\frac{\beta\left(2 \rho+\gamma_{2}\right)-\beta^{2} \gamma_{2}-Y_{+}(\beta) \gamma_{1}}{\beta\left(2 \rho+\gamma_{2}\right)-\beta^{2} \gamma_{2}-Y_{-}(\beta) \gamma_{1}} k_{1}
$$

Since $Y_{-}(\beta)$ and $Y_{+}(\beta)$ are the roots of (13),

$$
Y_{-}(\beta) \gamma_{1}+Y_{+}(\beta) \gamma_{1}=\beta 2(\rho+1)-\beta^{2} \gamma_{2} \text {. }
$$

Substituting that equality into (16) yields relation (15).

Set for all $s \geqslant 0$ and $t \geqslant 1$,

$$
X_{s, t}^{1}=X_{s, t}^{0}+d_{1} c_{1} \alpha_{1}^{s} \beta_{1}^{t}, \quad x_{s, t}^{1}=x_{s, t}^{0}+d_{2} c_{2} \alpha_{2}^{s} \beta_{2}^{t},
$$

and for all $s \geqslant 0$ and $t=0$,

$$
X_{s, 0}^{1}=x_{s, 0}^{1}=x_{s, 0}^{0} \text {. }
$$

The term $d_{2} c_{2} \alpha_{2}^{s} \beta_{2}^{t}$ is added to $x_{s, t}^{0}$ to compensate for the error of $x_{s, t}^{0}$ on the boundary $s=0$. Here $\alpha_{2}=y_{-}\left(\beta_{2}\right)$. The proper coefficients follow from lemma 2 . Numerical experience suggests that $X_{s, t}^{1}$ and $x_{s, t}^{1}$ also describe the behaviour of the equilibrium probabilities near the boundary $s=0$. Therefore we empirically find as $s+t \rightarrow \infty$ that,

$$
Q_{s, t} \sim M X_{s, t}^{\mathrm{1}}, \quad q_{s, t} \sim M x_{s, t}^{1},
$$

for some constant $M$. In section 10 we rigorously prove that asymptotic result. We added extra terms to the initial solutions $X_{s, t}^{0}$ and $x_{s, t}^{0}$ to compensate for the error on the boundary $s=0$. On the other hand, we introduced new errors on the boundaries $t=0$ and $t=1$, since the extra compensation terms violate eqs. (2), (6) and (9). In the next section we show how to compensate for these errors.

\section{Compensation on the boundaries $t=0$ and $t=1$}

To compensate for the error introduced by $d_{1} c_{1} \alpha_{1}^{s} \beta_{1}^{t}$ in $X_{s, t}^{1}$ on the boundaries $t=0$ and $t=1$, we add the compensation term $d_{3} \alpha_{1}^{s} \beta^{t}$ to $X_{s, t}^{1}$, the term $e_{1} \alpha_{1}^{s}$ to $X_{s, 0}^{1}$ and $d_{4} \alpha_{1}^{s} \delta^{t}$ to $x_{s, t}^{1}$. The compensation terms all have the same $\alpha$-factor as the error term $d_{1} c_{1} \alpha_{1}^{s} \beta_{1}^{t}$. This follows from the requirement that eqs. (2), (6) and (9) on the boundaries $t=0$ and $t=1$ must be satisfied for all $s>0$. Compare the compensation on the boundaries $s=0$, where the compensation term has the same $\beta$-factor as the error term. It explains why we cannot simultaneously compensate for the errors introduced by $d_{1} c_{1} \alpha_{1}^{s} \beta_{1}^{t}$ in $X_{s, t}^{1}$ and $d_{2} c_{2} \alpha_{2}^{s} \beta_{2}^{t}$ in $x_{s, t}^{1}$, 
since these error terms have a different $\alpha$-factor. We require that the compensation term $d_{3} \alpha_{1}^{s} \beta^{t}$ satisfies eq. (1) and $d_{4} \alpha_{1}^{s} \delta^{t}$ satisfies eq. (5), so by linearity, $X_{s, t}^{1}+d_{3} \alpha_{1}^{s} \beta^{t}$ satisfies (1) and $x_{s, t}^{1}+d_{4} \alpha_{1}^{s} \delta^{t}$ satisfies (5). By lemma $1(\mathrm{i})$, the term $d_{3} \alpha_{1}^{s} \beta^{t}$ satisfies eq. (1) for $\beta=X_{+}\left(\alpha_{1}\right)\left(=\beta_{1}\right)$ and $\beta=X_{-}\left(\alpha_{1}\right)$. Hence, we set $\beta=\beta_{3}=X_{-}\left(\alpha_{1}\right)$. Accordingly, we set $\delta=\beta_{4}=x_{-}\left(\alpha_{1}\right)$. Here we take the smaller root, since the compensation term $\alpha_{1}^{s} \delta^{t}$ must be as small as possible. Inserting the error term together with the compensation terms into eqs. (2), (6) and (9) and then dividing these equations by the common factor $\alpha_{1}^{s-1}$, yields three equations for the coefficients $d_{3}, e_{1}$ and $d_{4}$. Hence, we can choose these coefficients such that the equations on the boundaries $t=0$ and $t=1$ are satisfied. Accordingly we can compensate for the error introduced by $d_{2} c_{2} \alpha_{2}^{s} \beta_{2}^{t}$ in $x_{s, t}^{1}$. In general, the result can be stated as

LEMMA 3

(i) Let for all $s \geqslant 0$ and $t \geqslant 1$,

$$
\begin{aligned}
& Z_{s, t}=k_{1} \alpha^{s} X_{+}^{t}(\alpha)+k_{2} \alpha^{s} X_{-}^{t}(\alpha), \\
& z_{s, t}=k_{3} \alpha^{s} x_{-}^{t}(\alpha),
\end{aligned}
$$

and for all $s \geqslant 0$,

$$
Z_{s, 0}=z_{s, 0}=k_{4} \alpha^{s} \text {. }
$$

Then $Z_{s, t}$ and $z_{s, t}$ satisfy eqs. (1), (2), (5), (6) and (9) if $k_{1}, k_{2}, k_{3}$ and $k_{4}$ satisfy

$$
\begin{gathered}
k_{2}=-\frac{\frac{\alpha \gamma_{1}+2 q \rho}{X_{-}(\alpha)}+\frac{\alpha \gamma_{2}+2(1-q) \rho}{x_{+}(\alpha)}-2(\rho+1)}{\frac{\alpha \gamma_{1}+2 q \rho}{X_{+}(\alpha)}+\frac{\alpha \gamma_{2}+2(1-q) \rho}{x_{+}(\alpha)}-2(\rho+1)} k_{1}, \\
k_{3}=-\frac{\gamma_{1}\left(\alpha \gamma_{2}+2(1-q) \rho\right)\left(\frac{1}{X_{-}(\alpha)}-\frac{1}{X_{+}(\alpha)}\right)}{\gamma_{2}\left(\frac{\alpha \gamma_{1}+2 q \rho}{X_{+}(\alpha)}+\frac{\alpha \gamma_{2}+2(1-q) \rho}{x_{+}(\alpha)}-2(\rho+1)\right)} k_{1}, \\
k_{4}=\left(k_{1}+k_{2}\right) \frac{\alpha \gamma_{1}}{\alpha \gamma_{1}+2 q \rho}=k_{3} \frac{\alpha \gamma_{2}}{\alpha \gamma_{2}+2(1-q) \rho} .
\end{gathered}
$$

(ii) Let for all $s \geqslant 0$ and $t \geqslant 1$,

$$
\begin{aligned}
& z_{s, t}=k_{1} \alpha^{s} x_{+}^{t}(\alpha)+k_{2} \alpha^{s} x_{-}^{t}(\alpha), \\
& Z_{s, t}=k_{3} \alpha^{s} X_{-}^{t}(\alpha),
\end{aligned}
$$

and for all $s \geqslant 0$,

$$
z_{s, 0}=Z_{s, 0}=k_{4} \alpha^{s}
$$


Then $z_{s, t}$ and $Z_{s, t}$ satisfy eqs. (1), (2), (5), (6) and (9) if $k_{1}, k_{2}, k_{3}$ and $k_{4}$ satisfy

$$
\begin{gathered}
k_{2}=-\frac{\frac{\alpha \gamma_{1}+2 q \rho}{X_{+}(\alpha)}+\frac{\alpha \gamma_{2}+2(1-q) \rho}{x_{-}(\alpha)}-2(\rho+1)}{\frac{\alpha \gamma_{1}+2 q \rho}{X_{+}(\alpha)}+\frac{\alpha \gamma_{2}+2(1-q) \rho}{x_{+}(\alpha)}-2(\rho+1)} k_{1}, \\
k_{3}=-\frac{\gamma_{2}\left(\alpha \gamma_{1}+2 q \rho\right)\left(\frac{1}{x_{-}(\alpha)}-\frac{1}{x_{+}(\alpha)}\right)}{\gamma_{1}\left(\frac{\alpha \gamma_{1}+2 q \rho}{X_{+}(\alpha)}+\frac{\alpha \gamma_{2}+2(1-q) \rho}{x_{+}(\alpha)}-2(\rho+1)\right)} k_{1}, \\
k_{4}=\left(k_{1}+k_{2}\right) \frac{\alpha \gamma_{2}}{\alpha \gamma_{2}+2(1-q) \rho}=k_{3} \frac{\alpha \gamma_{1}}{\alpha \gamma_{2}+2 q \rho} .
\end{gathered}
$$

\section{Proof}

We prove part (i). The proof of (ii) is similar. By lemma 1(i) both $\alpha^{s} X_{+}^{t}(\alpha)$ and $\alpha^{s} X_{-}^{t}(\alpha)$ satisfy eq. (1). Since eq. (1) is linear, $Z_{s, t}$ also satisfies (1). Accordingly, $z_{s, t}$ satisfies eq. (5). Inserting $Z_{s, t}$ into eq. (2) and dividing both sides by the common factor $\alpha^{s-1}$ yields that

$$
\begin{aligned}
{\left[k_{1} \alpha X_{+}(\alpha)+k_{2} \alpha X_{-}(\alpha)\right] 2(\rho+1)=} & {\left[k_{1} X_{+}^{2}(\alpha)+k_{2} X_{-}^{2}(\alpha)\right]\left(2 \rho+\alpha \gamma_{2}\right) } \\
& +k_{4}\left(\alpha^{2} \gamma_{1}+\alpha 2 q \rho\right) .
\end{aligned}
$$

By inserting the quadratic equation (13) this reduces to

$$
\left(k_{1}+k_{2}\right) \alpha \gamma_{1}=k_{4}\left(\alpha \gamma_{1}+2 q \rho\right) \text {. }
$$

Accordingly, inserting $z_{s, t}$ into eq. (6) leads to

$$
k_{3} \alpha \gamma_{2}=k_{4}\left(\alpha \gamma_{2}+2(1-q) \rho\right)
$$

The equalities (20) and (21) prove (19), and combining these equalities gives

$$
k_{3} \gamma_{2}\left(\alpha \gamma_{1}+2 q \rho\right)=\left(k_{1}+k_{2}\right) \gamma_{1}\left(\alpha \gamma_{2}+2(1-q) \rho\right) \text {. }
$$

Inserting $Z_{s, t}$ and $z_{s, t}$ into eq. (9) and dividing both sides by $\alpha^{s-1}$ yields

$$
k_{4} \alpha 2(\rho+1)=\left[k_{1} X_{+}(\alpha)+k_{2} X_{-}(\alpha)\right]\left(2 \rho+\alpha \gamma_{2}\right)+k_{3} x_{-}(\alpha)\left(2 \rho+\alpha \gamma_{1}\right) \text {. }
$$

Since $X_{+}(\alpha)$ and $X_{-}(\alpha)$ are the roots of the quadratic equation (13),

$$
X_{+}(\alpha) X_{-}(\alpha)\left(2 \rho+\alpha \gamma_{2}\right)=\alpha^{2} \gamma_{1}
$$

so we have that

$$
\begin{aligned}
& X_{+}(\alpha)=\frac{\alpha^{2} \gamma_{1}}{X_{-}(\alpha)\left(2 \rho+\alpha \gamma_{2}\right)} \\
& X_{-}(\alpha)=\frac{\alpha^{2} \gamma_{1}}{X_{+}(\alpha)\left(2 \rho+\alpha \gamma_{2}\right)}
\end{aligned}
$$


Accordingly,

$$
x_{-}(\alpha)=\frac{\alpha^{2} \gamma_{2}}{x_{+}(\alpha)\left(2 \rho+\alpha \gamma_{1}\right)} .
$$

Inserting these equalities into (23) and eliminating $k_{3}$ and $k_{4}$ by inserting (20) and (22) leads to the desired relation (17). Relation (18) follows by inserting (17) into (22).

Set for all $s \geqslant 0$ and $t \geqslant 1$,

$$
\begin{aligned}
& X_{s, t}^{2}=X_{s, t}^{1}+d_{3} \alpha_{1}^{s} \beta_{3}^{t}+d_{5} \alpha_{2}^{s} \beta_{5}^{t}, \\
& x_{s, t}^{2}=x_{s, t}^{1}+d_{4} \alpha_{1}^{s} \beta_{4}^{t}+d_{6} \alpha_{2}^{s} \beta_{6}^{t},
\end{aligned}
$$

and for all $s \geqslant 0$ and $t=0$,

$$
X_{s, 0}^{2}=X_{s, 0}^{1}+e_{1} \alpha_{1}^{s}+e_{2} \alpha_{2}^{s} .
$$

The terms $d_{5} \alpha_{2}^{s} \beta_{5}^{t}, d_{6} \alpha_{2}^{s} \beta_{6}^{t}$ and $e_{2} \alpha_{2}^{s}$ are added to compensate for the error introduced by $d_{2} c_{2} \alpha_{2}^{s} \beta_{2}^{t}$ in $x_{s, t}^{1}$. Here $\beta_{5}=X_{-}\left(\alpha_{2}\right)$ and $\beta_{6}=x_{-}\left(\alpha_{2}\right)$. The proper coefficients follow from lemma 3 . We compensated for the errors on the boundaries $t=0$ and $t=1$, but we introduced new errors on the boundary $s=0$, since the extra compensation terms $d_{3} \alpha_{1}^{s} \beta_{3}^{t}$ and $d_{5} \alpha_{2}^{s} \beta_{5}^{t}$ in the upper triangle violate eq. (3) and the extra compensation terms in the lower triangle violate eq. (7). But it is clear how to continue this compensation procedure: it consists of adding on terms so as to compensate alternatingly for the error on the boundary $s=0$ according to lemma 2, and for the error on the boundaries $t=0$ and $t=1$ according to lemma 3 . Then a sequence of asymptotic solutions $X_{s, t}^{k}$ and $x_{s, t}^{k}$, $k=0,1,2, \ldots$, is generated, where $X_{s, t}^{k}$ and $x_{s, t}^{k}$ are the solutions after $k$ compensation steps. Because of the compensation on the boundaries $t=0$ and $t=1$, the solutions $X_{s, t}^{k}$ and $x_{s, t}^{k}$ grow as a binary tree of compensation terms as $k \rightarrow \infty$. The problem is to prove that the sequence of $X_{s, t}^{k}$ and $x_{s, t}^{k}$ converges. Before treating the convergence, we formally define the final solutions $X_{s, t}=$ $\lim _{k \rightarrow \infty} X_{s, t}^{k}$ and $x_{s, t}=\lim _{\mathrm{k} \rightarrow \infty} x_{s, t}^{k}$.

\section{Formal definition of the compensation procedure}

The final solutions $X_{s, t}$ and $x_{s, t}$ consist of an infinite linear combination of solutions of the form $\alpha^{s} \beta^{t}$. We first define the parameters $\alpha_{i}$ and $\beta_{i}$, and then the coefficients for the infinite linear combinations. The parameters $\alpha_{i}$ and $\beta_{i}$ can be represented in a tree as depicted in fig. 2 . That tree will be called the parameter tree. In fig. 2 the $\alpha$ 's and $\beta$ 's are numbered from the root and from left to right. Actually, the numbering of the $\alpha$ 's and $\beta$ 's is irrelevant: we only use that the $\alpha$-root has index 0 and that the other $\alpha$ 's have the same index as their $\beta$-parent. 


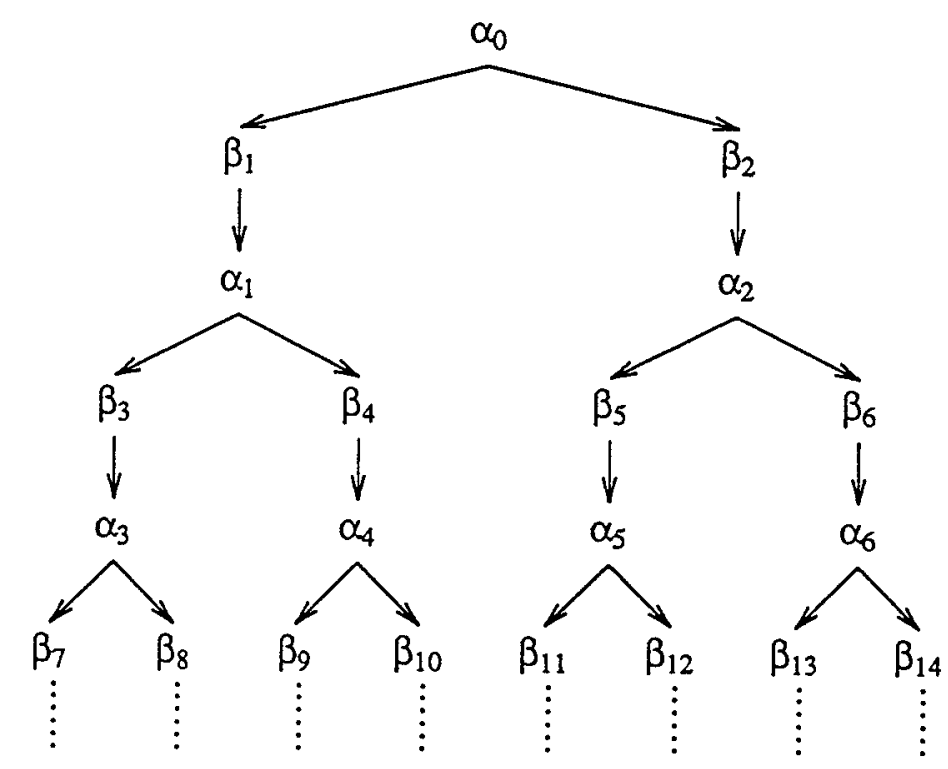

Fig. 2. The parameter tree of numbers $\alpha_{i}$ and $\beta_{i}$.

For specifying the recursion relations to generate the tree, we need the following notation.

$\beta_{l(i)}=$ the left descendant of $\alpha_{i}$,

$\beta_{r(i)}=$ the right descendant of $\alpha_{i}$,

$\alpha_{p(i)}=$ the $\alpha$-parent of $\beta_{i}$.

Further, define $L$ as the set of indices $i$ of $\beta_{i}$ 's which are a left descendant and $R$ as the set of indices $i$ of $\beta_{i}$ 's which are a right descendant, that is,

$$
\begin{aligned}
& L=\{l(i), i=0,1,2, \ldots\}, \\
& R=\{r(i), i=0,1,2, \ldots\} .
\end{aligned}
$$

The numbers $\alpha_{i}$ and $\beta_{i}$ are defined as roots of the quadratic equations in the upper and lower triangle. For the roots of these quadratic equations, it is easy to prove that for all $0<\alpha \leqslant \rho^{2}$ (see also lemma 4 in section 8),

$$
\begin{aligned}
& X_{+}(\alpha)>\alpha>X_{-}(\alpha)>0, \\
& x_{+}(\alpha)>\alpha>x_{-}(\alpha)>0,
\end{aligned}
$$

and for all $0<\beta \leqslant \rho$,

$$
\begin{aligned}
& Y_{+}(\beta)>\beta>Y_{-}(\beta)>0, \\
& y_{+}(\beta)>\beta>y_{-}(\beta)>0 .
\end{aligned}
$$

Initially set $\alpha_{0}=\rho^{2}$. Then for all $i \geqslant 0$ the left descendant $\beta_{l(i)}$ of $\alpha_{i}$ is defined as the smaller root of eq. (13) for fixed $\alpha=\alpha_{i}$ and the descendant $\alpha_{l(i)}$ of $\beta_{l(i)}$ as the smaller root of (13) for fixed $\beta=\beta_{l(i)}$. The right descendant $\beta_{r(i)}$ of $\alpha_{i}$ is defined 
as the smaller root of eq. (14) for fixed $\alpha=\alpha_{i}$ and the descendant $\alpha_{r(i)}$ of $\beta_{r(i)}$ as the smaller root of (14) for fixed $\beta=\beta_{r(i)}$. From the inequalities (24) it follows by induction that for all $i \geqslant 0$,

$$
\begin{array}{ll}
\beta_{l(i)}=X_{-}\left(\alpha_{i}\right), & \alpha_{l(i)}=Y_{-}\left(\beta_{l(i)}\right), \\
\beta_{r(i)}=x_{-}\left(\alpha_{i}\right), & \alpha_{r(i)}=y_{-}\left(\beta_{r(i)}\right),
\end{array}
$$

and that

$$
\alpha_{i}>\beta_{l(i)}>\alpha_{l(i)}>0, \quad \alpha_{i}>\beta_{r(i)}>\alpha_{r(i)}>0 .
$$

So $\alpha_{i}$ and $\beta_{i}$ form a decreasing positive tree. For all $i \in L$ the pairs $\alpha_{p(i)}$ and $\beta_{i}$, as well as $\beta_{i}$ and $\alpha_{i}$, satisfy the quadratic equation (13). Hence, by lemma 1(i), for all $i \in L$ the products $\alpha_{p(i)}^{s} \beta_{i}^{t}$ and $\alpha_{i}^{s} \beta_{i}^{t}$ satisfy eq. (1). Accordingly, for all $i \in R$ the products $\alpha_{p(i)}^{s} \beta_{i}^{t}$ and $\alpha_{i}^{s} \beta_{i}^{t}$ satisfy eq. (5). Since eqs. (1) and (5) are linear, any linear combination of products $\alpha_{p(i)}^{s} \beta_{i}^{t}$ and $\alpha_{i}^{s} \beta_{i}^{t}$ with $i \in L$ satisfies eq. (1) and any linear combination of products $\alpha_{p(i)}^{s} \beta_{i}^{t}$ and $\alpha_{i}^{s} \beta_{i}^{t}$ with $i \in R$ satisfies eq. (5). Now form the infinite linear combinations $X_{s, t}$ and $x_{s, t}$, for all $s \geqslant 0$ and $t \geqslant 1$ defined as

$$
\begin{aligned}
& X_{s, t}=\sum_{i \in L} d_{i}\left(\alpha_{p(i)}^{s}+c_{i} \alpha_{i}^{s}\right) \beta_{i}^{t}, \\
& x_{s, t}=\sum_{i \in R} d_{i}\left(\alpha_{p(i)}^{s}+c_{i} \alpha_{i}^{s}\right) \beta_{i}^{t} .
\end{aligned}
$$

The sums (25) and (26) can be represented in a binary tree, derived from the original parameter tree. The binary tree of terms $d_{i}\left(\alpha_{p(i)}^{s}+c_{i} \alpha_{i}^{s}\right) \beta_{i}^{t}, i \geqslant 1$, will be called the compensation tree. In fig. 3 we depict the compensation tree. The left descendants $d_{i}\left(\alpha_{p(i)}^{s}+c_{i} \alpha_{i}^{s}\right) \beta_{i}^{t}$ in the compensation tree are those for which $i \in L$. Hence, (25) is the sum of all left descendants in the compensation tree and (26) is the sum of all right descendants. In both sums (25) and (26), we formed pairs of products with a common $\beta$-factor, which is required for the error correction on the boundary $s=0$. The coefficients $c_{i}$ are generated such that the terms $\left(\alpha_{p(i)}^{s}+c_{i} \alpha_{i}^{s}\right) \beta_{i}^{t}$ satisfy eq. (3) for all $i \in L$ and eq. (7) for all $i \in R$. Since

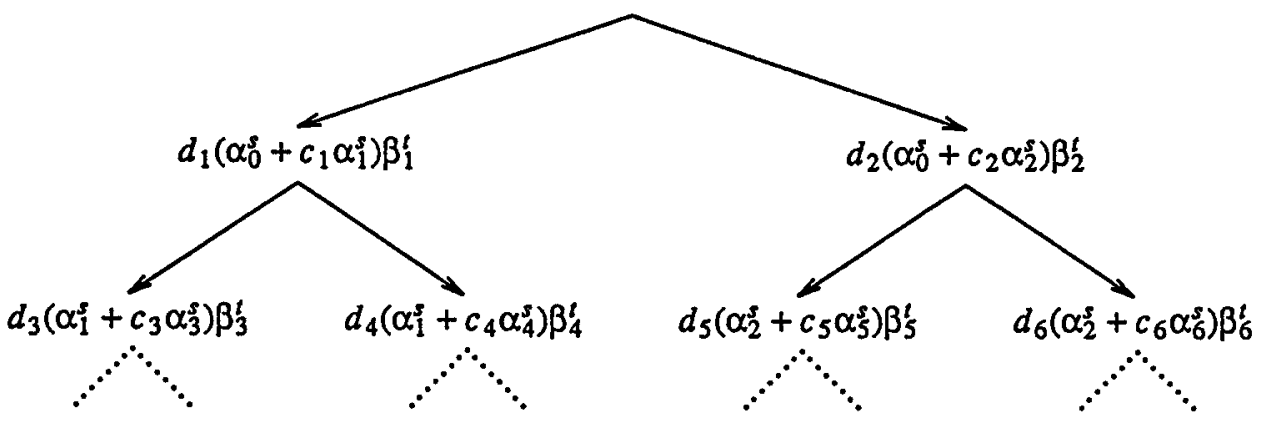

Fig. 3. The compensation tree of terms $d_{i}\left(\alpha_{p(i)}^{s}+c_{i} \alpha_{i}^{s}\right) \beta_{i}^{t}$. 
$\alpha_{p(i)}=Y_{+}\left(\beta_{i}\right)$ and $\alpha_{i}=Y_{-}\left(\beta_{i}\right)$ for all $i \in L$, we obtain by lemma 2(i) for all $i \in L$ that,

$$
c_{i}=-\frac{Y_{-}\left(\beta_{i}\right)-\beta_{i}}{Y_{+}\left(\beta_{i}\right)-\beta_{i}}
$$

and accordingly, by lemma 2(ii) for all $i \in R$ that,

$$
c_{i}=-\frac{y_{-}\left(\beta_{i}\right)-\beta_{i}}{y_{+}\left(\beta_{i}\right)-\beta_{i}} .
$$

Since eqs. (3) and (7) are linear, the linear combination (25) satisfies eq. (3) and the linear combination (26) eq. (7). The error correction on the boundaries $t=0$ and $t=1$ requires pairs of products with a common $\alpha$-factor. Therefore we rewrite the sums (25) and (26) as

$$
\begin{aligned}
& X_{s, t}=d_{1} \beta_{1}^{t} \alpha_{0}^{s}+\sum_{i \in L}\left(d_{i} c_{i} \beta_{i}^{t}+d_{l(i)} \beta_{l(i)}^{t}\right) \alpha_{i}^{s}+\sum_{i \in R} d_{l(i)} \beta_{l(i)}^{t} \alpha_{i}^{s}, \\
& x_{s, t}=d_{2} \beta_{2}^{t} \alpha_{0}^{s}+\sum_{i \in L} d_{r(i)} \beta_{r(i)}^{t} \alpha_{i}^{s}+\sum_{i \in R}\left(d_{i} c_{i} \beta_{i}^{t}+d_{r(i)} \beta_{r(i)}^{t}\right) \alpha_{i}^{s}
\end{aligned}
$$

and define for all $s \geqslant 0$ and $t=0$,

$$
X_{s, 0}=x_{s, 0}=\sum_{i=0}^{\infty} e_{i} \alpha_{i}^{s}=e_{0} \alpha_{0}^{s}+\sum_{i \in L} e_{i} \alpha_{i}^{s}+\sum_{i \in R} e_{i} \alpha_{i}^{s} .
$$

The coefficients $d_{i}$ and $e_{i}$ are generated such that $X_{s, t}$ and $x_{s, t}$ satisfy the equations on the boundaries $t=0$ and $t=1$. Initially set (cf. section 3 )

$$
\begin{aligned}
& d_{1}=\frac{\alpha_{0} \gamma_{1}+2 q \rho}{\alpha_{0} \gamma_{1}} \\
& d_{2}=\frac{\alpha_{2} \gamma_{2}+2(1-q) \rho}{\alpha_{0} \gamma_{2}} \\
& e_{0}=1 .
\end{aligned}
$$

Then the coefficients $d_{l(i)}, d_{r(i)}$ and $e_{i}$ are generated such that for all $i \in L$ the terms $\left(d_{i} c_{i} \beta_{i}^{t}+d_{l(i)} \beta_{l(i)}^{t}\right) \alpha_{i}^{s}$ and $d_{r(i)} \beta_{r(i)}^{t} \alpha_{i}^{s}$ and $e_{i} \alpha_{i}^{s}$ satisfy eqs. (2), (6) and (9) and for all $i \in R$ the terms $d_{t(i)} \beta_{l(i)}^{t} \alpha_{i}^{s}$ and $\left(d_{i} c_{i} \beta_{i}^{t}+d_{r(i)} \beta_{r(i)}^{t}\right) \alpha_{i}^{s}$ and $e_{i} \alpha_{i}^{s}$ satisfy eqs. (2), (6) and (9). Since $\beta_{l(i)}=X_{-}\left(\alpha_{i}\right), \beta_{r(i)}=x_{-}\left(\alpha_{i}\right)$ and $\beta_{i}=X_{+}\left(\alpha_{i}\right)$ for all $i \in L$, we obtain by lemma 3(i) for all $i \in L$ that,

$$
d_{l(i)}=-\frac{\frac{\alpha_{i} \gamma_{1}+2 q \rho}{X_{-}\left(\alpha_{i}\right)}+\frac{\alpha_{i} \gamma_{2}+2(1-q) \rho}{x_{+}\left(\alpha_{i}\right)}-2(\rho+1)}{\frac{\alpha_{i} \gamma_{1}+2 q \rho}{X_{+}\left(\alpha_{i}\right)}+\frac{\alpha_{i} \gamma_{2}+2(1-q) \rho}{x_{+}\left(\alpha_{i}\right)}-2(\rho+1)} d_{i} c_{i}
$$




$$
\begin{aligned}
& d_{r(i)}=-\frac{\gamma_{1}\left(\alpha_{i} \gamma_{2}+2(1-q) \rho\right)\left(\frac{1}{X_{-}\left(\alpha_{i}\right)}-\frac{1}{X_{+}\left(\alpha_{i}\right)}\right)}{\gamma_{2}\left(\frac{\alpha_{i} \gamma_{1}+2 q \rho}{X_{+}\left(\alpha_{i}\right)}+\frac{\alpha_{i} \gamma_{2}+2(1-q) \rho}{x_{+}\left(\alpha_{i}\right)}-2(\rho+1)\right)} d_{i} c_{i}, \\
& e_{i}=\left(d_{i} c_{i}+d_{l(i)}\right) \frac{\alpha_{i} \gamma_{1}}{\alpha_{i} \gamma_{1}+2 q \rho}=d_{r(i)} \frac{\alpha_{i} \gamma_{2}}{\alpha_{i} \gamma_{2}+2(1-q) \rho},
\end{aligned}
$$

and accordingly, by lemma 3 (ii) for all $i \in R$ that,

$$
\begin{aligned}
& d_{l(i)}=-\frac{\gamma_{2}\left(\alpha_{i} \gamma_{1}+2 q \rho\right)\left(\frac{1}{x_{-}\left(\alpha_{i}\right)}-\frac{1}{x_{+}\left(\alpha_{i}\right)}\right)}{\gamma_{1}\left(\frac{\alpha_{i} \gamma_{1}+2 q \rho}{X_{+}\left(\alpha_{i}\right)}+\frac{\alpha_{i} \gamma_{2}+2(1-q) \rho}{x_{+}\left(\alpha_{i}\right)}-2(\rho+1)\right)} d_{i} c_{i}, \\
& d_{r(i)}=-\frac{\frac{\alpha_{i} \gamma_{1}+2 q \rho}{X_{+}\left(\alpha_{i}\right)}+\frac{\alpha_{i} \gamma_{2}+2(1-q) \rho}{x_{-}\left(\alpha_{i}\right)}-2(\rho+1)}{\frac{\alpha_{i} \gamma_{1}+2 q \rho}{X_{+}\left(\alpha_{i}\right)}+\frac{\alpha_{i} \gamma_{2}+2(1-q) \rho}{x_{+}\left(\alpha_{i}\right)}-2(\rho+1)} d_{i} c_{i}, \\
& e_{i}=\left(d_{i} c_{i}+d_{r(i)}\right) \frac{\alpha_{i} \gamma_{2}}{\alpha_{i} \gamma_{2}+2(1-q) \rho}=d_{l(i)} \frac{\alpha_{i} \gamma_{1}}{\alpha_{i} \gamma_{1}+2 q \rho} .
\end{aligned}
$$

Since eqs. (2), (6) and (9) are linear, the linear combinations (27), (28) and (29) satisfy eqs. (2), (6) and (9). This completes the formal definition of the numbers $X_{s, t}$ and $x_{s, t}$ for all $s \geqslant 0$ and $t \geqslant 0$.

It is obvious that the final solutions $X_{s, t}$ and $x_{s, t}$ formally satisfy all equations, except eqs. (4), (8) and (10). To see that these equations are also satisfied, insert the series (25) into eq. (4). This leads to

$$
\sum_{i \in L}\left(\left(1+c_{i}\right) \beta_{i}\left(2 \rho+\gamma_{2}\right)-\left(1+c_{i}\right) \beta_{i}^{2} \gamma_{2}\right)=X_{1,0} \gamma_{1}+X_{0,0} 2 q \rho
$$

We determined $c_{i}$ such that for all $i \in L$ the terms $\left(\alpha_{p(i)}^{s}+c_{i} \alpha_{i}^{s}\right) \beta_{i}^{t}$ satisfy eq. (3), that is, for all $t>1$,

$$
\left(1+c_{i}\right) \beta_{i}^{t}\left(2 \rho+\gamma_{2}\right)-\left(1+c_{i}\right) \beta_{i}^{t+1} \gamma_{2}=\left(\alpha_{p(i)}+c_{i} \alpha_{i}\right) \beta_{i}^{t-1} \gamma_{1} .
$$

Dividing both sides by $\beta_{i}^{t-1}$ and then inserting into (30) we obtain that

$$
\sum_{i \in L}\left(\alpha_{p(i)}+c_{i} \alpha_{i}\right) \gamma_{1}=X_{1,0} \gamma_{1}+X_{0,0} 2 q \rho \text {. }
$$

Then note that by definition for all $s \geqslant 0$,

$$
\begin{aligned}
X_{s, 0} & =\alpha_{0}^{s}+\sum_{i \in L}\left(d_{i} c_{i}+d_{l(i)}\right) \frac{\alpha_{i}^{s+1} \gamma_{1}}{\alpha_{i} \gamma_{1}+2 q \rho}+\sum_{i \in R} d_{l(i)} \frac{\alpha_{i}^{s+1} \gamma_{1}}{\alpha_{i} \gamma_{1}+2 q \rho} \\
& =\sum_{i \in L} d_{i}\left(\frac{\alpha_{p(i}^{s+1} \gamma_{1}}{\alpha_{p(i)} \gamma_{1}+2 q \rho}+c_{i} \frac{\alpha_{i}^{s+1} \gamma_{1}}{\alpha_{i} \gamma_{1}+2 q \rho}\right) .
\end{aligned}
$$


By inserting these series for $X_{1,0}$ and $X_{0,0}$ eq. (31) reduces to an identity. It can be similarly seen that eq. (8) is satisfied. The remaining equation in $(0,0)$ is also satisfied, since summing over all other equations and then changing summations yields the desired equation.

\section{Remark 1 (identical servers)}

The analysis essentially simplifies if $\gamma_{1}=\gamma_{2}$. Then the quadratic equations in the upper and lower triangle are identical. Hence, in the parameter tree all $\alpha$ 's at the same depth and all $\beta$ 's at the same depth are equal, so the binary tree generation of the $\alpha$ 's and $\beta$ 's simplifies to the generation of a sequence of $\alpha$ 's and $\beta$ 's, which has the structure<smiles>[Te][Te][Te][Te]</smiles>

As a consequence, the series for $X_{s, t}$ and $x_{s, t}$ have a linear structure. The problem with identical servers is worked out in detail in [4].

\section{Absolute convergence of the product form trees}

The compensation approach constructs the formal solutions $X_{s, t}$ and $x_{s, t}$ of the equilibrium equations. The next step is to prove that the series (25), (26) and (29), which define $X_{s, t}$ and $x_{s, t}$, converge absolutely. We need absolute convergence to guarantee (27) and (28). These series, however, do not necessarily converge absolutely for all $s$ and $t$, but we will prove

\section{THEOREM 1}

(Absolute convergence)

There exists an index $N$ such that:

(i) For all $s, t \geqslant 0$ and $s+t>N$, including $s=N$ and $t=0$, the series (25), (26) and (29), which define the numbers $X_{s, t}$ and $x_{s, t}$, converge absolutely.

(ii)

$$
\sum_{\substack{s \geqslant 0, t \geqslant 0 \\ s+t>N}}\left(\left|X_{s, t}\right|+\left|x_{s, t}\right|\right)<\infty .
$$

\section{Preliminary results for the proof of theorem 1}

To prove that the series (25), (26) and (29) converge absolutely we need information about the asymptotic behaviour of $\alpha_{i}, \beta_{i}, c_{i}$ and $d_{i}$. Below we start 
to prove that $\alpha_{i}$ and $\beta_{i}$ decrease exponentially fast, for which we need the following monotonicity result.

\section{LEMMA 4}

(i) For all $0<\alpha \leqslant \alpha_{0}=\rho^{2}$,

$$
\begin{aligned}
& X_{+}(\alpha)>\alpha>X_{-}(\alpha)>0, \\
& x_{+}(\alpha)>\alpha>x_{-}(\alpha)>0,
\end{aligned}
$$

and $X_{+}(\alpha) / \alpha$ and $x_{+}(\alpha) / \alpha$ are decreasing and $X_{-}(\alpha) / \alpha$ and $x_{-}(\alpha) / \alpha$ are increasing in $\alpha$.

(ii) For all $0<\beta \leqslant \beta_{0}=\rho$,

$$
\begin{aligned}
& Y_{+}(\beta)>\beta>Y_{-}(\beta)>0, \\
& y_{+}(\beta)>\beta>y_{-}(\beta)>0,
\end{aligned}
$$

and $Y_{+}(\beta) / \beta$ and $y_{+}(\beta) / \beta$ are decreasing and $Y_{-}(\beta) / \beta$ and $y_{-}(\beta) / \beta$ are increasing in $\beta$.

Proof

(i) We prove the results for $X_{ \pm}(\alpha)$. The proofs for $x_{ \pm}(\alpha)$ are similar. Since $\alpha \leqslant \alpha_{0}=\rho^{2}$,

$$
\begin{aligned}
(\rho+1)^{2}-\left(2 \rho+\alpha \gamma_{2}\right) \gamma_{1} & \geqslant(\rho+1)^{2}-\left(2 \rho+\rho^{2} \gamma_{2}\right) \gamma_{1} \\
& =\left(1+\rho\left(1-\gamma_{1}\right)\right)^{2}>0
\end{aligned}
$$

so the discriminant of the quadratic form (13) for fixed $\alpha$ is strictly positive and since $\alpha>0$, this implies that $X_{+}(\alpha)$ and $X_{-}(\alpha)$ are two distinct positive roots. Immediately from its definition, it follows that $X_{+}(\alpha) / \alpha$ is decreasing. Further,

$$
\frac{X_{-}(\alpha)}{\alpha}=\frac{\gamma_{1}}{2 \rho+\alpha \gamma_{2}} \frac{\alpha}{X_{+}(\alpha)}=\frac{\gamma_{1}}{\rho+1+\sqrt{(\rho+1)^{2}-\left(2 \rho+\alpha \gamma_{2}\right) \gamma_{1}}}
$$

which is increasing in $\alpha$. The desired inequalities follow from

$$
\begin{aligned}
& \frac{X_{+}(\alpha)}{\alpha} \geqslant \frac{X_{+}\left(\alpha_{0}\right)}{\alpha_{0}}=\frac{1}{\rho}>1, \\
& \frac{X_{-}(\alpha)}{\alpha} \leqslant \frac{X_{-}\left(\alpha_{0}\right)}{\alpha_{0}}=\frac{\gamma_{1}}{2+\rho \gamma_{2}}<1 .
\end{aligned}
$$

(ii) We only prove the results for $Y_{ \pm}(\beta)$. The proofs for $y_{ \pm}(\beta)$ are similar. Since $\beta \leqslant \beta_{0}=\rho$,

$$
\begin{aligned}
\left(2(\rho+1)-\beta \gamma_{2}\right)^{2}-8 \rho \gamma_{1} & \geqslant\left(2(\rho+1)-\rho \gamma_{2}\right)^{2}-8 \rho \gamma_{1} \\
& =\left(2+\rho \gamma_{1}\right)^{2}-8 \rho \gamma_{1}=\left(2-\rho \gamma_{1}\right)^{2}>0,
\end{aligned}
$$


so the discriminant of the quadratic form (13) for fixed $\beta$ is strictly positive and since $\beta>0$ and $2(\rho+1)-\beta \gamma_{2}>0$, this implies that $Y_{+}(\beta)$ and $Y_{-}(\beta)$ are two distinct positive roots. Immediately from its definition, it follows that $Y_{+}(\beta) / \beta$ is decreasing in $\beta$. Further,

$$
\frac{Y_{-}(\beta)}{\beta}=\frac{2 \rho}{\gamma_{1}} \frac{\beta}{Y_{+}(\beta)},
$$

which is increasing in $\beta$. The desired inequalities follow from

$$
\begin{aligned}
& \frac{Y_{+}(\beta)}{\beta} \geqslant \frac{Y_{+}\left(\beta_{0}\right)}{\beta_{0}}=\frac{2}{\gamma_{1}}>1 . \\
& \frac{Y_{-}(\beta)}{\beta} \leqslant \frac{Y_{-}\left(\beta_{0}\right)}{\beta_{0}}=\rho<1 .
\end{aligned}
$$

COROLLARY

For each left branch in the parameter tree,

$$
\alpha_{0} \geqslant \alpha_{i}>\beta_{l(i)}>\alpha_{l(i)}>0,
$$

where the decrease of $\beta_{l(i)}$ and $\alpha_{l(i)}$ is at least

$$
\beta_{l(i)} \leqslant \frac{\gamma_{1}}{2+\rho \gamma_{2}} \alpha_{i}, \quad \alpha_{l(i)} \leqslant \frac{2 \rho}{2+\rho \gamma_{2}} \beta_{l(i)}
$$

and similarly, for each right branch in the parameter tree,

$$
\alpha_{0} \geqslant \alpha_{i}>\beta_{r(i)}>\alpha_{r(i)}>0
$$

where the decrease of $\beta_{r(i)}$ and $\alpha_{r(i)}$ is at least

$$
\beta_{r(i)} \leqslant \frac{\gamma_{2}}{2+\rho \gamma_{1}} \alpha_{i}, \quad \alpha_{r(i)} \leqslant \frac{2 \rho}{2+\rho \gamma_{1}} \beta_{r(i)} .
$$

\section{Proof}

The corollary is proved by induction: we descend the parameter tree by starting in the root. Assume $0<\alpha_{i} \leqslant \alpha_{0}$, which trivially holds for $i=0$. Then by lemma 4(i),

$$
\begin{aligned}
0<\beta_{l(i)}=X_{-}\left(\alpha_{i}\right) & \leqslant \frac{X_{-}\left(\alpha_{0}\right)}{\alpha_{0}} \alpha_{i} \\
& =\frac{\gamma_{1}}{2+\rho \gamma_{2}} \alpha_{i}
\end{aligned}
$$

and $\beta_{l(i)}=X_{-}\left(\alpha_{i}\right) \leqslant X_{-}\left(\alpha_{0}\right)=\beta_{1}$, thus by lemma 4(ii),

$$
\begin{aligned}
\alpha_{l(i)}=Y_{-}\left(\beta_{l(i)}\right) & \leqslant \frac{Y_{-}\left(\beta_{1}\right)}{\beta_{1}} \beta_{l(i)} \\
& =\frac{2 \rho}{2+\rho \gamma_{2}} \beta_{l(i)} .
\end{aligned}
$$

The inequalities for $\beta_{r(i)}$ and $\alpha_{r(i)}$ can be proved similarly. 
The corollary states that $\alpha_{i}$ and $\beta_{i}$ decrease exponentially fast and uniformly in the depth of the parameter tree. For each step downwards in the parameter tree, $\alpha_{i}$ or $\beta_{i}$ decreases with respect to its predecessor at least with a rate which is the maximum of $\gamma_{1} /\left(2+\rho \gamma_{2}\right), 2 \rho /\left(2+\rho \gamma_{2}\right), \gamma_{2} /\left(2+\rho \gamma_{1}\right)$ and $2 \rho /\left(2+\rho \gamma_{1}\right)$. The asymptotic behaviour of $\alpha_{i}$ and $\beta_{i}$ is stated in

LEMMA 5

If the depth of $\alpha_{i}$ in the parameter tree tends to infinity, then

$$
\frac{\beta_{l(i)}}{\alpha_{i}} \rightarrow \frac{1}{A_{2}}, \quad \frac{\beta_{r(i)}}{\alpha_{i}} \rightarrow \frac{1}{a_{2}}
$$

and if the depth of $\beta_{l(i)}$ and $\beta_{r(i)}$ tends to infinity, then

$$
\frac{\alpha_{l(i)}}{\beta_{l(i)}} \rightarrow A_{1}, \quad \frac{\alpha_{r(i)}}{\beta_{r(i)}} \rightarrow a_{1},
$$

where

$$
\begin{array}{ll}
A_{1}=\frac{\rho+1-\sqrt{(\rho+1)^{2}-2 \rho \gamma_{1}}}{\gamma_{1}}, & A_{2}=\frac{\rho+1+\sqrt{(\rho+1)^{2}-2 \rho \gamma_{1}}}{\gamma_{1}}, \\
a_{1}=\frac{\rho+1-\sqrt{(\rho+1)^{2}-2 \rho \gamma_{2}}}{\gamma_{2}}, & a_{2}=\frac{\rho+1+\sqrt{(\rho+1)^{2}-2 \rho \gamma_{2}}}{\gamma_{2}} .
\end{array}
$$

\section{Proof}

We prove the first limit. The other limits are proved similarly. Let the depth of $\alpha_{i}$ in the parameter tree tend to infinity, then, by virtue of the corollary of lemma $4, \alpha_{i} \rightarrow 0$, so

$$
\frac{\beta_{l(i)}}{\alpha_{i}}=\frac{X_{-}\left(\alpha_{i}\right)}{\alpha_{i}} \rightarrow \frac{A_{1} \gamma_{1}}{2 \rho}=\frac{1}{A_{2}}
$$

The asymptotic behaviour of the coefficients $c_{i}$ is stated in

\section{LEMMA 6}

For all $i=1,2, \ldots$, the coefficients $c_{i}$ are strictly positive. If the depth of $\beta_{i}$ in the parameter tree tends to infinity and $i$ runs through $L$, then

$$
c_{i} \rightarrow C \text {, }
$$

and if $i$ runs through $R$, then

$$
c_{i} \rightarrow c
$$

where

$$
C=\frac{1-A_{1}}{A_{2}-1}, \quad c=\frac{1-a_{1}}{a_{2}-1} .
$$


Proof

We prove the first limit. The other one is proved similarly. Since $\beta_{i} \leqslant \beta_{0}$, we obtain by lemma 4 for $i \in L$ that

$$
\begin{aligned}
& \frac{Y_{-}\left(\beta_{i}\right)}{\beta_{i}} \leqslant \frac{Y_{-}\left(\beta_{0}\right)}{\beta_{0}}=\rho<1, \\
& \frac{Y_{+}\left(\beta_{i}\right)}{\beta_{i}} \geqslant \frac{Y_{+}\left(\beta_{0}\right)}{\beta_{0}}=\frac{1}{\rho}>1,
\end{aligned}
$$

which proves that $c_{i}$ is strictly positive. Let the depth of $\beta_{i}$ in the parameter tree tend to infinity and $i$ run through $L$, then by the corollary of lemma $4, \beta_{i} \rightarrow 0$, so

$$
c_{i}=\frac{1-\frac{Y_{-}\left(\beta_{i}\right)}{\beta_{i}}}{\frac{Y_{+}\left(\beta_{i}\right)}{\beta_{i}}-1} \rightarrow \frac{1-A_{1}}{A_{2}-1}=C .
$$

Before stating the asymptotic behaviour of $d_{i}$ we prove that $d_{i}$ is well defined, i.e., the denominator in the definition of $d_{i}$ does not vanish.

\section{LEMMA 7}

For $0<\alpha \leqslant \alpha_{0}$,

$$
\frac{\left(\alpha \gamma_{1}+2 q \rho\right) \alpha}{X_{+}(\alpha)}+\frac{\left(\alpha \gamma_{2}+2(1-q) \rho\right) \alpha}{x_{+}(\alpha)}-2(\rho+1) \alpha
$$

is monotonously decreasing.

\section{Proof}

We show that the derivative is negative for all $0<\alpha \leqslant \alpha_{0}$. Since

$$
\frac{\mathrm{d}}{\mathrm{d} \alpha} \frac{\left(\alpha \gamma_{1}+2 q \rho\right) \alpha}{X_{+}(\alpha)}=\frac{\gamma_{1} \alpha}{X_{+}(\alpha)}+\frac{\left(\alpha \gamma_{1}+2 q \rho\right) \gamma_{2}}{2 \sqrt{(\rho+1)^{2}-\left(2 \rho+\alpha \gamma_{2}\right) \gamma_{1}}}
$$

and using that both terms are increasing in $\alpha$, yields

$$
\begin{aligned}
\frac{\mathrm{d}}{\mathrm{d} \alpha} \frac{\left(\alpha \gamma_{1}+2 q \rho\right) \alpha}{X_{+}(\alpha)} & \leqslant \frac{\gamma_{1} \alpha_{0}}{X_{+}\left(\alpha_{0}\right)}+\frac{\left(\alpha_{0} \gamma_{1}+2 q \rho\right) \gamma_{2}}{2 \sqrt{(\rho+1)^{2}-\left(2 \rho+\alpha_{0} \gamma_{2}\right) \gamma_{1}}} \\
& =\rho \gamma_{1}+\frac{\left(\rho \gamma_{1}+2 q\right) \rho \gamma_{2}}{2\left(1+\rho\left(1-\gamma_{1}\right)\right)} \\
& <\rho \gamma_{1} 3 / 2+q,
\end{aligned}
$$

where the latter inequality follows from the fact that $\rho /\left(1+\rho\left(1-\gamma_{1}\right)\right)$ is increasing in $\rho$. Similarly,

$$
\frac{\mathrm{d}}{\mathrm{d} \alpha} \frac{\left(\alpha \gamma_{1}+2 q \rho\right) \alpha}{x_{+}(\alpha)}<\rho \gamma_{2} 3 / 2+1-q
$$


Hence, the total derivative is less than

$$
\rho \gamma_{1} 3 / 2+q+\rho \gamma_{2} 3 / 2+1-q-2(\rho+1)=\rho-1<0 .
$$

\section{COROLLARY}

For $0<\alpha<\alpha_{0}$,

$$
\frac{\alpha \gamma_{1}+2 q \rho}{X_{+}(\alpha)}+\frac{\alpha \gamma_{2}+2(1-q) \rho}{x_{+}(\alpha)}-2(\rho+1)>0
$$

Proof

Multiplying the left hand side of the above inequality by $\alpha$ and applying lemma 7 yields

$$
\begin{aligned}
& \frac{\left(\alpha \gamma_{1}+2 q \rho\right) \alpha}{X_{+}(\alpha)}+\frac{\left(\alpha \gamma_{2}+2(1-q) \rho\right) \alpha}{x_{+}(\alpha)}-2(\rho+1) \alpha \\
& >\frac{\left(\alpha_{0} \gamma_{1}+2 q \rho\right) \alpha_{0}}{X_{+}\left(\alpha_{0}\right)}+\frac{\left(\alpha_{0} \gamma_{2}+2(1-q) \rho\right) \alpha_{0}}{x_{+}\left(\alpha_{0}\right)}-2(\rho+1) \alpha_{0}=0 .
\end{aligned}
$$

The corollary implies that the denominator in the definition of $d_{l(i)}$ and $d_{r(i)}$ is positive. Together with lemma 4 it follows that the numerator is also positive. Hence, for all $i \geqslant 1$,

$$
\frac{d_{l(i)}}{d_{i}}<0, \quad \frac{d_{r(i)}}{d_{i}}<0
$$

The next lemma describes the asymptotic behaviour of the coefficients $d_{i}$.

LEMMA 8

If the depth of $\alpha_{i}$ in the parameter tree trends to infinity and $i$ runs through $L$, then

$$
\frac{d_{l(i)}}{d_{i}} \rightarrow-D_{l}, \quad \frac{d_{r(i)}}{d_{i}} \rightarrow-D_{r},
$$

and if $i$ runs through $R$, then

$$
\frac{d_{l(i)}}{d_{i}} \rightarrow-d_{l}, \quad \frac{d_{r(i)}}{d_{i}} \rightarrow-d_{r},
$$

where

$$
\begin{aligned}
D_{l} & =\frac{q A_{2}+(1-q) a_{1}}{q A_{1}+(1-q) a_{1}} C, & D_{r} & =\frac{(1-q)\left(A_{2}-A_{1}\right) \gamma_{1}}{\left(q A_{1}+(1-q) a_{1}\right) \gamma_{2}} C, \\
d_{l} & =\frac{q\left(a_{2}-a_{1}\right) \gamma_{2}}{\left(q A_{1}+(1-q) a_{1}\right) \gamma_{1}} c, & d_{r} & =\frac{q A_{1}+(1-q) a_{2}}{q A_{1}+(1-q) a_{1}} c .
\end{aligned}
$$


Proof

We prove the first limit; the others are proved similarly. Multiplying the numerator and denominator in the definition of $d_{l(i)}$ for $i \in L$ by $\alpha_{i}$ and letting the depth of $\alpha_{i}$ in the parameter tree tend to infinity and $i$ run through $L$, so $\alpha_{i} \rightarrow 0$ by the corollary of lemma 4 , we obtain that

$$
\frac{d_{l(i)}}{d_{i}} \rightarrow-\frac{q A_{2}+(1-q) a_{1}}{q A_{1}+(1-q) a_{1}} C \text {. }
$$

Lemmas 5,6 and 8 provide the ingredients to prove that the series (25), (26) and (29) converge absolutely.

\section{Proof of theorem 1}

We can now prove that the series (25), (26) and (29), which define $X_{s, t}$ and $x_{s, t}$, converge absolutely. Consider a fixed $s \geqslant 0$ and $t \geqslant 1$. Since for all $i$ the constants $\alpha_{i}, \beta_{i}$ and $c_{i}$ are positive, it follows that $X_{s, t}$ and $x_{s, t}$ converge absolutely if and only if

$$
\sum_{i=1}^{\infty}\left|d_{i}\right|\left(\alpha_{p(i)}^{s}+c_{i} \alpha_{i}^{s}\right) \beta_{i}^{t}<\infty .
$$

It will be shown below that the terms in this infinite sum converge exponentially fast. First,

the ratio $\frac{\left|d_{l(i)}\right|\left(\alpha_{i}^{s}+c_{l(i)} \alpha_{l(i)}^{s}\right) \beta_{l(i)}^{t}}{\left|d_{i}\right|\left(\alpha_{p(i)}^{s}+c_{i} \alpha_{i}^{s}\right) \beta_{i}^{t}}$

is abbreviated by $\left\{\begin{array}{l}R_{l}(i, s, t) \text { for all } i \in L, \\ r_{l}(i, s, t) \text { for all } i \in R,\end{array}\right.$

and accordingly,

the ratio $\frac{\left|d_{r(i)}\right|\left(\alpha_{i}^{s}+c_{r(i)} \alpha_{r(i)}^{s}\right) \beta_{r(i)}^{t}}{\left|d_{i}\right|\left(\alpha_{p(i)}^{s}+c_{i} \alpha_{i}^{s}\right) \beta_{i}^{t}}$

is abbreviated by $\left\{\begin{array}{l}R_{r}(i, s, t) \text { for all } i \in L, \\ r_{r}(i, s, t) \text { for all } i \in R .\end{array}\right.$

As the depth of the term $\left|d_{i}\right|\left(\alpha_{p(i)}^{s}+c_{i} \alpha_{i}^{s}\right) \beta_{i}^{t}$ in the compensation tree tends to infinity, then the depth of the numbers $\alpha_{p(i)}, \alpha_{i}$ and $\beta_{i}$ in the parameter tree also 
tends to infinity, and thus by lemmas 5,6 and 8 we obtain if $i$ runs through $L$ that

$$
\begin{aligned}
& R_{l}(i, s, t) \rightarrow R_{l}(s, t)=D_{l}\left(\frac{A_{1}}{A_{2}}\right)^{s+t}, \\
& R_{r}(i, s, t) \rightarrow R_{r}(s, t)=D_{r} \frac{1+c\left(a_{1} / a_{2}\right)^{s}}{1+C\left(A_{1} / A_{2}\right)^{s}}\left(\frac{A_{1}}{A_{2}}\right)^{s}\left(\frac{A_{1}}{a_{2}}\right)^{t},
\end{aligned}
$$

and if $i$ runs through $R$,

$$
\begin{aligned}
& r_{l}(i, s, t) \rightarrow r_{l}(s, t)=d_{l} \frac{1+C\left(A_{1} / A_{2}\right)^{s}}{1+c\left(a_{1} / a_{2}\right)^{s}}\left(\frac{a_{1}}{a_{2}}\right)^{s}\left(\frac{a_{1}}{A_{2}}\right)^{t}, \\
& r_{r}(i, s, t) \rightarrow r_{r}(s, t)=d_{r}\left(\frac{a_{1}}{a_{2}}\right)^{s+t} .
\end{aligned}
$$

Hence, in the limit the terms behave geometrically. The problem is to formulate conditions, in terms of these limiting rates, which guarantee the convergence of the infinite sum. For this we need the notion of a positive geometrical binary tree.

\section{DEFINITION 1}

The numbers $n_{1}, n_{2}, n_{3}, \ldots$ form a positive geometrical binary tree if

(i) the numbers $n_{i}$ have a binary tree structure as depicted in fig. 4,

(ii) the initial values $n_{1}$ and $n_{2}$ are positive,

(iii) the geometrical behaviour is determined by the nonnegative matrix $\left(\begin{array}{l}R_{1}, r_{l} \\ R_{r} r_{r}\end{array}\right)$ such that

$$
\begin{array}{lll}
n_{l(i)}=R_{l} n_{i}, & n_{r(i)}=R_{r} n_{i} & \text { if } n_{i} \text { is a left descendant and } \\
n_{l(i)}=r_{l} n_{i}, & n_{r(i)}=r_{r} n_{i} & \text { if } n_{i} \text { is a right descendant. }
\end{array}
$$

Notice that the tree of numbers $n_{1}, n_{2}, n_{3}, \ldots$ is of the same structure as the compensation tree. Let $\sigma(A)$ denote the spectral radius of the matrix $A$, then in

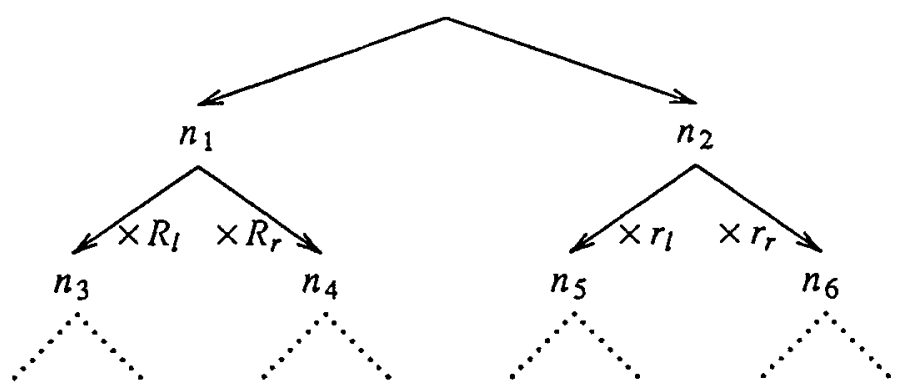

Fig. 4. The binary tree structure of the numbers $n_{i}$. 
particular,

$$
\sigma\left(\begin{array}{ll}
R_{l} & r_{l} \\
R_{r} & r_{r}
\end{array}\right)=\frac{R_{l}+r_{r}+\sqrt{\left(R_{l}-r_{r}\right)^{2}+4 R_{r} r_{l}}}{2}
$$

The next lemma provides a necessary and sufficient condition for the convergence of $\sum_{i=1}^{\infty} n_{i}$.

\section{LEMMA 9}

$\sum_{i=1}^{\infty} n_{i}$ converges if and only if $\sigma\left(\begin{array}{l}R_{i} r_{l} \\ R_{r} r_{r}\end{array}\right)<1$.

\section{Proof}

Define for all $m \geqslant 0$,

$W_{m}=$ the sum of all numbers $n_{i}$ at depth $m$, which are a left descendant,

$w_{m}=$ the sum of all numbers $n_{i}$ at depth $m$, which are a right descendant.

Then for all $m \geqslant 0$,

$$
\left(\begin{array}{c}
W_{m+1} \\
w_{m+1}
\end{array}\right)=\left(\begin{array}{ll}
R_{l} & r_{l} \\
R_{r} & r_{r}
\end{array}\right)\left(\begin{array}{l}
W_{m} \\
w_{m}
\end{array}\right)=\ldots=\left(\begin{array}{ll}
R_{l} & r_{l} \\
R_{r} & r_{r}
\end{array}\right)^{m}\left(\begin{array}{l}
W_{1} \\
w_{1}
\end{array}\right),
$$

where $W_{1}=n_{1}$ and $w_{1}=n_{2}$. Hence

$$
\sum_{i=1}^{\infty} n_{i}=\sum_{m=0}^{\infty}\left(W_{m+1}+w_{m+1}\right)=(1,1) \sum_{m=0}^{\infty}\left(\begin{array}{ll}
R_{l} & r_{l} \\
R_{r} & r_{r}
\end{array}\right)^{m}\left(\begin{array}{l}
W_{1} \\
w_{1}
\end{array}\right)
$$

If $\sigma<1$, then $\left(\begin{array}{c}R_{r} r_{i} \\ R_{r} r_{r}\end{array}\right)^{m}$ converges exponentially fast, so $\sum_{i=1}^{\infty} n_{i}$ converges. If, on the other hand, $\sum_{i=1}^{\infty} n_{i}$ converges, then, since $W_{1}$ and $w_{1}$ are positive and $\left(\begin{array}{l}R_{i} r_{r} \\ R_{r}\end{array} r_{r}\right) \geqslant 0$,

$$
\left(\begin{array}{ll}
R_{l} & r_{l} \\
R_{r} & r_{r}
\end{array}\right)^{m} \rightarrow 0 \quad \text { as } m \rightarrow \infty
$$

which holds if and only if $\sigma<1$.

Hence, the convergence of a positive geometrical binary tree is determined by the spectral radius of the matrix of rates. Since the tree of terms $\left|d_{i}\right|\left(\alpha_{p(i)}^{s}+\right.$ $\left.c_{i} \alpha_{i}^{s}\right) \beta_{i}^{t}$ behaves asymptotically as a positive geometrical binary tree with rates

$$
\left(\begin{array}{ll}
R_{l} & r_{l} \\
R_{r} & r_{r}
\end{array}\right)=\left(\begin{array}{ll}
R_{l}(s, t) & r_{l}(s, t) \\
R_{r}(s, t) & r_{r}(s, t)
\end{array}\right)
$$

we expect that the convergence is also determined by the spectral radius of that matrix. First, let us define 


\section{I.J.B.F. Adan et al. / The shortest queue problem}

DEFINITION 2

For all $n \geqslant 0, \sigma(n)$ is defined by the following equation:

$$
\begin{aligned}
\sigma(n)= & 1 / 2\left(D_{l}\left(A_{1} / A_{2}\right)^{n}+d_{r}\left(a_{1} / a_{2}\right)^{n}\right. \\
& \left.+\sqrt{\left(D_{l}\left(A_{1} / A_{2}\right)^{n}-d_{r}\left(a_{1} / a_{2}\right)^{n}\right)^{2}+4 D_{r} d_{l}\left(A_{1} / A_{2}\right)^{n}\left(a_{1} / a_{2}\right)^{n}}\right) .
\end{aligned}
$$

From this definition we conclude that (cf. (33))

$$
\sigma\left(\begin{array}{ll}
R_{l}(s, t) & r_{l}(s, t) \\
R_{r}(s, t) & r_{r}(s, t)
\end{array}\right)=\sigma(s+t) .
$$

Since $a_{1}$ and $A_{1}$ are positive and less than unity, and $a_{2}$ and $A_{2}$ are larger unity, it follows that the rates $R_{l}(0, n), R_{r}(0, n), r_{l}(0, n)$ and $r_{r}(0, n) \downarrow$ $n \rightarrow \infty$. Hence, $\sigma(n) \downarrow 0$ as $n \rightarrow \infty$ and we can define

\section{DEFINITION 3}

The index $N$ is the smallest index such that $\sigma(N+1)<1$.

Below we prove that if $\sigma(s+t)<1$, or equivalently $s+t>N$, then

$$
\sum_{i=1}^{\infty}\left|d_{i}\right|\left(\alpha_{p(i)}^{s}+c_{i} \alpha_{i}^{s}\right) \beta_{i}^{t}
$$

converges, and otherwise, if $\sigma(s+t)>1$, then it diverges. Assume that $\sigma$ $<1$. Then there exist rates $R_{r}, R_{l}, r_{l}$ and $r_{r}$ such that

$$
\begin{aligned}
& R_{l}>R_{l}(s, t), r_{l}>r_{l}(s, t), \\
& R_{r}>R_{r}(s, t), r_{r}>r_{r}(s, t)
\end{aligned}
$$

and

$$
\sigma\left(\begin{array}{ll}
R_{l} & r_{l} \\
R_{r} & r_{r}
\end{array}\right)<1 .
$$

Then, for all terms $\left|d_{i}\right|\left(\alpha_{p(i)}^{s}+c_{i} \alpha_{i}^{s}\right) \beta_{i}^{t}$ sufficiently deep in the comp tree, at depth at least $M$ say, we obtain for all $i \in L$ that

$$
R_{l}(i, s, t)<R_{l}, \quad R_{r}(i, s, t)<R_{r},
$$

and for all $i \in R$,

$$
r_{l}(i, s, t)<r_{l}, \quad r_{r}(i, s, t)<r_{r} .
$$

Notice that we use here that the ratios converge uniformly in the der compensation tree. Consider the positive geometrical binary tree of $\mathrm{n}$ with rates $\left(\begin{array}{c}R_{t} r_{t} \\ R_{r} r_{r}\end{array}\right)$ and initial values $n_{1}=n_{2}=K$, where $K$ is sufficiently that for all terms $\left|d_{i}\right|\left(\alpha_{p(i)}^{s}+c_{i} \alpha_{i}^{s}\right) \beta_{i}^{t}$ at depth less than or equal to compensation tree, 


\section{I.J.B.F. Adan et al. / The shortest queue problem}

$$
\left|d_{i}\right|\left(\alpha_{p(i)}^{s}+c_{i} \alpha_{i}^{s}\right) \beta_{i}^{t} \leqslant n_{i} .
$$

Im the inequalities (35) it follows that all terms $\left|d_{i}\right|\left(\alpha_{p(i)}^{s}+c_{i} \alpha_{i}^{s}\right) \beta_{i}^{t}$ in the npensation tree are bounded by the numbers $n_{i}$. By lemma 9 , the positive metrical tree of numbers $n_{i}$ is convergent, and thus

$$
\sum_{i=1}^{\infty}\left|d_{i}\right|\left(\alpha_{p(i)}^{s}+c_{i} \alpha_{i}^{s}\right) \beta_{i}^{t} \leqslant \sum_{i=1}^{\infty} n_{i}<\infty .
$$

n the other hand, $\sigma(s+t)>1$, then the tree of terms $\left|d_{i}\right|\left(\alpha_{p(i)}^{s}+c_{i} \alpha_{i}^{s}\right) \beta_{i}^{t}$. can ounded below by a divergent geometrical tree, and thus

$$
\sum_{i=1}^{\infty}\left|d_{i}\right|\left(\alpha_{p(i)}^{s}+c_{i} \alpha_{i}^{s}\right) \beta_{i}^{t}=\infty
$$

se the spectral radius $\sigma(s+t)$ equals unity, nothing can be said in general. ieries (29), which defines $X_{s, 0}$, can be written in the form (32). Accordingly, I be shown for $s$ satisfying $\sigma(s+1)<1$, or equivalently $s+1>N$, that the (32) converges absolutely. This completes the proof of part (i) of theorem 1. mclude this section by proving part (ii) stating that

$$
\sum_{\substack{\geq 0, t \geq 0 \\ i+i>N}}\left(\left|X_{s, t}\right|+\left|x_{s, t}\right|\right)<\infty \text {. }
$$

(9), it follows that

$\sum_{-N}\left|X_{s, 0}\right| 2(\rho+1) \leqslant \sum_{s>N}\left(\left|X_{s-1,1}\right| 2 \rho+\left|X_{s, 1}\right| \gamma_{2}+\left|x_{s-1,1}\right| 2 \rho+\left|x_{s, 1}\right| \gamma_{1}\right)$.

convergence follows once it has been established that

$\sum_{\substack{0, t \geq 1 \\ t i t>N}}\left(\left|X_{s, t}\right|+\left|x_{s, t}\right|\right)<\infty$.

and (26),

$\sum_{\substack{i, t \geq 1 \\ t>N}}\left(\left|X_{s, t}\right|+\left|x_{s, t}\right|\right)$

$$
\begin{aligned}
& \sum_{\substack{s \geqslant 0, t \geqslant 1 \\
s+i>N}} \sum_{i=1}^{\infty}\left|d_{i}\right|\left(\alpha_{p(i)}^{s}+c_{i} \alpha_{i}^{s}\right) \beta_{i}^{t} \\
& \sum_{s=0}^{N-1} \sum_{t=N+1-s}^{\infty} \sum_{i=1}^{\infty}\left|d_{i}\right|\left(\alpha_{p(i)}^{s}+c_{i} \alpha_{i}^{s}\right) \beta_{i}^{t}+\sum_{s=N}^{\infty} \sum_{t=1}^{\infty} \sum_{i=1}^{\infty}\left|d_{i}\right|\left(\alpha_{p(i)}^{s}+c_{i} \alpha_{i}^{s}\right) \beta_{i}^{t} \\
& \sum_{s=0}^{N-1} \sum_{i=1}^{\infty}\left|d_{i}\right|\left(\alpha_{p(i)}^{s}+c_{i} \alpha_{i}^{s}\right) \frac{\beta_{i}^{N+1-s}}{1-\beta_{i}} \\
& +\sum_{i=1}^{\infty}\left|d_{i}\right|\left(\frac{\alpha_{p(i)}^{N}}{1-\alpha_{p(i)}}+c_{i} \frac{\alpha_{i}^{N}}{1-\alpha_{i}}\right) \frac{\beta_{i}}{1-\beta_{i}}<\infty
\end{aligned}
$$


Table 1

The index $N$ for fixed $q=1 / 2$ and increasing values of $\rho$ and $\gamma_{1}$

\begin{tabular}{lll}
\hline$\rho$ & $\gamma_{1}$ & $N$ \\
\hline 0.1 & 0.2 & 1 \\
& 0.5 & 1 \\
0.5 & 0.8 & 0 \\
& 0.2 & 1 \\
& 0.5 & 0 \\
0.9 & 0.8 & 0 \\
& 0.2 & 0 \\
& 0.5 & 0 \\
& 0.8 & 0 \\
\hline
\end{tabular}

since the spectral radius of the matrix of limiting rates for each infinite sum equals $\sigma(N+1)<1$.

\section{Remark 2}

In general, the index $N$ is small. In the special case that $\gamma_{1}=\gamma_{2}$, it follows that $A_{1}=a_{1}$ and $A_{2}=a_{2}$, so $\sigma(n)$ can be simplified as

$$
\sigma(n)=\frac{1-A_{1}}{A_{2}-1}\left(\frac{A_{1}}{A_{2}}\right)^{n-1} \text {. }
$$

Hence, if $\gamma_{1}=\gamma_{2}$, then $\sigma(1)<1$ and thus $N=0$. Only for highly unbalanced systems, that is, as $\gamma_{1} \rightarrow 0$ or $\gamma_{1} \rightarrow 2$, the index $N$ is somewhat larger. In table 1 we list $N$ for fixed $q=1 / 2$ and increasing values of $\rho$ and $\gamma_{1}$.

10. Asymptotic behaviour of $X_{s, t}$ and $x_{s, t}$

It can be easily seen that the constructed solutions $X_{s, t}$ and $x_{s, t}$ are nonnull by investigating the asymptotic behaviour of $X_{s, t}$ and $x_{s, t}$. Below we establish that, for $s+t \rightarrow \infty$,

$$
X_{s, t} \sim X_{s, t}^{1}, \quad x_{s, t} \sim x_{s, t}^{1} .
$$

For $s \geqslant 0, t \geqslant 1$ and $s+t>N$ we have that

$$
X_{s, t}-X_{s, t}^{1}=\sum_{i \in L \backslash\{1\}} d_{i}\left(\alpha_{p(i)}^{s}+c_{i} \alpha_{i}^{s}\right) \beta_{i}^{t} .
$$

By the corollary of lemma $4, \alpha_{i}$ and $\beta_{i}$ decrease along any path of the parameter tree. So $\alpha_{p(i)}$ and $\alpha_{i}$ in (37) are bounded by

$$
\max _{i \geqslant 1} \alpha_{i}=: A<\alpha_{0}
$$

and $\beta_{i}$ by

$$
\max _{i \in L \backslash\{1\}} \beta_{i}=: B<\beta_{1} .
$$


Now let $0 \leqslant u \leqslant s$ and $1 \leqslant v \leqslant t$ and $u+v=N+1$, then

$$
\begin{aligned}
\left|X_{s, t}-X_{s, t}^{1}\right| & \leqslant \sum_{i \in L \backslash\{1\}}\left|d_{i}\right|\left(\alpha_{p(i)}^{s}+c_{i} \alpha_{i}^{s}\right) \beta_{i}^{t} \\
& \leqslant A^{s-u} B^{t-v} \sum_{i \in L \backslash\{1\}}\left|d_{i}\right|\left(\alpha_{p(i)}^{u}+c_{i} \alpha_{i}^{u}\right) \beta_{i}^{v} .
\end{aligned}
$$

Set

$$
M=\max _{\substack{u+v=N+1 \\ u \geqslant 0, v \geqslant 1}} \sum_{i \in L \backslash\{1\}}\left|d_{i}\right|\left(\alpha_{p(i)}^{u}+c_{i} \alpha_{i}^{u}\right) \beta_{i}^{v},
$$

then we obtain for all $s \geqslant 0, t \geqslant 1$ and $s+t>N$ that

$$
\left|X_{s, t}-X_{s, t}^{1}\right| \leqslant M A^{s-N-1} B^{t-N-1},
$$

whence, by using that $A<\alpha_{0}$ and $B<\beta_{1}$, the desired result (36) follows. The asymptotic equivalences for $X_{s, 0}$ and $x_{s, t}$ can be established analogously. It can also be shown that the sequence of solutions $X_{s, t}^{k}$ and $x_{s, t}^{k}$ is an asymptotic expansion of $X_{s, t}$ and $x_{s, t}$ as $s+t \rightarrow \infty$.

\section{Main result}

To find the equilibrium probabilities we developed a compensation approach. The approach constructs the formal solutions $X_{s, t}$ and $x_{s, t}$ of the equilibrium equations by using linear combinations of product forms to satisfy the boundary conditions. The next step was to prove that the series of product forms, which define $X_{s, t}$ and $x_{s, t}$, converge absolutely. These series, however, do not necessarily converge for all $s$ and $t$, but we proved that there exists an index $N$ such that the series for $X_{s, t}$ and $x_{s, t}$ converge absolutely for all $s, t \geqslant 0$ and $s+t>N$, including for $s=N$ and $t=0$, and that for that choice of $N$ the sum of absolute values $\left|X_{s, t}\right|$ and $\left|x_{s, t}\right|$ over all $s, t \geqslant 0$ and $s+t>N$ converges. By finally investigating the asymptotic behaviour of $X_{s, t}$ and $x_{s, t}$ we concluded that the solutions $X_{s, t}$ and $x_{s, t}$ are nonnull. These results are the ingredients to prove our main result, stating that up to a normalizing constant $X_{s, t}$ is $Q_{s, t}$ and $x_{s, t}$ is $q_{s, t}$ for all $s, t \geqslant 0$ and $s+t>N$, including $s=N$ and $t=0$.

\section{THEOREM 2}

(Main result)

For all $s, t \geqslant 0$ and $s+t>N$, including $s=N$ and $t=0$,

$$
Q_{s, t}=C^{-1} X_{s, t}, \quad q_{s, t}=C^{-1} x_{s, t},
$$

where $C$ is the normalizing constant.

To establish the main result, first let

$\mathscr{S}^{(N)}=$ The set of states $(m n)$ with $m>N$ or $n>N$, including state $(m, n)=(N, N)$. 


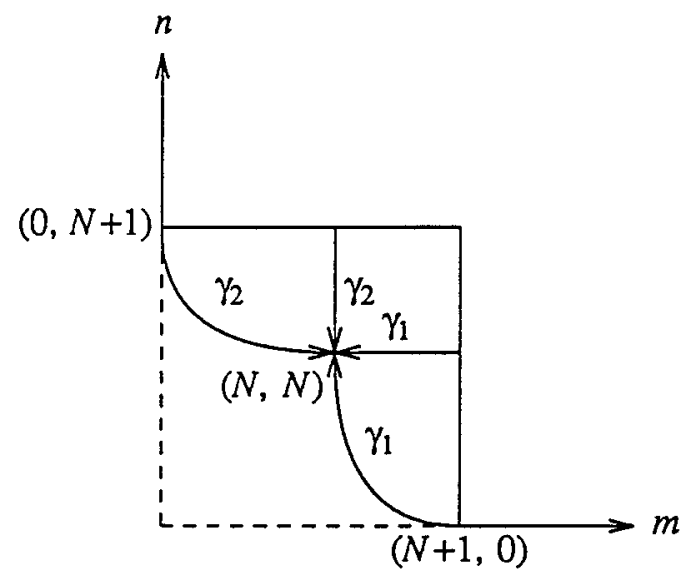

Fig. 5. The transition rates to state $(N, N)$ for the $\mathscr{S}^{(N)}$ restricted process.

$\mathscr{S}^{(N)}$ is the set, in original $(m, n)$-coordinates, on which the series for $X_{s, t}$ and $x_{s, t}$ converge absolutely. Then consider the process restricted to the set $\mathscr{S}^{(N)}$, i.e. the visits of the process in the set $\mathscr{S} \backslash \mathscr{S}^{(N)}$ are not considered. For all states $(m, n),\left(m^{\prime}, n^{\prime}\right) \in \mathscr{S}^{(N)}$, the transition rate of the $\mathscr{S}^{(N)}$ restricted process from $(m, n)$ to $\left(m^{\prime}, n^{\prime}\right)$ is identical to the one of the unrestricted process, except for the transition rates from the states $(k, N+1)$ and $(N+1, k), 0 \leqslant k<N$, to state $(N, N)$. The latter transitions exclusively come from excursions of the unrestricted process in the set $\mathscr{S} \backslash \mathscr{S}^{(N)}$. These excursions always end with a visit at $(N, N)$. That implies that for $0 \leqslant k<N$ the transition rate of the $\mathscr{S}^{(N)}$ restricted process from $(k, N+1)$ to $(N, N)$ is $\gamma_{2}$ and from $(N+1, k)$ to $(N, N)$ is $\gamma_{1}$ (see fig. 5).

Thus the $\mathscr{S}^{(N)}$ restricted process is an irreducible Markov process, whose associated equilibrium equations are identical to the equations of the unrestricted process on the set $\mathscr{S}^{(N)}$, except for the equation in $(N, N)$. Hence, $X_{s, t}$ and $x_{s, t}$ form an absolutely convergent and nonnull solution of all equations associated with the $\mathscr{S}^{(N)}$ restricted process, even the equation in $(N, N)$, for inserting $X_{s, t}$ and $x_{s, t}$ into the other equations on $\mathscr{S}^{(N)}$ and then summing over these equations and changing summations yields the desired equation. Changing summations is allowed by the absolute convergence, stated in theorem 1(ii). By a result of Foster ([12], theorem 1), this proves that the $\mathscr{S}^{(N)}$ restricted process is ergodic. The solutions $X_{s, t}$ and $x_{s, t}$ can be normalized to produce the equilibrium distribution $Q_{s, t}^{(N)}$ and $q_{s, t}^{(N)}$ of the $\mathscr{S}^{(N)}$ restricted process. Since the set $\mathscr{S} \backslash \mathscr{S}^{(N)}$ is finite, it follows that the unrestricted process is also ergodic and the equilibrium probabilities of the unrestricted and $\mathscr{S}^{(N)}$ restricted process are for all $s, t \geqslant 0$ and $s+t>N$, including $s=N$ and $t=0$, related according to

$$
Q_{s, t}=Q_{s, t}^{(N)} P\left(S^{(N)}\right), \quad q_{s, t}=q_{s, t}^{(N)} P\left(S^{(N)}\right),
$$


where $P\left(S^{(N)}\right)$ is the probability that the unrestricted process is in the set $\mathscr{S}^{(N)}$. Since up to a normalizing constant $X_{s, t}$ is $Q_{s, t}^{(N)}$ and $x_{s, t}$ is $q_{s, t}^{(N)}$, that eventually proves theorem 2 .

\section{Product form expression for the normalizing constant}

The series of products for $Q_{s, t}$ and $q_{s, t}$ easily lead to series of products for the normalizing constant $C$ and the moments of the sojourn time. The normalization equation and the expressions for the moments of the sojourn time involve all probabilities $Q_{s, t}$ and $q_{s, t}$, whereas the series for $Q_{s, t}$ and $q_{s, t}$ are only valid for $s+t>N$. Therefore the normalizing constant $C$ and the moments of the sojourn time can only be partly expressed by series of products. In this section we derive a series of products for $C$ (from a balance equation, instead of the normalization equation). The next section is devoted to the moments of the sojourn time.

Let $\rho_{1(2)}$ denote the fraction of time server $1(2)$ is busy, then

$$
\rho_{1}=1-\sum_{t=0}^{\infty} Q_{0, t}, \quad \rho_{2}=1--\sum_{t=0}^{\infty} q_{0, t} .
$$

Equating the number of jobs arriving per unit time and the number of jobs departing per unit time, yields

$$
2 \rho=\rho_{1} \gamma_{1}+\rho_{2} \gamma_{2}
$$

and thus by (38),

$$
2(1-\rho)=\sum_{t=0}^{\infty} Q_{0, t} \gamma_{1}+\sum_{t=0}^{\infty} q_{0, t} \gamma_{2} .
$$

Define for all $s, t \geqslant 0$ the unnormalized quantities $\bar{Q}_{s, t}$ and $\bar{q}_{s, t}$ by

$$
\bar{Q}_{s, t}=C Q_{s, t}, \quad \bar{q}_{s, t}=C q_{s, t}
$$

For all $M \geqslant N$ we obtain by inserting definition (40) into (39) and for $t>M$ the series (25) for $\bar{Q}_{0, t}=X_{0, t}$ and the series (26) for $\bar{q}_{0, t}=x_{0, t}$ that

$$
\begin{aligned}
2(1-\rho) C= & \sum_{t=0}^{M} \bar{Q}_{0, t} \gamma_{1}+\sum_{i=0}^{M} \bar{q}_{0, t} \gamma_{2}+\sum_{t=M+1}^{\infty} \sum_{i \in L} d_{i}\left(1+c_{i}\right) \beta_{i}^{t} \gamma_{1} \\
& +\sum_{t=M+1}^{\infty} \sum_{i \in R} d_{i}\left(1+c_{i}\right) \beta_{i}^{t} \gamma_{2}
\end{aligned}
$$

and thus by changing summations,

$$
\begin{aligned}
2(1-\rho) C= & \sum_{t=0}^{M} \bar{Q}_{0, t} \gamma_{1}+\sum_{t=0}^{M} \bar{q}_{0, t} \gamma_{2}+\sum_{i \in L} d_{i}\left(1+c_{i}\right) \frac{\beta_{i}^{M+1}}{1-\beta_{i}} \gamma_{1} \\
& +\sum_{i \in R} d_{i}\left(1+c_{i}\right) \frac{\beta_{i}^{M+1}}{1-\beta_{i}} \gamma_{2} .
\end{aligned}
$$




\section{The sojourn time}

Below it is indicated how the mean $S$ and the second moment $S^{(2)}$ of the sojourn time can be partly expressed as a series of product forms. First, $S$ and $S^{(2)}$ satisfy

$$
\begin{aligned}
S= & \sum_{s=0}^{\infty} \sum_{t=1}^{\infty}(s+1)\left(Q_{s, t} / \gamma_{1}+q_{s, t} / \gamma_{2}\right)+\sum_{s=0}^{\infty}(s+1) Q_{s, 0}\left(q / \gamma_{2}+(1-q) / \gamma_{1}\right) \\
S^{(2)}= & \sum_{s=0}^{\infty} \sum_{t=1}^{\infty}(2(s+1)+(s+1) s)\left(Q_{s, t} / \gamma_{1}^{2}+q_{s, t} / \gamma_{2}^{2}\right) \\
& +\sum_{s=0}^{\infty}(2(s+1)+(s+1) s) Q_{s, 0}\left(q / \gamma_{2}^{2}+(1-q) / \gamma_{1}^{2}\right)
\end{aligned}
$$

Hence, we need to evaluate series such as, for instance,

$$
\sum_{s=0}^{\infty} \sum_{t=1}^{\infty}(s+1) Q_{s, t}=C^{-1} \sum_{s=0}^{\infty} \sum_{t=1}^{\infty}(s+1) \bar{Q}_{s, t} .
$$

For all $M \geqslant N$ we obtain by inserting for $s+t>M$ the series (25) for $\bar{Q}_{s, t}=X_{s, t}$ that

$$
\begin{aligned}
\sum_{s=0}^{\infty} \sum_{t=1}^{\infty}(s+1) \bar{Q}_{s, t}= & \sum_{s=0}^{M-1} \sum_{t=1}^{M-s}(s+1) \bar{Q}_{s, t} \\
& +\sum_{s=0}^{M-1} \sum_{t=M-s+1}^{\infty}(s+1) \sum_{i \in L} d_{i}\left(\alpha_{p(i)}^{s}+c_{i} \alpha_{i}^{s}\right) \beta_{i}^{t} \\
& +\sum_{s=M}^{\infty} \sum_{t=1}^{\infty}(s+1) \sum_{i \in L} d_{i}\left(\alpha_{p(i)}^{s}+c_{i} \alpha_{i}^{s}\right) \beta_{i}^{t} .
\end{aligned}
$$

Changing summations and inserting the equality

$$
\sum_{s=M}^{\infty}(s+1) \alpha^{s}=\alpha^{M}\left(\frac{1}{(1-\alpha)^{2}}+\frac{M}{1-\alpha}\right),
$$

which holds for $0<\alpha<1$, finally leads to

$$
\begin{aligned}
\sum_{s=0}^{\infty} & \sum_{t=1}^{\infty}(s+1) \bar{Q}_{s, t} \\
= & \sum_{s=0}^{M-1} \sum_{t=1}^{M-s}(s+1) \bar{Q}_{s, t}+\sum_{s=0}^{M-1}(s+1) \sum_{i \in L} d_{i}\left(\alpha_{p(i)}^{s}+c_{i} \alpha_{i}^{s}\right) \frac{\beta_{i}^{M-s+1}}{1-\beta_{i}} \\
& +\sum_{i \in L} d_{i}\left(\frac{\alpha_{p(i)}^{M}\left(1+M\left(1-\alpha_{p(i)}\right)\right)}{\left(1-\alpha_{p(i)}\right)^{2}}+c_{i} \frac{\alpha_{i}^{M}\left(1+M\left(1-\alpha_{i}\right)\right)}{\left(1-\alpha_{i}\right)^{2}}\right) \frac{\beta_{i}}{1-\beta_{i}} .
\end{aligned}
$$


Analogously, product form expressions can be derived for the other series in the expressions for $S$ and $S^{(2)}$, or for other quantities of interest such as higher moments of the sojourn time.

\section{Threshold shortest queue problem}

The compensation approach bears flexibility towards small modifications in the model. In particular, we show that the compensation approach can be used for two related and important problems. In this section we treat the threshold shortest queue problem and in the next section the shortest queue problem for parallel multi server queues.

Let us first explain what we mean by the threshold shortest queue problem. If an arriving job finds $m$ jobs in front of the faster server and $n$ jobs in front of the slower one, then the job joins the queue in front of the faster server if $m<n+T$ and else the other queue. Thus arriving jobs are sent to the faster queue, unless that queue is much longer than the other one. The slower queue now functions as a dynamic overflow queue. The threshold value $T$ can be used as a parameter to balance the utilization of both servers. Suppose that server 1 is the faster one. In fig. 6 we depict the transition rate diagram for the threshold routing problem. The difference with fig. 1 is that the diagonal in fig. 1 is translated to

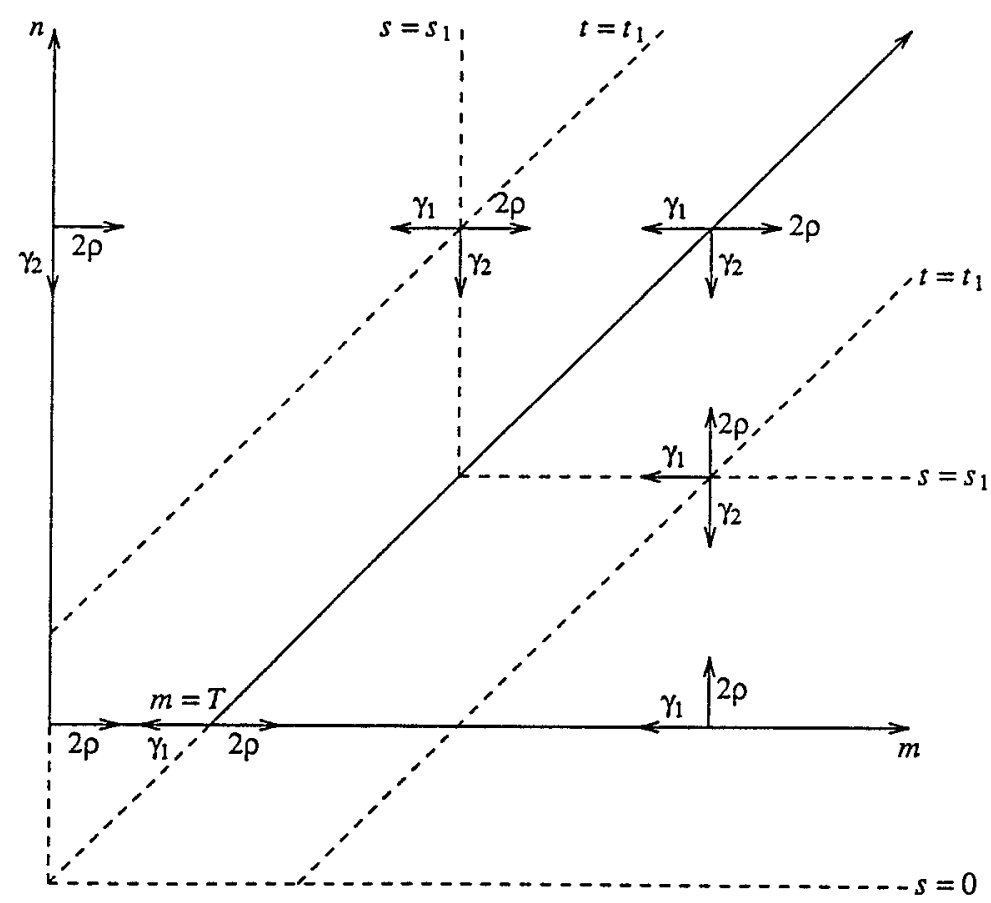

Fig. 6. Transition rate diagram for the threshold routing problem. 
the right. Hence we have to construct solutions on two regions, which are of different shape. The compensation approach for the threshold routing, however, follows the same lines as for the shortest queue routing, except that the compensation on the boundary $s=0$ in the lower triangle now becomes compensation on the boundary $s=T$. The equilibrium equations on $s=T$ state that (cf. eq. (7))

$$
q_{T, t}\left(2 \rho+\gamma_{1}\right)=q_{T, t+1} \gamma_{1}+q_{T+1, t-1} \gamma_{2} \text { if } t>1 \text {. }
$$

Once the equivalent of lemma 2(ii) is established, the rest of the argument to prove the analogy of theorems 1 and 2 is similar. The following lemma summarizes the compensation on the boundary $s=T$ in the lower triangle. The proof is similar to that of lemma 2.

\section{LEMMA 10}

Let for all $s \geqslant T$ and $t \geqslant 1$,

$$
z_{s, t}=k_{1} y_{+}^{s}(\beta) \beta^{t}+k_{2} y_{-}^{s}(\beta) \beta^{t} \text {. }
$$

Then $z_{s, t}$ satisfies eqs. (5) and (43) if $k_{1}$ and $k_{2}$ satisfy

$$
k_{2}=-\frac{y_{+}^{T}(\beta)}{y_{-}^{T}(\beta)} \frac{y_{-}(\beta)-\beta}{y_{+}(\beta)-\beta} k_{1} .
$$

\section{Two multi server queues}

In this section we discuss the extension to parallel multi server queues. The compensation approach for multi server queues follows the same lines as for single server queues, except that the multi server equivalent for the compensation on the boundary $s=0$ is required. Once the multi server equivalent of lemma 2 is established, the rest of the argument to establish the product form representation for the equilibrium probabilities is essentially analogous. Suppose that server 1 is replaced by a multi server queue with $M_{1}$ servers and server 2 by a multi server queue with $M_{2}$ servers. The servers at the first queue work with rate $\gamma_{1} / M_{1}$ and at the second queue with rate $\gamma_{2} / M_{2}$. For $s+t \geqslant M_{2}$ eq. (3) now extends to

$$
\begin{aligned}
& Q_{0, t}\left(2 \rho+\gamma_{2}\right)=Q_{0, t+1} \gamma_{2}+Q_{1, t-1} \gamma_{1} / M_{1}, \\
& Q_{s, t}\left(2 \rho+\gamma_{2}+s \gamma_{1} / M_{1}\right)=Q_{s-1, t+1} 2 \rho+Q_{s, t+1} \gamma_{2}+Q_{s+1, t-1}(s+1) \gamma_{1} / M_{1}, \\
& \quad 0<s<M_{1}-1, \\
& Q_{M_{1}-1, t}\left(2 \rho+\gamma_{2}+\left(M_{1}-1\right) \gamma_{1} / M_{1}\right) \\
& \quad=Q_{M_{1}-2, t+1} 2 \rho+Q_{M_{1}-1, t+1} \gamma_{2}+Q_{M_{1}, t-1} \gamma_{1} .
\end{aligned}
$$

Let $\beta$ be positive and less than unity. Then we show how to compensate for the error of the product $k_{1} Y_{+}^{s}(\beta) \beta^{t}$ on the boundaries $s=0,1, \ldots, M_{1}-1$. For 
the purpose of analysis we rewrite the product $k_{1} Y_{+}^{s}(\beta) \beta^{t}$ as $k_{1} f_{s} \beta^{s+t}$, where for all $s \geqslant M_{1}-1$,

$$
f_{s}=\left(Y_{+}(\beta) / \beta\right)^{s} \text {. }
$$

By lemma 1(i) the product $k_{1} f_{s} \beta^{s+t}$ satisfies eq. (1). Below we first show how to determine $f_{s}$ for $0 \leqslant s<M_{1}-1$ such that eqs. (44) and (45) are satisfied. Next we show how to compensate for the error on the boundary $s=M_{1}-1$. Inserting the product $k_{1} f_{s} \beta^{s+t}$ into (44), (45), (46) and (1) and dividing by $k_{1} \beta^{s+t}$ yields that

$$
\begin{aligned}
& f_{0}\left(2 \rho+\gamma_{2}(1-\beta)\right)=f_{1} \gamma_{1} / M_{1}, \\
& f_{s}\left(2 \rho+\gamma_{2}(1-\beta)+s \gamma_{1} / M_{1}\right)=f_{s-1} 2 \rho+f_{s+1}(s+1) \gamma_{1} / M_{1}, \\
& \quad 0<s<M_{1}-1, \\
& f_{M_{1}-1}\left(2 \rho+\gamma_{2}(1-\beta)+\left(M_{1}-1\right) \gamma_{1} / M_{1}\right)=f_{M_{1}-2} 2 \rho+f_{M_{1}} \gamma_{1}, \\
& f_{s}\left(2 \rho+\gamma_{2}(1-\beta)+\gamma_{1}\right)=f_{s-1} 2 \rho+f_{s+1} \gamma_{1}, \quad s \geqslant M_{1} .
\end{aligned}
$$

By summing over the first $s+1$ equations we reduce eqs. (47) and (48) to the following equivalent set of equations.

$$
\sum_{i=0}^{s} f_{i} \gamma_{2}(1-\beta)+f_{s} 2 \rho=f_{s+1}(s+1) \gamma_{1} / M_{1}, \quad 0 \leqslant s<M_{1}-1 .
$$

The set of equations (51) together with the boundary value

$$
f_{M_{1}-1}=\left(Y_{+}(\beta) / \beta\right)^{M_{1}-1}
$$

recursively define positive $f_{s}$ for $0 \leqslant s<M_{1}-1$. The product $k_{1} f_{s} \beta^{s+t}$ now satisfies (44) and (45). To finally satisfy eq. (46) we add the product $k_{2} g_{s} \beta^{s+\ell}$. By defining for $s \geqslant M_{1}-1$,

$$
g_{s}=\left(Y_{-}(\beta) / \beta\right)^{s},
$$

that product satisfies $(1)$. For $0 \leqslant s<M_{1}-1$ the term $g_{s}$ is recursively defined by eqs. (51) with boundary value

$$
g_{M_{1}-1}=\left(Y_{-}(\beta) / \beta\right)^{M_{1}-1},
$$

so the product $k_{2} g_{s} \beta^{s+t}$ satisfies (44) and (45). By linearity, for any $k_{2}$ the linear combination $k_{1} f_{s} \beta^{s+t}+k_{2} g_{s} \beta^{s+t}$ satisfies eq. (1), (44) and (45). Inserting that linear combination into eq. (46) and dividing by $\beta^{M_{1}-1+t}$ yields the following relation for $k_{2}$,

$$
k_{2}=-\frac{f_{M_{1}-2} 2 \rho+f_{M_{1}} \gamma_{1}-f_{M_{1}-1}\left[2 \rho+\gamma_{2}(1-\beta)+\left(M_{1}-1\right) \gamma_{1} / M_{1}\right]}{g_{M_{1}-2} 2 \rho+g_{M_{1}} \gamma_{1}-g_{M_{1}-1}\left[2 \rho+\gamma_{2}(1-\beta)+\left(M_{1}-1\right) \gamma_{1} / M_{1}\right]} k_{1} .
$$


If $k_{2}$ satisfies relation (52), then the linear combination $k_{1} f_{s} \beta^{s+t}+k_{2} g_{s} \beta^{s+t}$ finally satisfies eq. (46). It remains, however, to verify that the denominator in (52) is nonnull. Suppose to the contrary. Then $g_{s}$ is a positive and convergent solution of eqs. (47), (48), (49) and (50). Summing over all equations leads to

$$
\sum_{i=0}^{\infty} g_{i} \gamma_{2}(1-\beta)=0
$$

which is a contradiction. The next lemma summarizes the compensation on the boundary $s=M_{1}-1$ in the upper triangle. An analogous result can be formulated for the compensation on the boundary $s=M_{2}-1$ in the lower triangle.

LEMMA 11

Let $\beta$ be positive and less than unity and let for all $s \geqslant 0, t \geqslant 1$ and $s+t \geqslant M_{2}$,

$$
Z_{s, t}=k_{1} f_{s} \beta^{s+t}+k_{2} g_{s} \beta^{s+t},
$$

where $f_{s}$ and $g_{s}$ are for $s \geqslant M_{1}-1$ defined as

$$
f_{s}=\left(Y_{+}(\beta) / \beta\right)^{s}, \quad g_{s}=\left(Y_{-}(\beta) / \beta\right)^{s},
$$

and for $0 \leqslant s<M_{1}-1$ as the solution of the set of equations (51) with boundary value $f_{M_{1}-1}=\left(Y_{+}(\beta) / \beta\right)^{M_{1}-1}$ and $g_{M_{1}-1}=\left(Y_{-}(\beta) / \beta\right)^{M_{1}-1}$ respectively. Then $Z_{s, t}$ satisfies eqs. (1), (44), (45) and (46) if $k_{1}$ and $k_{2}$ satisfy relation (52).

\section{The bounding geometrical trees}

We now continue with the numerical analysis of the asymmetric shortest queue problem, in particular by closely investigating the convergence properties of the compensation tree.

The series $X_{s, t}$ and $x_{s, t}$ are obtained by a compensation approach, which is algorithmic in nature. Therefore, a natural question now is how these results are exploited for numerical purposes. In [4] it appeared that indeed the analogous results for the symmetric shortest queue offer an efficient numerical procedure with effective error bounds. The rest of the paper will be focused on the numerical application of the product form representation. It is shown that the compensation tree for $X_{s, t}$ and $x_{s, t}$ can be computed very efficiently with bounds for the error of each partial compensation tree. In section 9 we showed that the compensation tree of terms $\left|d_{i}\right|\left(\alpha_{p(i)}^{s}+c_{i} \alpha_{i}^{s}\right) \beta_{i}^{t}$ behaves asymptotically as a positive geometrical binary tree with nonnegative rates

$$
R(s, t)=\left(\begin{array}{ll}
R_{l}(s, t) & r_{l}(s, t) \\
R_{r}(s, t) & r_{r}(s, t)
\end{array}\right)
$$

Because of the exponential convergence, a few terms often suffice to obtain a very accurate approximation for $X_{s, t}$ and $x_{s, t}$. The question is how accurate is a partial 
compensation tree? Below we derive an upper bound on the contribution of the subtrees below the leaves of each partial compensation tree. That upper bound is obtained by bounding these subtrees by geometrical trees.

In the appendix we define for all $s \geqslant 0, t \geqslant 1$ and all nodes $i \geqslant 1$ in the compensation tree of terms $\left|d_{i}\right|\left(\alpha_{p(i)}^{s}+c_{i} \alpha_{i}^{s}\right) \beta_{i}^{t}$, the nonnegative matrices

$$
B(i, s, t)=\left(\begin{array}{ll}
B_{l}(i, s, t) & b_{l}(i, s, t) \\
B_{r}(i, s, t) & b_{r}(i, s, t)
\end{array}\right)
$$

and prove that $B(i, s, t)$ yields a uniform bound for the rate of convergence of the terms in the subtree below $\left|d_{i}\right|\left(\alpha_{p(i)}^{s}+c_{i} \alpha_{i}^{s}\right) \beta_{i}^{t}$. That is, for all left descendants $\left|d_{j}\right|\left(\alpha_{p(j)}^{s}+c_{j} \alpha_{j}^{s}\right) \beta_{j}^{t}$ in the subtree below $\left|d_{i}\right|\left(\alpha_{p(i)}^{s}+c_{i} \alpha_{i}^{s}\right) \beta_{i}^{t}$, it holds that

$$
R_{l}(j, s, t) \leqslant B_{l}(i, s, t), \quad R_{r}(j, s, t) \leqslant B_{r}(i, s, t),
$$

and for all right descendants,

$$
r_{l}(j, s, t) \leqslant b_{l}(i, s, t), \quad r_{r}(j, s, t) \leqslant b_{r}(i, s, t) .
$$

The following theorem summarizes the bounding properties of the matrices $B(i, s, t)$. That theorem is proved in the appendix.

\section{THEOREM 3}

For all nodes $i$ in the compensation tree of terms $\left|d_{i}\right|\left(\alpha_{p(i)}^{s}+c_{i} \alpha_{i}^{s}\right) \beta_{i}^{t}$, the subtree below node $i$ is bounded by the positive geometrical binary tree with the same initial values and rates $B(i, s, t)$.

Let for all $i \geqslant 1, s \geqslant 0$ and $t \geqslant 1$,

$W(i, s, t)=$ the weight of all left descendants in the subtree below $\left|d_{i}\right|\left(\alpha_{p(i)}^{s}+c_{i} \alpha_{i}^{s}\right) \beta_{i}^{t}$,

$w(i, s, t)=$ the weight of all right descendants in the subtree below $\left|d_{i}\right|\left(\alpha_{p(i)}^{s}+c_{i} \alpha_{i}^{s}\right) \beta_{i}^{t}$.

By theorem 3, the weights $W(i, s, t)$ and $w(i, s, t)$ are bounded by the weight of all left, respectively all right descendants in the positive geometrical tree with the initial values $n_{1}=\left|d_{l(i)}\right|\left(\alpha_{i}^{s}+c_{l(i)} \alpha_{l(i)}^{s}\right) \beta_{l(i)}^{t}$ and $n_{2}=\left|d_{r(i)}\right|\left(\alpha_{i}^{s}+c_{r(i)} \alpha_{r(i)}^{s}\right) \beta_{r(i)}^{t}$ and rates $B(i, s, t)$. From relation (34), these bounds can be calculated easily. This leads to

$$
\left(\begin{array}{c}
W(i, s, t) \\
w(i, s, t)
\end{array}\right) \leqslant \sum_{m=0}^{\infty} B(i, s, t)^{m}\left(\begin{array}{c}
\left|d_{l(i)}\right|\left(\alpha_{i}^{s}+c_{l(i)} \alpha_{l(i)}^{s}\right) \beta_{l(i)}^{t} \\
\left|d_{r(i)}\right|\left(\alpha_{i}^{s}+c_{r(i)} \alpha_{r(i)}^{s}\right) \beta_{r(i)}^{t}
\end{array}\right) .
$$

The upper bound is finite if and only if $\sigma(B(i, s, t))<1$ and in that case the upper bound can be simplified to

THEOREM 4

For all $i \geqslant 1, s \geqslant 0$ and $t \geqslant 1$, if $\sigma(B(i, s, t))<1$, then

$$
\left(\begin{array}{c}
W(i, s, t) \\
w(i, s, t)
\end{array}\right) \leqslant(I-B(i, s, t))^{-1}\left(\begin{array}{c}
\left|d_{l(i)}\right|\left(\alpha_{i}^{s}+c_{l(i)} \alpha_{l(i)}^{s}\right) \beta_{l(i)}^{t} \\
\left|d_{r(i)}\right|\left(\alpha_{i}^{s}+c_{r(i)} \alpha_{r(i)}^{s}\right) \beta_{r(i)}^{t}
\end{array}\right),
$$

where $I$ denotes the identity matrix. 
Assume $\sigma(B(i, s, t))<1$ holds for all nodes $i$ in the compensation tree. Then by theorem 4 we can give an upper bound for the error of each partial compensation tree by computing upper bounds for the weight of the subtrees below the leaves. But how do we know for sure that the condition for the spectral radii holds for all nodes? In the appendix we prove the following monotonicity properties of the matrices $B(i, s, t)$.

\section{LEMMA 12}

(i) $B(i, s, t)$ decreases monotonously along any path in the compensation tree for fixed $s$ and $t$.

(ii) $B(i, s, t) \downarrow R(s, t)$ as the depth of node $i$ in the compensation tree tends to infinity.

(iii) $B(i, s, t)$ decreases monotonously and exponentially fast as $s \rightarrow \infty$ for fixed $i$ and $t$.

(iv) $B(i, s, t)$ decreases monotonously and exponentially fast as $t \rightarrow \infty$ for fixed $i$ and $s$.

Since the inequality $0 \leqslant A \leqslant D$ implies $\sigma(A) \leqslant \sigma(D)$ (see e.g. [13]), it follows by lemma 12(i) that if $\sigma(B(i, s, t))<1$ for $i=1$ and $i=2$, then that inequality holds for all nodes $i$. By lemma 12(iii) and 12(iv) the spectral raddi $\sigma(B(1, s, t))$ and $\sigma(B(2, s, t))$ decrease exponentially fast as $s+t \rightarrow \infty$, so $\sigma(B(1, s, t))$ and $\sigma(B(2, s, t))$ are less than unity for $s+t$ sufficiently large. Now we have all ingredients for the computation of the compensation tree.

\section{The basic scheme for the computation of the compensation tree}

Below we describe the basic scheme for the computation of the series (25) and (26), which define $X_{s, t}$ and $x_{s, t}$ for $s \geqslant 0$ and $t \geqslant 1$, with a relative error of $\varepsilon$. Assume that we can compute bounds right at the beginning of the compensation tree, or equivalently, that both $\sigma(B(1, s, t))$ and $\sigma(B(2, s, t))$ are less than unity. That assumption will be relaxed later on.

\section{Step 0. (Initialization) \\ Compute

$$
\begin{aligned}
& \tilde{X}_{s, t}=d_{1}\left(\alpha_{0}^{s}+c_{1} \alpha_{1}^{s}\right) \beta_{1}^{t}, \\
& \tilde{x}_{s, t}=d_{2}\left(\alpha_{0}^{s}+c_{2} \alpha_{2}^{s}\right) \beta_{2}^{t},
\end{aligned}
$$

and set $k=1$.

Step 1. (Compute all terms at depth $k+1$ in the compensation tree and the bounds) Compute for each term $d_{i}\left(\alpha_{p(i)}^{s}+c_{i} \alpha_{i}^{s}\right) \beta_{i}^{t}$ at depth $k$ in the compensation tree its immediate successors $d_{l(i)}\left(\alpha_{i}^{s}+c_{l(i)} \alpha_{l(i)}^{s}\right) \beta_{l(i)}^{t}$ and $d_{r(i)}\left(\alpha_{i}^{s}+c_{r(i)} \alpha_{r(i)}^{s}\right) \beta_{r(i)}^{t}$ 
and add them to $\tilde{X}_{s, t}$ and $\tilde{x}_{s, t}$ respectively. Compute the upper bounds $U(i, s, t)$ and $u(i, s, t)$ for the weight of all left respectively right descendants in the subtree below $d_{i}\left(\alpha_{p(i)}^{s}+c_{i} \alpha_{i}^{s}\right) \beta_{i}^{t}$, excluding its initial values $d_{l(i)}\left(\alpha_{i}^{s}+\right.$ $\left.c_{l(i)} \alpha_{l(i)}^{s}\right) \beta_{l(i)}^{t}$ and $d_{r(i)}\left(\alpha_{i}^{s}+c_{r(i)} \alpha_{r(i)}^{s}\right) \beta_{r(i)}^{t}$ (because we already added them to $\tilde{X}_{s, t}$ and $\left.\tilde{x}_{s, t}\right)$. That is, by theorem 4 ,

$$
\begin{aligned}
\left(\begin{array}{l}
U(i, s, t) \\
u(i, s, t)
\end{array}\right)= & B(i, s, t)(I-B(i, s, t))^{-1} \\
& \times\left(\begin{array}{c}
\left|d_{l(i)}\right|\left(\alpha_{i}^{s}+c_{l(i)} \alpha_{l(i)}^{s}\right) \beta_{l(i)}^{t} \\
\left|d_{r(i)}\right|\left(\alpha_{i}^{s}+c_{r(i)} \alpha_{r(i)}^{s}\right) \beta_{r(i)}^{t}
\end{array}\right) .
\end{aligned}
$$

Step 2. (Convergence)

Let $I_{k}$ denote the set of indices $i$ of terms $d_{i}\left(\alpha_{p(i)}^{s}+c_{i} \alpha_{i}^{s}\right) \beta_{i}^{t}$, which are at depth $k$ in the compensation tree. If

$$
\sum_{i \in I_{h}} U(i, s, t) \leqslant \varepsilon \times\left\{\tilde{X}_{s, t}-\sum_{i \in I_{h}} U(i, s, t)\right\}
$$

and

$$
\sum_{i \in I_{k}} u(i, s, t) \leqslant \varepsilon \times\left\{\tilde{x}_{s, t}-\sum_{i \in I_{k}} u(i, s, t)\right\},
$$

then the relative accuracy $\varepsilon$ is attained, so stop and approximate $X_{s, t}$ by $\tilde{X}_{s, t}$ and $x_{s, t}$ by $\tilde{x}_{s, t}$; otherwise repeat step 1 with $k=k+1$.

The basic scheme computes the compensation tree with effective error bounds. The bounds are essentialy based on theorem 3. The analogy of theorem 3 also holds for the product form tree in expression (41) for the normalizing constant.

\section{LEMMA 13}

For all nodes $i$ in the tree of terms $\left|d_{i}\right|\left(\alpha_{p(i)}^{s}+c_{i} \alpha_{i}^{s}\right) \beta_{i}^{t} /\left(1-\beta_{i}\right)$, the subtree below node $i$ is bounded by the positive geometrical binary tree with the same initial values and rates $B(i, s, t)$.

Proof

By the corollary of lemma $4, \beta_{l(j)}<\beta_{j}<1$ for all $j \geqslant 1$. Hence, by theorem 3 , we obtain for all left descendants $j$ in the subtree below node $i$, that

$$
\begin{aligned}
\left|d_{l(j)}\right|\left(\alpha_{j}^{s}+c_{l(j)} \alpha_{l(j)}^{s}\right) \frac{\beta_{l(j)}^{t}}{1-\beta_{l(j)}} & \leqslant\left|d_{l(j)}\right|\left(\alpha_{j}^{s}+c_{l(j)} \alpha_{l(j)}^{s}\right) \beta_{l(j)}^{t} \frac{1}{\left(1-\beta_{j}\right)} \\
& \leqslant B_{l}(i, s, t)\left|d_{j}\right|\left(\alpha_{p(j)}^{s}+c_{j} \alpha_{j}^{s}\right) \frac{\beta_{j}^{t}}{\left(1-\beta_{j}\right)} .
\end{aligned}
$$

The other inequalities can be obtained similarly. 
Accordingly, it can be shown that the analogy of theorem 3 holds for the product form trees in the expressions for the moments of the sojourn time (cf. expression (42)). Hence, to compute the product form trees in the expression (41) for the normalizing constant and in the expressions for the moments of the sojourn time, we can use the same scheme as for the computation of the compensation tree.

\section{Remark 3 (convergence test)}

In the convergence test we implicitly use that $X_{s, t}$ and $x_{s, t}$ are positive. In section 10 we proved that $X_{s, t} \sim X_{s, t}^{1}$ and $x_{s, t} \sim x_{s, t}^{1}$ as $s+t \rightarrow \infty$. Hence $X_{s, t}$ and $x_{s, t}$ are positive for $s+t$ sufficiently large. Since $X_{s, t}$ and $x_{s, t}$ are $Q_{s, t}$ and $q_{s, t}$ up to a normalizing constant, and therefore have constant sign, this proves that $X_{s, t}$ and $x_{s, t}$ are positive for all $s+t>N$.

\section{Remark 4 (convergence of the bounds)}

The quality of the computation scheme depends on the rate at which the upper bound

$$
\sum_{i \in I_{k}}\left(\begin{array}{c}
U(i, s, t) \\
u(i, s, t)
\end{array}\right)
$$

converges to 0 as $k \rightarrow \infty$. It follows from lemma 12(ii) that the rate of convergence of the upper bound is determined by the rate at which the weight

$$
\sum_{i \in I_{k}}\left(\begin{array}{l}
\left|d_{l(i)}\right|\left(\alpha_{i}^{s}+c_{l(i)} \alpha_{l(i)}^{s}\right) \beta_{l(i)}^{t} \\
\left|d_{r(i)}\right|\left(\alpha_{i}^{s}+c_{r(i)} \alpha_{r(i)}^{s}\right) \beta_{r(i)}^{t}
\end{array}\right)
$$

converges to 0 as $k \rightarrow \infty$. Since the compensation tree behaves asymptotically as a geometrical tree with rates $R(s, t)$, it can be easily shown that the rate of convergence of the weight mentioned above, and thus of the upper bound, is determined by $\sigma(R(s, t))=\sigma(s+t)$ (cf. the proof of lemma 9). The spectral radius $\sigma(s+t)$ decreases exponentially fast as $s+t \rightarrow \infty$, and therefore the convergence of the upper bound is faster for states further away from the origin. That aspect will be exploited in section 19.

\section{Remark 5 (alternative computation strategy)}

In each cycle the immediate successors of all the leaves of the partial tree are computed. Thus the number of computed terms doubles in each cycle. Fortunately, few cycles usually suffice to obtain an accurate approximation. In section 20 we propose an alternative computation strategy in which a better use is made of the relative importance of the branches of the tree. 
Remark 6 (computation of bounds)

The basic scheme assumes that $\sigma(B(1, s, t))<1$ and $\sigma(B(2, s, t))<1$ in order to compute bounds right at the beginning of the compensation tree. This can be relaxed to computing bounds as soon as $\sigma(B(i, s, t))<1$. If $s+t>N$, the inequality $\sigma(B(i, s, t))<1$ holds for all nodes $i$ sufficiently deep in the compensation tree. This follows from lemma 12(i) and 12(ii) and the observation that by definition $\sigma(R(s, t))=\sigma(s+t)<1$ for all $s+t>N$.

\section{Remark 7 (computation of $X_{s, 0}$ )}

The series for $X_{s, 0}$ are different from the series for $X_{s, t}$ with $t \geqslant 1$. Therefore, the basic scheme cannot be directly applied to compute $X_{s, 0}$. Instead, $X_{s, 0}$ can be obtained from the equilibrium equations on the line $t=0$, stating that

$$
\begin{aligned}
& X_{s, 0} 2(\rho+1)=X_{s-1,1} 2 \rho+X_{s, 1} \gamma_{2}+x_{s-1,1} 2 \rho+x_{s, 1} \gamma_{1}, \quad \text { if } s>0, \\
& X_{0,0} 2 \rho=X_{0,1} \gamma_{2}+x_{0,1} \gamma_{1} .
\end{aligned}
$$

All quantities at the right hand side can be computed by the basic scheme.

\section{Solving the equilibrium equations in a bounded region}

Analogously to (40) first define for all $m$ and $n$ the unnormalized quantities $\bar{p}_{m, n}$ by

$$
\bar{p}_{m, n}=C p_{m, n} .
$$

For all $M \geqslant N$ it holds that the quantities $\bar{p}_{m, n}$ outside the rectangular $\{(m, n)$, $0 \leqslant m, n \leqslant M\}$ can be expressed by the series of products, and therefore can be efficiently computed by the basic scheme. The quantities $\bar{p}_{m, n}$ inside that rectangular can be solved numerically from the equilibrium equations, and are in particular required for the computation of the normalizing constant $C$ and the moments of the sojourn time (cf. sections 12 and 13). Although the equilibrium equations inside the rectangular $\{(m, n), 0 \leqslant m, n \leqslant M\}$ can be solved by standard means, we develop for that purpose a highly efficient and numerically stable algorithm, which is based on the special structure of the shortest queue problem.

Denote by level $l$ the set of states $(m, n)$ for which the longest queue has length $l$. Then at all levels higher than $M(\geqslant N)$, the quantities $\bar{p}_{m, n}$ can be computed by the series of products. Below we show that at the lower levels $\bar{p}_{m, n}$ can be solved efficiently from the equilibrium equations. Based on the special property that at any level the only flow to a higher level is via the state on the diagonal, we reduce the problem of simultaneously solving the equilibrium equations in the rectangular $\{(m, n), 0 \leqslant m, n \leqslant M\}$ to that of recursively 
solving the equations at the levels $M, M-1, \ldots, 1$ and 0 . The equilibrium equations at level $M$ state that

$$
\begin{aligned}
& p_{0, M}\left(2 \rho+\gamma_{2}\right)=p_{1, M} \gamma_{1}+p_{0, M+1} \gamma_{2}, \\
& p_{m, M} 2(\rho+1)=p_{m-1, M} 2 \rho+p_{m+1, M} \gamma_{1}+p_{m, M+1} \gamma_{2}, \quad 0<m<M-1, \\
& p_{M-1, M} 2(\rho+1)=p_{M-2, M} 2 \rho+p_{M, M} \gamma_{1}+p_{M-1, M+1} \gamma_{2}+p_{M-1, M-1} 2 q \rho, \\
& p_{M, 0}\left(2 \rho+\gamma_{1}\right)=p_{M, 1} \gamma_{2}+p_{M+1,0} \gamma_{1}, \\
& p_{M, n} 2(\rho+1)=p_{M, n-1} 2 \rho+p_{M, n+1} \gamma_{2}+p_{M+1, n} \gamma_{1}, \quad 0<n<M-1, \\
& p_{M, M-1} 2(\rho+1)=p_{M, M-2} 2 \rho+p_{M, M} \gamma_{2}+p_{M+1, M-1} \gamma_{1}+p_{M-1, M-1} 2(1-q) \rho .
\end{aligned}
$$

The equation in $(M, M)$ is decomposed into the following two equations. Applying the balance principle

"rate out of the set of states $\{(m, n), 0 \leqslant m, n \leqslant M\}=$ rate into that set of states"

yields

$$
p_{M, M} 2 \rho=\sum_{i=0}^{M} p_{i, M+1} \gamma_{2}+\sum_{i=0}^{M} p_{M+1, i} \gamma_{1}
$$

and to the set of states $\{(m, n), 0 \leqslant m, n \leqslant M\} \backslash\{(M, M)\}$,

$$
p_{M-1, M} 2 \rho+p_{M, M-1} 2 \rho=p_{M, M} 2+\sum_{i=0}^{M-1} p_{i, M+1} \gamma_{2}+\sum_{i=0}^{M-1} p_{M+1, i} \gamma_{1} .
$$

$p_{M-1, M-1}$ is the only probability at a level lower than $M$, occurring in eqs. (53)-(60). Eliminating $p_{M-1, M-1}$ in eqs. (55) and (58), yields a set of linear equations for the probabilities at level $M$. By inserting $\bar{p}_{m, M+1}$ and $\bar{p}_{M+1, n}$ for $m, n=0,1, \ldots, M$ into that set of equations, we can solve the unknown quantities $\bar{p}_{m, n}$ at level $M$. This scheme can be repeated to compute $\bar{p}_{m, n}$ at level $M-1$, $M-2, \ldots, 1,0$. Hence, we reduced the problem of simultaneously solving ( $M+$ $1)^{2}$ equations to that of recursively solving $2 M+1,2 M-1, \ldots$ and 1 equation. The equations for the probabilities at level $M$ form a one dimensional set of inhomogeneous second order recursion relations. We prove that it can be reduced to a first order recursion relation. To formulate that result, we define the sequences $\left\{x_{i}\right\}$ and $\left\{y_{i}\right\}$.

\section{DEFINITION 4}

(i) The sequence $x_{0}, x_{1}, x_{2}, \ldots$ is the solution of

$$
x_{i+1}=x_{i} 2(\rho+1)-x_{i-1} 2 \rho \gamma_{1}, \quad i \geqslant 1
$$

with initial values $x_{0}=1$ and $x_{1}=2 \rho+\gamma_{2}$.

(ii) The sequence $y_{0}, y_{1}, y_{2}, \ldots$ is the solution of

$$
y_{i+1}=y_{i} 2(\rho+1)-y_{i-1} 2 \rho \gamma_{2}, \quad i \geqslant 1,
$$

with initial values $y_{0}=1$ and $y_{1}=2 \rho+\gamma_{1}$. 
THEOREM 5

(i) For $m=M-2, M-3, \ldots, 0$

$$
p_{m, M} x_{m+1}=p_{m+1, M} x_{m} \gamma_{1}+\sum_{i=0}^{m} p_{i, M+1} x_{i}(2 \rho)^{m-i} \gamma_{2}
$$

and the initial value $p_{M-1, M}$ follows from the equation

$$
\begin{aligned}
& p_{M-1, M} 2 \rho\left(x_{M-1} y_{M}+x_{M} y_{M-1}(1-q)\right. \\
& =p_{M, M} 2 x_{M-1}\left(y_{M-1} \gamma_{1}(1-q)+\left(y_{M}-y_{M-1} \gamma_{2} \rho\right) q\right) \\
& \quad+\sum_{i=0}^{M-1} p_{i, M+1}\left(x_{i} y_{M-1}(2 \rho)^{M-i}(1-q) \gamma_{2}+x_{M-1} y_{M} q \gamma_{2}\right) \\
& \quad+\sum_{i=0}^{M-1} p_{M+1, i} x_{M-1}\left(y_{M}-y_{i}(2 \rho)^{M-i}\right) q \gamma_{1} .
\end{aligned}
$$

(ii) For $n=M-2, M-3, \ldots, 0$

$$
p_{M, n} y_{n+1}=p_{M, n+1} y_{n} \gamma_{2}+\sum_{i=0}^{n} p_{M+1, i} y_{i}(2 \rho)^{n-i} \gamma_{1}
$$

and the initial value $p_{M, M-1}$ follows from the equation

$$
\begin{array}{rl}
p_{M, M-1} & 2 \rho\left(y_{M-1} x_{M}+y_{M} x_{M-1} q\right) \\
= & p_{M, M} 2 y_{M-1}\left(x_{M-1} \gamma_{2} q+\left(x_{M}-x_{M-1} \gamma_{1} \rho\right)(1-q)\right) \\
& +\sum_{i=0}^{M-1} p_{M+1, i}\left(y_{i} x_{M-1}(2 \rho)^{M-i} q \gamma_{1}+y_{M-1} x_{M}(1-q) \gamma_{1}\right) \\
& +\sum_{i=0}^{M-1} p_{i, M+1} y_{M-1}\left(x_{M}-x_{i}(2 \rho)^{M-i}\right)(1-q) \gamma_{2}
\end{array}
$$

Proof

We prove eqs. (61) for all $m=0,1, \ldots, M-2$ by induction. For $m=0$ eqs. (61) and (53) are identical. Assume (61) holds for $m=n$. Multiplying (61) for $m=n$ by $2 \rho$ and (54) for $m=n+1$ by $x_{n+1}$ and then adding both equations, yields eq. (61) for $m=n+1$. Hence, eqs. (61) hold for all $m=0,1, \ldots, M-2$. Multiplying (61) for $m=M-2$ by $2 \rho$ and (55) by $x_{M-1}$ and then adding both equations, yields for $m=M-1$ that

$$
\begin{aligned}
p_{M-1, M} x_{M}= & p_{M, M} x_{M-1} \gamma_{1}+\sum_{i=0}^{M-1} p_{i, M+1} x_{i}(2 \rho)^{M-1-i} \gamma_{2} \\
& +p_{M-1, M-1} x_{M-1} 2 q \rho .
\end{aligned}
$$


Similarly, we can prove eq. (63) for all $n=0,1, \ldots, M-2$, and for $n=M-1$ that,

$$
\begin{aligned}
p_{M, M-1} y_{M}= & p_{M, M} y_{M-1} \gamma_{2}+\sum_{i=0}^{M-1} p_{M+1, i} y_{i}(2 \rho)^{M-1-i} \gamma_{1} \\
& +p_{M-1, M-1} y_{M-1} 2(1-q) \rho .
\end{aligned}
$$

Eliminating the probability $p_{M-1, M-1}$ in eqs. (65) and (66) leads to

$$
\begin{aligned}
& p_{M-1, M} x_{M} y_{M-1}(1-q)-p_{M, M-1} x_{M-1} y_{M} q \\
& =p_{M, M} x_{M-1} y_{M-1}\left(\gamma_{1}(1-q)-\gamma_{2} q\right) \\
& \quad+\sum_{i=0}^{M-1} p_{i, M+1} x_{i} y_{M-1}(2 \rho)^{M-1-i} \gamma_{2}(1-q) \\
& \quad+\sum_{i=0}^{M-1} p_{M+1, i} x_{M-1} y_{i}(2 \rho)^{M-1-i} \gamma_{1} q .
\end{aligned}
$$

Together with eq. (60) we have two equations for $p_{M-1, M}$ and $p_{M, M-1}$. Solving both equations yields the desired equations (62) and (64).

Based on theorem 5, the quantities $\bar{p}_{m, n}$ at level $M$ can be efficiently computed. First, $\bar{p}_{M, M}$ follows from eq. (59) by inserting $\bar{p}_{m, M+1}$ and $\bar{p}_{M+1, n}$ for $m, n=0,1, \ldots, M$. Then the initial values $\bar{p}_{M-1, M}$ and $\bar{p}_{M, M-1}$ follow from eqs. (62) and (64) and $\bar{p}_{M-2, M} \rightarrow \bar{p}_{M-3, M} \rightarrow \ldots \rightarrow \bar{p}_{0, M}$ can be successively computed by using (61) and $\bar{p}_{M, M-2} \rightarrow \bar{p}_{M, M-3} \rightarrow \ldots \rightarrow \bar{p}_{M, 0}$ by using (63). To show that the recursion in theorem 5 is numerically stable, we need the following result.

\section{LEMMA 14}

For all $i, j \geqslant 0$

$$
x_{i+j} \geqslant x_{i}(2 \rho)^{j} \geqslant 0, \quad y_{i+j} \geqslant y_{i}(2 \rho)^{j} \geqslant 0 .
$$

\section{Proof}

First, we prove the lemma for fixed $j=1$ and $i \geqslant 0$ by induction. For $i=0$ and $j=1$, inequality (67) trivially holds. Assume (67) holds for $i=k-1$ and $j=1$, then

$$
\begin{aligned}
x_{k+1} & =x_{k} 2(\rho+1)-x_{k-1} 2 \rho \gamma_{1}=x_{k} 2 \rho+x_{k} 2-x_{k-1} 2 \rho \gamma_{1} \\
& \geqslant x_{k} 2 \rho+x_{k-1} 2 \rho\left(2-\gamma_{1}\right) \geqslant x_{k} 2 \rho,
\end{aligned}
$$

which proves (67) for $i=k$ and $j=1$. Now consider an arbitrary $i, j \geqslant 0$. Then, since lemma 14 is already proved for $j=1$ and $i \geqslant 0$,

$$
x_{i+j} \geqslant x_{i+j-1} 2 \rho \geqslant \ldots \geqslant x_{i}(2 \rho)^{j},
$$


which completes the proof of the lemma for $x$. The lemma is proved similarly for $y$.

By lemma 14, it follows that all coefficients in the recursion relations of theorem 5 are nonnegative. Hence, the calculations involve only the addition and multiplication of nonnegative numbers and thus can cause no loss of significant digits. If the series of products for $\bar{p}_{m, n}$ at level $M+1$ are computed with a relative accuracy of $\varepsilon$, then repeated application of the recursion in theorem 5 yields the quantities $\vec{p}_{m, n}$ at lower levels with the same accuracy.

\section{Remark 8 (scaling of the recursion relations)}

By lemma 14, the coefficients $x_{i}$ and $y_{i}$ grow exponentially fast. Therefore, it is numerically sensible to scale the recursion relations in theorem 5. For example, relation (61) can be scaled by dividing both sides by $x_{m+1}$. The resulting recursion relation requires for $i=0,1, \ldots, m$ the calculation of the ratios

$$
\frac{x_{i}(2 \rho)^{-i}}{x_{m+1}(2 \rho)^{-m-1}}
$$

for which an explicit form can be easily derived. By lemma 14, these ratios are bounded by unity.

\section{Remark 9 (alternative numerically stable solution)}

In this section we developed an efficient and numerically stable algorithm, based on the special structure of the problem. To see that it is also possible to use existing, but possibly less efficient algorithms for finding the equilibrium probabilities for finite state Markov chains, notice that up to a normalizing constant, the quantities $\bar{p}_{m, n}$ on the set $\{(m, n), 0 \leqslant m, n \leqslant M\}$ are the equilibrium probabilities of the process restricted to that set. So alternatively, $\bar{p}_{m, n}$ can be solved from the equilibrium equations of that restricted (finite state Markov) process, together with eq. (59) instead of the normalization equation. For solving the equilibrium equations of finite Markov chains, numerically stable algorithms exist, see e.g. Grassmann, Taksar and Heyman [16]. The problem here is that of identifying the rates of the restricted process mentioned above from state $(M, M)$ to the states $(m, M)$ and $(M, n)$ for $m, n=0,1, \ldots, M$ which come from excursions to levels higher than $M$. The rate from $(M, M)$ to $(m, M)$, due to excursions to levels higher than $M$, is given as

$2 \rho\{$ probability that an excursion to levels higher than $M$ ends at $(m, M)\}$, and the rate from $(M, M)$ to $(M ; n)$ as,

$2 \rho\{$ probability that an excursion to levels higher than $M$ ends at $(M, n)\}$. 
The probability that an excursion, starting at $(M, M)$, eventually ends at $(m, M)$ is

$$
\frac{\bar{p}_{m, M+1} \gamma_{2}}{\sum_{i=0}^{M}\left(\bar{p}_{i, M+1} \gamma_{2}+\bar{p}_{M+1, i} \gamma_{1}\right)}
$$

and that an excursion ends at $(M, n)$,

$$
\frac{\bar{p}_{M+1, n} \gamma_{1}}{\sum_{i=0}^{M}\left(\bar{p}_{i, M+1} \gamma_{2}+\bar{p}_{M+1, i} \gamma_{1}\right)}
$$

\section{The combined computation scheme and numerical examples}

We now formulate the computation scheme, which combines the advantages of series of product forms with the recursive algorithm of section 18. First we choose a level $M \geqslant N$. Then the unnormalized quantities $\bar{p}_{m, n}$ at levels higher than $M$ can be computed by the series of products. The quantities $\bar{p}_{m, n}$ at lower levels can be computed by repeated application of the recursion in theorem 5. That recursion initially requires the quantities $\bar{p}_{m, n}$ at level $M+1$, which are computed by the series of products. Finally, the normalizing constant $C$ can be computed from expression (41).

The following observation is important for the choice of $M$. The number of cycles of the basic computation scheme, and thereby the size of the partial compensation tree required to approximate $\bar{p}_{m, n}$ and $\bar{p}_{n, m}$ sufficiently close, depends on the convergence of the upper bounds. It follows from remark 4 that the rate of convergence of the upper bounds is determined by $\sigma(\max (m, n))$, which decreases exponentially fast as $\max (m, n) \rightarrow \infty$. Hence, the rate of convergence is faster for states further away from the origin. This is illustrated in table 2. We list $\bar{p}_{0,1}, \bar{p}_{1,2}$ and $\bar{p}_{2,3}$ and the spectral radii $\sigma(1), \sigma(2)$ and $\sigma(3)$, for

Table 2

Computation of $\bar{p}_{0,1}, \bar{p}_{1,2}$ and $\bar{p}_{2,3}$ by the series of product forms, together with $\sigma(1), \sigma(2), \sigma(3)$ and the depth $D$ required to attain a relative accuracy of $0.1 \%$ for $\gamma_{1}=0.8, q=0.7$ and increasing values of $\rho$

\begin{tabular}{llllllllll}
\hline$\rho$ & $\bar{p}_{0,1}$ & $D$ & $\sigma(1)$ & $\bar{p}_{1,2}$ & $D$ & $\sigma(2)$ & $\bar{p}_{2,3}$ & $D$ & $\sigma(3)$ \\
\hline 0.1 & & & 0.763 & $6.856 \mathrm{e}-6$ & 3 & 0.035 & $6.973 \mathrm{e}-8$ & 2 & 0.002 \\
0.3 & & & 0.529 & 0.001612 & 3 & 0.061 & 0.000151 & 2 & 0.008 \\
0.5 & 0.065715 & 9 & 0.369 & 0.020288 & 3 & 0.058 & 0.005332 & 2 & 0.010 \\
0.7 & 0.178149 & 7 & 0.262 & 0.10758 & 3 & 0.047 & 0.055716 & 2 & 0.009 \\
0.9 & 0.377018 & 6 & 0.190 & 0.37466 & 3 & 0.035 & 0.321458 & 2 & 0.007 \\
\hline
\end{tabular}


Table 3

Computation of $p_{0,0}, p_{1,0}$ and $p_{0,1}$, together with $\sigma(M+1)$ and the depth $D$ required to attain a relative accuracy of $0.1 \%$ for $q=0.7$ and increasing values of $\rho$ and $\gamma_{1}$

\begin{tabular}{lllllllll}
\hline$\rho$ & $\gamma_{1}$ & $p_{0,0}$ & $p_{1,0}$ & $p_{0,1}$ & $M$ & $\sigma(M+1)$ & $D$ & $N$ \\
\hline 0.2 & 0.2 & 0.458891 & 0.326071 & 0.065746 & 2 & 0.077 & 3 & 1 \\
& 0.6 & 0.65048 & 0.153709 & 0.119976 & 1 & 0.082 & 3 & 0 \\
0.6 & 0.9 & 0.667731 & 0.10498 & 0.155919 & 1 & 0.051 & 3 & 0 \\
& 0.2 & 0.053825 & 0.131667 & 0.021253 & 3 & 0.101 & 5 & 1 \\
& 0.6 & 0.201925 & 0.162969 & 0.103235 & 1 & 0.090 & 4 & 0 \\
0.9 & 0.9 & 0.232253 & 0.123756 & 0.152112 & 1 & 0.048 & 3 & 0 \\
& 0.2 & 0.001841 & 0.007135 & 0.001048 & 3 & 0.069 & 5 & 0 \\
& 0.6 & 0.030726 & 0.039101 & 0.022748 & 1 & 0.057 & 4 & 0 \\
0.95 & 0.9 & 0.042493 & 0.035494 & 0.040494 & 1 & 0.032 & 3 & 0 \\
& 0.2 & 0.000539 & 0.002222 & 0.000322 & 3 & 0.060 & 5 & 0 \\
& 0.6 & 0.01381 & 0.018673 & 0.010739 & 1 & 0.052 & 4 & 0 \\
& 0.9 & 0.020045 & 0.017774 & 0.020081 & 1 & 0.030 & 3 & 0 \\
\hline
\end{tabular}

$\gamma_{1}=0.8, q=0.7$ and increasing values of $\rho$. The quantities $\bar{p}_{0,1}, \vec{p}_{1,2}$ and $\bar{p}_{2,3}$ are computed with a relative accuracy of $0.1 \%$. The number $D$ denotes the depth of the computed partial compensation tree, required to attain that accuracy.

Table 2 shows that the spectral radius $\sigma(\max (m, n))$, and thereby the size of the computed partial tree required to approximate $\bar{p}_{m, n}$ sufficiently close, decreases fast for states $(m, n)$ further away from the origin. We conclude that it is numerically sensible to choose the index $M$ such that $\sigma(M+1)$ is sufficiently small. That choice of $M$ might be larger than $N$, but the extra numerical effort to solve the equations in the rectangular $\{(m, n), 0 \leqslant m, n \leqslant M\}$ does not counterbalance the advantages of efficiently computing the series of product forms in states further away from the origin.

In table 3 we list $p_{0,0}, p_{1,0}$ and $p_{0,1}$ with a relative accuracy of $0.1 \%$ for fixed $q=0.7$ and increasing values of $\rho$ and $\gamma_{1}$. The number $D$ denotes the maximal depth of the computed partial trees, required to obtain all quantities $\bar{p}_{m, n}$ at level $M+1$ as well as $C$ with the desired accuracy of $0.1 \%$. We computed all partial trees up to the same depth, so some partial trees might be more accurate than strictly necessary. The examples in table 3 show that the equilibrium probabilities can be computed very efficiently.

We conclude this section by illustrating the effect of the unbalance in service rates on the mean sojourn time $S$ and the coefficient of variation $c v(S)$. In table 4 both $S$ and $c v(S)$ are computed with a relative error of $0.1 \%$. For comparison we computed the mean $S_{c}$ and coefficient of variation $c v\left(S_{c}\right)$ of the sojourn time for a common-queue, but further identical, system. Table 4 illustrates that the unbalance in service rates has little effect on the performance of both systems, 
Table 4

The mean $S$ and coefficient of variation $c v(S)$ of the sojourn time for the parallel-queue system, together with the mean $S_{c}$ and coefficient of variation $c v\left(S_{c}\right)$ of the sojourn time for the "corresponding" common-queue system, for fixed $q=1 / 2$ and increasing values of $\rho$ and $\gamma_{1}$

\begin{tabular}{llllll}
\hline$\rho$ & $\gamma_{1}$ & $S$ & $c v(S)$ & $S_{c}$ & $c v\left(S_{c}\right)$ \\
\hline 0.1 & 0.6 & 1.178 & 1.158 & 1.162 & 1.149 \\
& 0.8 & 1.054 & 1.040 & 1.044 & 1.034 \\
0.3 & 1.0 & 1.018 & 1.000 & 1.010 & 0.995 \\
& 0.6 & 1.294 & 1.168 & 1.203 & 1.102 \\
& 0.8 & 1.178 & 1.037 & 1.123 & 1.000 \\
0.6 & 1.0 & 1.144 & 0.996 & 1.099 & 0.968 \\
& 0.6 & 1.876 & 1.156 & 1.628 & 0.984 \\
& 0.8 & 1.726 & 1.018 & 1.578 & 0.938 \\
0.9 & 1.0 & 1.682 & 0.975 & 1.563 & 0.925 \\
& 0.6 & 5.817 & 1.132 & 5.308 & 0.958 \\
& 0.8 & 5.552 & 1.007 & 5.274 & 0.958 \\
& 1.0 & 5.475 & 0.970 & 5.263 & 0.959 \\
\hline
\end{tabular}

except at light traffic, for then the service time forms the main part of the sojourn time. The parallel-queue system is, of course, more sensitive to unbalance in service rates than the common-queue system.

\section{Alternative computation strategy}

The basic scheme computes in each cycle the immediate successors of all leaves of the current partial tree. For highly unbalanced trees, however, that strategy is inefficient. Trees are highly unbalanced for systems where one server is working much faster than the other one. For example, to approximate $\bar{p}_{3,4}=X_{3,1}$ and $\vec{p}_{4,3}=x_{3,1}$ for $\rho=0.9, \gamma_{1}=0.2$ and $q=0.7$ with an accuracy of $0.1 \%$, the basic scheme computes the compensation tree up to depth 5 . In fig. 7 we depict the relevant part of that partial compensation tree in the sense that the sum of
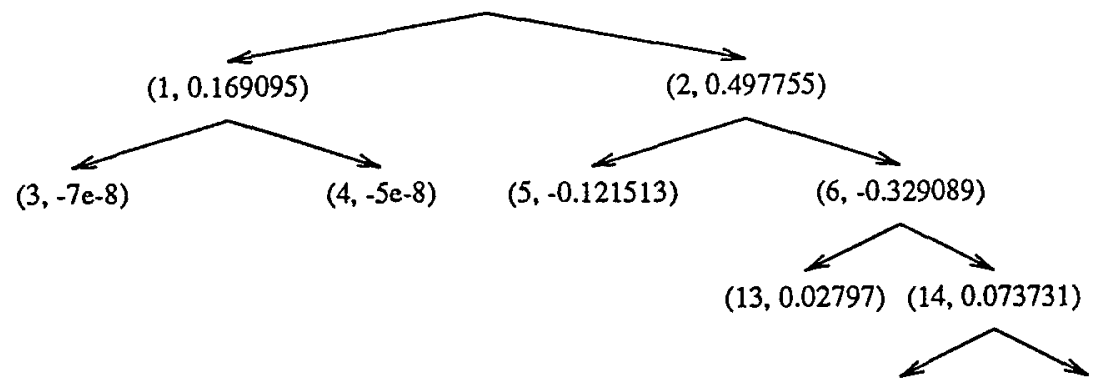

$(29,-0.003619)(30,-0.009453)$

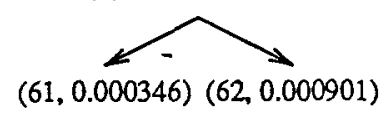

Fig. 7. A part of the compensation tree for $\bar{p}_{3,4}$ and $\bar{p}_{4,3}$ if $\rho=0.9, \gamma_{1}=0.2$ and $q=0.7$. 
absolute values of the other terms is roughly less than $1 e-8$. The pair in each node $i$ stands for $\left(i, d_{i}\left(\alpha_{p(i)}^{3}+c_{i} \alpha_{i}^{3}\right) \beta_{i}\right)$. Figure 7 illustrates that the compensation tree is unbalanced: the weight is concentrated at the very right side of the tree. It is numerically not sensible to compute 62 terms if only 12 terms are relevant. Therefore we propose an alternative strategy, which makes a better use of the relative importance of the branches by computing in each cycle the immediate successors of the leaf the subtree of which has maximum weight, or more precisely, maximum upper bound for its weight. The quantity $U(i, s, t)+u(i, s, t)$ provides an upper bound for the weight of the subtrees below node $l(i)$ and $r(i)$ together, but the strategy mentioned above requires upper bounds for the weight of the subtrees below $l(i)$ and $r(i)$ separately. Therefore we decompose the upper bound $U(i, s, t)$ into the contribution $U_{l}(i, s, t)$ of the subtree below the left descendant $l(i)$, and the contribution $U_{r}(i, s, t)$ of the subtree below the right descendant $r(i)$. Similarly, the upper bound $u(i, s, t)$ is decomposed into the contributions $u_{l}(i, s, t)$ and $u_{r}(i, s, t)$, yielding that

$$
\begin{aligned}
& \left(\begin{array}{l}
U_{l}(i, s, t) \\
u_{l}(i, s, t)
\end{array}\right)=B(i, s, t)(I-B(i, s, t))^{-1}\left(\begin{array}{c}
\left|d_{l(i)}\right|\left(\alpha_{i}^{s}+c_{l(i)} \alpha_{l(i)}^{s}\right) \beta_{l(i)}^{t} \\
0
\end{array}\right) \\
& \left(\begin{array}{l}
U_{r}(i, s, t) \\
u_{r}(i, s, t)
\end{array}\right)=B(i, s, t)(I-B(i, s, t))^{-1}\left(\begin{array}{c}
0 \\
\left|d_{r(i)}\right|\left(\alpha_{i}^{s}+c_{r(i)} \alpha_{r(i)}^{s}\right) \beta_{r(i)}^{t}
\end{array}\right) .
\end{aligned}
$$

Then the weight of the subtree below node $l(i)$ is bounded by $U_{l}(i, s, t)+$ $u_{l}(i, s, t)$ and the total weight of the subtree below node $r(i)$ is bounded by $U_{r}(i, s, t)+u_{r}(i, s, t)$. Based on the decomposed bounds, we can determine in each cycle the leaf the subtree of which has maximum upper bound for its weight. To approximate $\bar{p}_{3,4}$ and $\bar{p}_{4,3}$ for $\rho=0.9, \gamma_{1}=0.2$ and $q=0.7$ with an accuracy of $0.1 \%$, that strategy exactly computes the partial tree depicted in fig. 7 . The difference with the basic scheme is that this time the desired approximations are obtained by computing 12 instead of 62 terms.

\section{Conclusions}

In this paper we studied the asymmetric shortest queue problem and proved that the equilibrium distribution of the lengths of the two queues can be represented by an infinite sum of product form solutions by using a compensation approach. The approach constructs linear combinations of product forms in order to satisfy the boundary conditions. The product form expressions for the equilibrium probabilities easily lead to similar product form expressions for the moments of the sojourn time, or other quantities of interest. These analytical results offer numerically efficient algorithms, mainly due to the exponential convergence of the series of product forms and the tight error bounds. These 
algorithms apply to the exact model. Typically is that on one hand the analysis is rather complicated, whereas on the other hand the resulting procedures are simple, efficient and accurate.

The series of product forms, however, do not necessarily converge absolutely near the origin of the state space. Therefore we proposed a numerically stable and efficient recursive algorithm to solve the equilibrium equations in a bounded region of the state space. This approach can also be used if the convergence of the series of product forms in states around the origin is slow compared to the convergence in states further away from the origin. Combining the advantages of the series of product forms and the recursive algorithm highly reduces the numerical effort to obtain the desired quantities.

Our interest in the shortest queue problem arose out of our work in the design of flexible assembly systems, which can be modeled as queueing models which are related to shortest queue problems. In fact, these queueing models are much more complicated and the present analysis can be viewed as a first attempt to obtain accurate and fast numerical procedures, which are required for the design of flexible assembly systems. An essential characteristic of the queueing models involved is the job-type dependent parallel structure, see e.g. Schwarz [27], Roque [26], Green [17] and Adan, Wessels and Zijm [2]. For a more extensive discussion on the relation between the design of flexible assembly systems and shortest queue problems, the reader is referred to [1]. Future research will be directed to investigating whether the compensation approach can be extended to practically more relevant models. In particular, we mention here the extension to shortest queue problems with a job-type dependent structure and to non-exponential servers, in which case shortest delay routing is more sensible. Another point of interest is the problem of characterizing the class of queueing models having generalized product form solutions.

\section{Appendix}

The appendix contains the proofs of theorem 3 and lemma 12 .

\section{Proof of theorem 3}

For all nodes $i \in L$ in the compensation tree we first derive upper bounds $\bar{R}_{l}(i, s, t)$ and $\bar{R}_{r}(i, s, t)$ for the ratios $R_{l}(i, s, t)$ and $R_{r}(i, s, t)$, so

$$
R_{l}(i, s, t) \leqslant \bar{R}_{l}(i, s, t), \quad R_{r}(i, s, t) \leqslant \bar{R}_{r}(i, s, t),
$$

and accordingly, for all nodes $i \in R$,

$$
r_{l}(i, s, t) \leqslant \bar{r}_{l}(i, s, t), \quad r_{r}(i, s, t) \leqslant \bar{r}_{r}(i, s, t) .
$$


Then we prove that the upper bounds are monotone in the following sense. For all left descendants $j$ in the subtree below node $i$ it holds that,

$$
\bar{R}_{l}(j, s, t) \leqslant \bar{R}_{l}(l(i), s, t), \quad \bar{R}_{r}(j, s, t) \leqslant \bar{R}(l(i), s, t),
$$

and for all right descendants $j$ in the subtree below node $i$,

$$
\bar{r}_{l}(j, s, t) \leqslant \bar{r}_{l}(r(i), s, t), \quad \bar{r}_{r}(j, s, t) \leqslant \bar{r}_{r}(r(i), s, t) .
$$

The proof of theorem 3 is completed by defining $B(i, s, t)$ as

$$
B(i, s, t)=\left(\begin{array}{ll}
B_{l}(i, s, t) & b_{l}(i, s, t) \\
B_{r}(i, s, t) & b_{r}(i, s, t)
\end{array}\right)=\left(\begin{array}{ll}
\bar{R}_{l}(l(i), s, t) & \bar{r}_{l}(r(i), s, t) \\
\bar{R}_{r}(l(i), s, t) & \bar{r}_{r}(r(i), s, t)
\end{array}\right) .
$$

Suppose that $i \in L$. Then we derive an upper bound $\bar{R}_{r}(i, s, t)$ for the ratio $R_{r}(i, s, t)$. The other bounds can be derived similarly. The ratio $R_{r}(i, s, t)$ is given as (see section 9)

$$
R_{r}(i, s, t)=\frac{\left|d_{r(i)}\right|}{\left|d_{i}\right|} \frac{1+c_{r(i)}\left(\frac{\alpha_{r(i)}}{\alpha_{i}}\right)^{s}}{1+c_{i}\left(\frac{\alpha_{i}}{\alpha_{p(i)}}\right)^{2}}\left(\frac{\alpha_{i}}{\alpha_{p(i)}}\right)^{s}\left(\frac{\beta_{r(i)}}{\beta_{i}}\right)^{t}
$$

By substituting the identities $\alpha_{r(i)} / \alpha_{i}=y_{-}\left(\beta_{r(i)}\right) / y_{+}\left(\beta_{r(i)}\right), \alpha_{i} / \alpha_{p(i)}=Y_{-}\left(\beta_{i}\right) /$ $Y_{+}\left(\beta_{i}\right)$ and $\beta_{r(i)} / \beta_{i}=x_{-}\left(\alpha_{i}\right) / X_{+}\left(\alpha_{i}\right)$, we obtain that

$$
R_{r}(i, s, t)=\frac{\left|d_{r(i)}\right|}{\left|d_{i}\right|} \frac{1+c_{r(i)}\left(\frac{y_{-}\left(\beta_{r(i)}\right)}{y_{+}\left(\beta_{r(i)}\right)}\right)^{s}}{1+c_{i}\left(\frac{Y_{-}\left(\beta_{i}\right)}{Y_{+}\left(\beta_{i}\right)}\right)^{s}}\left(\frac{Y_{-}\left(\beta_{i}\right)}{Y_{+}\left(\beta_{i}\right)}\right)^{s}\left(\frac{x_{-}\left(\alpha_{i}\right)}{X_{+}\left(\alpha_{i}\right)}\right)^{t} .
$$

To derive an upper bound for $R_{r}(i, s, t)$, we first need bounds for $c_{j}, c_{r(j)}$ and $\left|d_{r(j)}\right| /\left|d_{j}\right|$.

\section{DEFINITION 5}

For all $0<\alpha<\alpha_{0}$, define

$$
\begin{gathered}
\bar{D}_{l}(\alpha)=\frac{\frac{\left(\alpha \gamma_{1}+2 q \rho\right) \alpha}{X_{-}(\alpha)}+\frac{\left(\alpha \gamma_{2}+2(1-q) \rho\right) \alpha}{x_{+}(\alpha)}}{\frac{\left(\alpha \gamma_{1}+2 q \rho\right) \alpha}{X_{+}(\alpha)}+\frac{\left(\alpha \gamma_{2}+2(1-q) \rho\right) \alpha}{x_{+}(\alpha)}-2(\rho+1) \alpha} \\
\bar{D}_{r}(\alpha)=\frac{\gamma_{1}\left(\alpha \gamma_{2}+2(1-q)\right)\left(A_{2}-A_{1}\right)}{\gamma_{2}\left(\frac{\left(\alpha \gamma_{1}+2 q \rho\right) \alpha}{X_{+}(\alpha)}+\frac{\left(\alpha \gamma_{2}+2(1-q) \rho\right) \alpha}{x_{+}(\alpha)}-2(\rho+1) \alpha\right)},
\end{gathered}
$$




$$
\begin{aligned}
& \bar{d}_{l}(\alpha)=\frac{\gamma_{2}\left(\alpha \gamma_{1}+2 q\right)\left(a_{2}-a_{1}\right)}{\gamma_{1}\left(\frac{\left(\alpha \gamma_{1}+2 q \rho\right) \alpha}{X_{+}(\alpha)}+\frac{\left(\alpha \gamma_{2}+2(1-q) \rho\right) \alpha}{x_{+}(\alpha)}-2(\rho+1) \alpha\right)} \\
& \bar{d}_{r}(\alpha)=\frac{\frac{\left(\alpha \gamma_{1}+2 q \rho\right) \alpha}{X_{+}(\alpha)}+\frac{\left(\alpha \gamma_{2}+2(1-q) \rho\right) \alpha}{x_{-}(\alpha)}}{\frac{\left(\alpha \gamma_{1}+2 q \rho\right) \alpha}{X_{+}(\alpha)}+\frac{\left(\alpha \gamma_{2}+2(1-q) \rho\right) \alpha}{x_{+}(\alpha)}-2(\rho+1) \alpha}
\end{aligned}
$$

and for all $0<\beta<\beta_{0}=\rho$, define

$$
\begin{aligned}
& \underline{C}(\beta)=\frac{1-\frac{Y_{-}(\beta)}{\beta}}{A_{2}-1}, \quad \bar{C}(\beta)=\frac{1-A_{1}}{\frac{Y_{+}(\beta)}{\beta}-1}, \\
& \underline{c}(\beta)=\frac{1-\frac{y_{-}(\beta)}{\beta}}{a_{2}-1}, \quad \bar{c}(\beta)=\frac{1-a_{1}}{\frac{y_{+}(\beta)}{\beta}-1},
\end{aligned}
$$

where

$$
\begin{array}{ll}
A_{1}=\frac{\rho+1-\sqrt{(\rho+1)^{2}-2 \rho \gamma_{1}}}{\gamma_{1}}, & A_{2}=\frac{\rho+1+\sqrt{(\rho+1)^{2}-2 \rho \gamma_{1}}}{\gamma_{1}}, \\
a_{1}=\frac{\rho+1-\sqrt{(\rho+1)^{2}-2 \rho \gamma_{2}}}{\gamma_{2}}, & a_{2}=\frac{\rho+1+\sqrt{(\rho+1)^{2}-2 \rho \gamma_{2}}}{\gamma_{2}} .
\end{array}
$$

\section{LEMMA 15}

For all $i \in L$,

$$
\begin{aligned}
& 0<\underline{C}\left(\beta_{i}\right)<c_{i}<\bar{C}\left(\beta_{i}\right), \\
& \frac{\left|d_{l(i)}\right|}{\left|d_{i}\right|}<\bar{C}\left(\beta_{i}\right) \bar{D}_{l}\left(\alpha_{i}\right), \\
& \frac{\left|d_{r(i)}\right|}{\left|d_{i}\right|}<\bar{C}\left(\beta_{i}\right) \bar{D}_{r}\left(\alpha_{i}\right) ;
\end{aligned}
$$

and for all $i \in R$,

$$
\begin{aligned}
& 0<\underline{c}\left(\beta_{i}\right)<c_{i}<\bar{c}\left(\beta_{i}\right), \\
& \frac{\left|d_{l(i)}\right|}{\left|d_{i}\right|}<\bar{c}\left(\beta_{i}\right) \bar{d}_{l}\left(\alpha_{i}\right), \\
& \frac{\left|d_{r(i)}\right|}{\left|d_{i}\right|}<\bar{c}\left(\beta_{i}\right) \bar{d}_{r}\left(\alpha_{i}\right) .
\end{aligned}
$$


Proof

We proof the bounds for $i \in L$. The proof is similar for $i \in R$. By lemma 4,

$$
\begin{aligned}
& \frac{Y_{+}\left(\beta_{i}\right)}{\beta_{i}}<\lim _{\beta \downarrow 0} \frac{Y_{+}(\beta)}{\beta}=A_{2}, \\
& \frac{Y_{-}\left(\beta_{i}\right)}{\beta_{i}}>\lim _{\beta \downarrow 0} \frac{Y_{-}(\beta)}{\beta}=A_{1} .
\end{aligned}
$$

Inserting these inequalities into the definition of $c_{i}$ yields the bounds for $c_{i}$. Multiplying the denominator and numerator in the definition $d_{l(i)}$ and $d_{r(i)}$ by $\alpha_{i}$, we obtain for $i \in L$ that,

$$
\begin{gathered}
\frac{\left|d_{l(i)}\right|}{\left|d_{i}\right|}=\frac{\frac{\left(\alpha_{i} \gamma_{1}+2 q \rho\right) \alpha_{i}}{X_{-}\left(\alpha_{i}\right)}+\frac{\left(\alpha_{i} \gamma_{2}+2(1-q) \rho\right) \alpha_{i}}{x_{+}\left(\alpha_{i}\right)}-2(\rho+1) \alpha_{i}}{\frac{\left(\alpha_{i} \gamma_{1}+2 q \rho\right) \alpha_{i}}{X_{+}\left(\alpha_{i}\right)}+\frac{\left(\alpha_{i} \gamma_{2}+2(1-q) \rho\right) \alpha_{i}}{x_{+}\left(\alpha_{i}\right)}-2(\rho+1) \alpha_{i}} c_{i} \\
\frac{\left|d_{r(i)}\right|}{\left|d_{i}\right|}=\frac{\gamma_{1}\left(\alpha_{i} \gamma_{2}+2(1-q) \rho\right)\left(\frac{\alpha_{i}}{X_{-}\left(\alpha_{i}\right)}-\frac{\alpha_{i}}{X_{+}\left(\alpha_{i}\right)}\right)}{\gamma_{2}\left(\frac{\left(\alpha_{i} \gamma_{1}+2 q \rho\right) \alpha_{i}}{X_{+}\left(\alpha_{i}\right)}+\frac{\left(\alpha_{i} \gamma_{2}+2(1-q) \rho\right) \alpha_{i}}{x_{+}\left(\alpha_{i}\right)}-2(\rho+1) \alpha_{i}\right)} c_{i} .
\end{gathered}
$$

The bound for $\left|d_{l(i)}\right| /\left|d_{i}\right|$ follows by adding the term $2(\rho+1) \alpha_{i}$ to the numerator and inserting the upper bound for $c_{i}$. The bound for $\left|d_{r(i)}\right| /\left|d_{i}\right|$ follows by inserting the inequalities

$$
\begin{aligned}
& \frac{X_{+}\left(\alpha_{i}\right)}{\alpha_{i}}<\lim _{\alpha \downarrow 0} \frac{X_{+}(\alpha)}{\alpha}=\frac{1}{A_{1}}, \\
& \frac{X_{-}\left(\alpha_{i}\right)}{\alpha_{i}}>\lim _{\alpha \downarrow 0} \frac{X_{-}(\alpha)}{\alpha}=\frac{1}{A_{2}}
\end{aligned}
$$

into the numerator and inserting the upper bound for $c_{i}$.

By lemma 15 we obtain that,

$$
R_{r}(i, s, t)<\bar{C}\left(\beta_{i}\right) \bar{D}_{r}\left(\alpha_{i}\right) \frac{1+\bar{c}\left(\beta_{r(i)}\right)\left(\frac{y_{-}\left(\beta_{r(i)}\right)}{y_{+}\left(\beta_{r(i)}\right)}\right)^{s}}{1+\underline{C}\left(\beta_{i}\right)\left(\frac{Y_{-}\left(\beta_{i}\right)}{Y_{+}\left(\beta_{i}\right)}\right)^{s}}\left(\frac{Y_{-}\left(\beta_{i}\right)}{Y_{+}\left(\beta_{i}\right)}\right)^{s}\left(\frac{x_{-}\left(\alpha_{i}\right)}{X_{+}\left(\alpha_{i}\right)}\right)^{t} .
$$

The right hand side is the desired bound $\bar{R}_{r}(i, s, t)$. 


\section{DEFINITION 6}

For all $s \geqslant 0$ and $t \geqslant 1$ and all $i \in L$, define

$$
\begin{aligned}
& \bar{R}_{l}(i, s, t)=\bar{C}\left(\beta_{i}\right) \bar{D}_{l}\left(\alpha_{i}\right) \frac{1+\bar{C}\left(\beta_{l(i)}\right)\left(\frac{Y_{-}\left(\beta_{l(i)}\right)}{Y_{+}\left(\beta_{l(i)}\right)}\right)^{s}}{1+\underline{C}\left(\beta_{i}\right)\left(\frac{Y_{-}\left(\beta_{i}\right)}{Y_{+}\left(\beta_{i}\right)}\right)^{s}}\left(\frac{Y_{-}\left(\beta_{i}\right)}{Y_{+}\left(\beta_{i}\right)}\right)^{s}\left(\frac{X_{-}\left(\alpha_{i}\right)}{X_{+}\left(\alpha_{i}\right)}\right)^{t}, \\
& \bar{R}_{r}(i, s, t)=\bar{C}\left(\beta_{i}\right) \bar{D}_{r}\left(\alpha_{i}\right) \frac{1+\bar{c}\left(\beta_{r(i)}\right)\left(\frac{y_{-}\left(\beta_{r(i)}\right)}{y_{+}\left(\beta_{r(i)}\right)}\right)^{s}}{1+\underline{C}\left(\beta_{i}\right)\left(\frac{Y_{-}\left(\beta_{i}\right)}{Y_{+}\left(\beta_{i}\right)}\right)^{s}}\left(\frac{Y_{-}\left(\beta_{i}\right)}{Y_{+}\left(\beta_{i}\right)}\right)^{s}\left(\frac{x_{-}\left(\alpha_{i}\right)}{X_{+}\left(\alpha_{i}\right)}\right)^{t}
\end{aligned}
$$

and for all $i \in R$,

$$
\begin{aligned}
& \bar{r}_{l}(i, s, t)=\bar{c}\left(\beta_{i}\right) \bar{d}_{l}\left(\alpha_{i}\right) \frac{1+\bar{C}\left(\beta_{l(i)}\right)\left(\frac{Y_{-}\left(\beta_{l(i)}\right)}{Y_{+}\left(\beta_{l(i)}\right)}\right)^{s}}{1+\underline{c}\left(\beta_{i}\right)\left(\frac{y_{-}\left(\beta_{i}\right)}{y_{+}\left(\beta_{i}\right)}\right)^{s}}\left(\frac{y_{-}\left(\beta_{i}\right)}{y_{+}\left(\beta_{i}\right)}\right)^{s}\left(\frac{X_{-}\left(\alpha_{i}\right)}{x_{+}\left(\alpha_{i}\right)}\right)^{t}, \\
& \bar{r}_{r}(i, s, t)=\bar{c}\left(\beta_{i}\right) \bar{d}_{r}\left(\alpha_{i}\right) \frac{1+\bar{c}\left(\beta_{r(i)}\right)\left(\frac{y_{-}\left(\beta_{r(i)}\right)}{y_{+}\left(\beta_{r(i)}\right)}\right)^{s}}{1+\underline{c}\left(\beta_{i}\right)\left(\frac{y_{-}\left(\beta_{i}\right)}{y_{+}\left(\beta_{i}\right)}\right)^{s}}\left(\frac{y_{-}\left(\beta_{t}\right)}{y_{+}\left(\beta_{i}\right)}\right)^{s}\left(\frac{x_{-}\left(\alpha_{i}\right)}{x_{+}\left(\alpha_{i}\right)}\right)^{t}
\end{aligned}
$$

The following monotonicity properties are required to establish that the upper bounds are monotone.

LEMMA 16

(i) For $0<\alpha<\alpha_{0}$, the functions $\bar{D}_{l}(\alpha), \bar{D}_{r}(\alpha), \bar{d}_{l}(\alpha)$ and $\bar{d}_{r}(\alpha)$ are increasing in $\alpha$.

(ii) For $0<\beta<\beta_{0}=\rho$, the functions $\bar{C}(\beta)$ and $\bar{c}(\beta)$ are increasing in $\beta$ and $C(\beta)$ and $\underline{c}(\beta)$ are decreasing in $\beta$.

(iii) For $0<\alpha<\alpha_{0}$, the ratios $X_{-}(\alpha) / X_{+}(\alpha), x_{-}(\alpha) / X_{+}(\alpha), X_{-}(\alpha) / x_{-}(\alpha)$ and $x_{-}(\alpha) / x_{+}(\alpha)$ are increasing in $\alpha$.

(iv) For $0<\beta<\beta_{0}$, the ratios $Y_{-}(\beta) / Y_{+}(\beta)$ and $y_{-}(\beta) / y_{+}(\beta)$ are increasing in $\beta$.

Proof

(i) We only prove the monotonicity for $\bar{D}_{l}(\alpha)$. The proof is similar for the other ones. By lemma 7, the denominator of $\bar{D}_{l}(\alpha)$ is decreasing in $\alpha$ and positive 
for all $0<\alpha<\alpha_{0}$. By lemma 4 , the numerator is positive and the second term is increasing in $\alpha$. Thus it remains to prove that the first one is also increasing. Since (cf. lemma 7)

$$
\frac{\mathrm{d}}{\mathrm{d} \alpha} \frac{\left(\alpha \gamma_{1}+2 q \rho\right) \alpha}{X_{-}(\alpha)}=\frac{\gamma_{1} \alpha}{X_{-}(\alpha)}-\frac{\left(\alpha \gamma_{1}+2 q \rho\right) \gamma_{2}}{2 \sqrt{(\rho+1)^{2}-\left(2 \rho+\alpha \gamma_{2}\right) \gamma_{1}}}
$$

is decreasing in $\alpha$, we obtain that

$$
\begin{aligned}
\frac{\mathrm{d}}{\mathrm{d} \alpha} \frac{\left(\alpha \gamma_{1}+2 q \rho\right) \alpha}{X_{-}(\alpha)} & >\frac{\gamma_{1} \alpha_{0}}{X_{-}\left(\alpha_{0}\right)}-\frac{\left(\alpha_{0} \gamma_{1}+2 q \rho\right) \gamma_{2}}{2 \sqrt{(\rho+1)^{2}-\left(2 \rho+\alpha_{0} \gamma_{2}\right) \gamma_{1}}} \\
& =2+\rho \gamma_{2}-\frac{\left(\rho \gamma_{1}+2 q\right) \rho \gamma_{2}}{2\left(1+\rho\left(1-\gamma_{1}\right)\right)} \\
& >2+\rho \gamma_{2}-\frac{\left(\rho \gamma_{1}+2 q\right)}{2}>0
\end{aligned}
$$

Hence, the first term in the numerator is increasing in $\alpha$ for $0<\alpha<\alpha_{0}$.

(ii), (iii), (iv) Immediately from lemma 4.

Suppose that node $j$ is a left descendant in the subtree below node $i$ in the compensation tree. Then in the parameter tree, $\alpha_{j}$ is a member of the subtree below $\alpha_{i}$ and thus by the corollary of lemma $4, \alpha_{j}<\alpha_{p(j)} \leqslant \alpha_{i}$. By lemma 4 , it follows that $\beta_{j}=X_{-}\left(\alpha_{p(j)}\right) \leqslant X_{-}\left(\alpha_{i}\right)=\beta_{l(i)}$. So $\alpha_{j}=Y_{-}\left(\beta_{j}\right) \leqslant Y_{-}\left(\beta_{l(i)}\right)=\alpha_{l(i)}$ and $\beta_{r(j)}=x_{-}\left(\alpha_{j}\right) \leqslant x_{-}\left(\alpha_{l(i)}\right)=\beta_{r(l(i))}$. Hence, by lemma 16 ,

$$
\begin{aligned}
\bar{R}_{r}(j, s, t)< & \bar{C}\left(\beta_{l(i)}\right) \bar{D}_{r}\left(\alpha_{l(i)}\right) \frac{1+\bar{c}\left(\beta_{r(l(i))}\right)\left(\frac{y_{-}\left(\beta_{r(l i))}\right)}{y_{+}\left(\beta_{r(l(i))}\right)}\right)^{s}}{1+\underline{C}\left(\beta_{l(i)}\right)\left(\frac{Y_{-}\left(\beta_{j}\right)}{Y_{+}\left(\beta_{j}\right)}\right)^{s}} \\
& \times\left(\frac{Y_{-}\left(\beta_{j}\right)}{Y_{+}\left(\beta_{j}\right)}\right)^{s}\left(\frac{x_{-}\left(\alpha_{l(i)}\right)}{X_{+}\left(\alpha_{l(i)}\right)}\right)^{t},
\end{aligned}
$$

and then using that the function $x^{s} /\left(1+c x^{s}\right)$ is increasing in $x$ for all $x, c \geqslant 0$, finally yields

$$
\bar{R}_{r}(j, s, t) \leqslant \bar{R}_{r}(l(i), s, t) .
$$

Since the other inequalities can be proved similarly, this completes the proof of theorem 3. 
Remark 8 (improvement of the bounds)

The bounds derived in lemma 15 are quite rude. One can easily obtain refinements. For example, the numerator of $\bar{D}_{l}(\alpha)$ can be refined to (cf. the proof of lemma 16(i))

$$
\frac{\left(\alpha \gamma_{1}+2 q \rho\right) \alpha}{X_{-}(\alpha)}+\frac{\left(\alpha \gamma_{2}+2(1-q) \rho\right) \alpha}{x_{+}(\alpha)}-\left(2+\rho \gamma_{2}-\frac{\left(\rho \gamma_{1}+2 q\right)}{2}\right) \alpha,
$$

which is montonously increasing in $\alpha$. More important is that we did not use the property that the terms are alternating in the depth of the compensation tree (see for example fig. 6). This property suggests that the error of each partial tree can be bounded by the absolute weight of its leaves. This is true if the absolute weight of all terms at depth $k$ in the compensation tree decreases monotonously in $k$. Asymptotically, this is indeed the case and numerical experiments revealed that the monotonicity usually holds from the beginning of the compensation tree. The problem is to formulate conditions which guarantee the monotonicity from the beginning or from a certain depth in the compensation tree. For the symmetric problem, the compensation tree of product forms reduces to a linear series and in [4] we showed that the successive terms are alternating and monotonously decreasing in modulus. Thus for the symmetric problem the error of each partial sum can indeed be bounded by the last term.

\section{Proof of lemma 12}

(i) Immediately from the definition of $B(i, s, t)$ and the monotonicity (68).

(ii) We prove that $B_{l}(i, s, t)=\bar{R}_{l}(l(i), s, t) \rightarrow R_{l}(s, t)$ as the depth of node $i$ in the compensation tree tends to infinity. The other limits are proved similarly. It suffices to prove that the bounds for $c_{l(i)}, c_{l(l(i))}$ and $\left|d_{l(l i))}\right| /\left|d_{l(i)}\right|$ are asymptotically tight. As the depth of node $i$ in the compensation tree tends to infinity, then the depth of $\beta_{l(i)}$ in the parameter tree also tends to infinity, so $\beta_{l(i)} \rightarrow 0$ by the corollary of lemma 4 . Since $Y_{-}(\beta) / \beta \rightarrow A_{1}$ and $Y_{+}(\beta) / \beta \rightarrow A_{2}$ as $\beta \rightarrow 0$, we have, as $\beta_{l(i)} \rightarrow 0$, (cf. lemma 6) that

$$
\underline{C}\left(\beta_{l(i)}\right) \text { and } \bar{C}\left(\beta_{l(i)}\right) \rightarrow \frac{1-A_{1}}{A_{2}-1}=C .
$$

Analogously, as the depth of node $i$ in the compensation tree tends to infinity, it can be proved that $\bar{C}\left(\beta_{l(l(i))}\right) \rightarrow C$ and $\bar{C}\left(\beta_{l(i)}\right) \bar{D}_{l}\left(\alpha_{l(i)}\right) \rightarrow D_{l}$.

(iii), (iv) The ratios $Y_{-}\left(\beta_{l(i)}\right) / Y_{+}\left(\beta_{l(i)}\right), x_{-}\left(\alpha_{l(i)}\right) / X_{+}\left(\alpha_{l(i)}\right), \ldots$ are all positive and strictly less than unity. For example, by lemma 4 and its corollary,

$$
0<\frac{x_{-}\left(\alpha_{l(i)}\right)}{X_{+}\left(\alpha_{l(i)}\right)}<\frac{x_{-}\left(\alpha_{0}\right)}{X_{+}\left(\alpha_{0}\right)}=\frac{\rho \gamma_{2}}{2+\rho \gamma_{1}}<1 .
$$

Hence, $B(i, s, t)$ decreases monotonously and exponentially fast as $s \rightarrow \infty$ for fixed $t$ and as $t \rightarrow \infty$ for fixed $s$. 


\section{References}

[1] I.J.B.F. Adan, J. Wessels and W.H.M. Zijm, Flexible assembly and shortest queue problems, in: Proc. Int. Conf. on Modern Production Concepts, Hagen, 1990 (Springer, Berlin) to appear.

[2] I.J.B.F. Adan, J. Wessels and W.H.M. Zijm, Queueing analysis in a flexible assembly system with a job-dependent parallel structure, in: Operations Research Proceedings 1988 (Springer, Berlin, 1989) pp. 551-558.

[3] I.J.B.F. Adan, J. Wessels and W.H.M. Zijm, An asymmetric shortest queue problem, in: Operations Research Proceedings 1989 (Springer, Berlin, 1990) pp. 434-441.

[4] I.J.B.F. Adan, J. Wessels and W.H.M. Zijm, Analysis of the symmetric shortest queue problem, Stochastic Models 6 (1990) 691-713.

[5] J.P.C. Blanc, On a numerical method for calculating state probabilities for queueing systems with more than one waiting line, J. Comput. Appl. Math. 20 (1987) 119-125.

[6] J.P.C. Blanc, The power-series algorithm applied to the shortest-queue model, Memorandum 379, Tilburg University, Department of Economics (1989).

[7] J.W. Cohen and O.J. Boxma, Boundary Value Problems in Queueing System Analysis (NorthHolland, Amsterdam, 1983).

[8] B.W. Conolly, The autostrada queueing problem, J. Appl. Prob. 21 (1984) 394-403.

[9] G. Fayolle, Méthodes analytiques pour les files d'attente couplées, Thesis, Univ. de Paris VI, Paris (1979).

[10] L. Flatto and H.P. McKean, Two queues in parallel, Comm. Pure Appl. Math. 30 (1977) 255-263.

[11] G.J. Foschini and J. Salz, A basic dynamic routing problem and diffusion, IEEE Trans. Commun COM-26 (1978) 320-327.

[12] F.G. Foster, On the stochastic matrices associated with certain queueing processes, Ann. Math. Stat. 24 (1953) 355-360.

[13] F.R. Gantmacher, The Theory of Matrices, vol. 2 (translated by K.A. Hirsch) (Celsea, New York, 1959).

[14] I. Gertsbakh, The shorter queue problem: A numerical study using the matrix-geometric solution, Eur. J. Oper. Res. 15 (1984) 374-381.

[15] W.K. Grassmann, Transient and steady state results for two parallel queues, OMEGA Int. J. Mgmt Sci. 8 (1980) 105-112.

[16] W.K. Grassmann, M.I. Taksar and D.P. Heyman, Regenerative analysis and steady state distributions for Markov chains, Oper. Res. 33 (1985) 1107-1116.

[17] L. Green, A queueing system with general-use and limited-use servers, Oper. Res. 33 (1985) $168-182$.

[18] F.A. Haight, Two queues in parallel, Biometrica 45 (1958) 401-410.

[19] S. Halfin, The shortest queue problem, J. Appl. Prob. 22 (1985) 865-878.

[20] G. Hooghiemstra, M. Keane and S. Van De Ree, Power series for stationary distributions of coupled processor models, SIAM J. Appl. Math. 48 (1988) 1159-1166.

[21] R. Iasnogorodski, Problèmes-frontières dans les files d'attente, Thesis, Univ. de Paris VI, Paris (1979).

[22] J.F.C. Kingman, Two similar queues in parallel, Ann. Math. Statist. 32 (1961) 1314-1323.

[23] C. Knessl, B.J. Matkowsky, Z. Schuss and C. Tier, Two parallel queues with dynamic routing, IEEE Trans. Commun. COM-34 (1986) 1170-1175.

[24] M.F. Neuts, Matrix-Geometric Solutions in Stochastic Models (Johns Hopkins University Press, Baltimore, 1981).

[25] B.M. Rao and M.J.M. Posner, Algorithmic and approximation analysis of the shorter queue model, Naval Res. Log. 34 (1987) 381-398.

[26] D.R. Roque, A note on "Queueing models with lane selection", Oper. Res. 28 (1980) 419-420.

[27] B.L. Schwartz, Queueing models with lane selection: a new class of problems, Oper. Res. 22 (1974) 331-339. 